\title{
WestVirginiaUniversity
}

THE RESEARCH REPOSITORY @ WVU

Graduate Theses, Dissertations, and Problem Reports

2020

\section{Numerical Analysis and Gravity}

Tyler D. Knowles

West Virginia University, tk0014@mix.wvu.edu

Follow this and additional works at: https://researchrepository.wvu.edu/etd

Part of the Numerical Analysis and Computation Commons

\section{Recommended Citation}

Knowles, Tyler D., "Numerical Analysis and Gravity" (2020). Graduate Theses, Dissertations, and Problem Reports. 7560.

https://researchrepository.wvu.edu/etd/7560

This Dissertation is protected by copyright and/or related rights. It has been brought to you by the The Research Repository @ WVU with permission from the rights-holder(s). You are free to use this Dissertation in any way that is permitted by the copyright and related rights legislation that applies to your use. For other uses you must obtain permission from the rights-holder(s) directly, unless additional rights are indicated by a Creative Commons license in the record and/ or on the work itself. This Dissertation has been accepted for inclusion in WVU Graduate Theses, Dissertations, and Problem Reports collection by an authorized administrator of The Research Repository @ WVU.

For more information, please contact researchrepository@mail.wvu.edu. 
Numerical Analysis and Gravity

Tyler D. Knowles, A.B., M.S.

Dissertation submitted

to the Eberly College of Arts and Sciences

at West Virginia University

in partial fulfillment of the requirements for the degree of

Doctor of Philosophy in

Mathematics

Zachariah B. Etienne, Ph.D., Chair

Adam Halasz, Ph.D., Co-Chair

Sean T. McWilliams, Ph.D.

Harvey Diamond, Ph.D.

Casian Pantea, Ph.D.

Department of Mathematics

Morgantown, West Virginia

2020

Keywords: numerical analysis, general relativity, gravitational waves, accelerometry

Copyright 2020 Tyler D. Knowles 


\section{ABSTRACT \\ Numerical Analysis and Gravity}

\section{Tyler D. Knowles}

In this dissertation we apply techniques of numerical analysis to current questions related to understanding gravity. The first question is that of sources of gravitational waves: how can we accurately determine the intrinsic physical parameters of a binary system whose late inspiral and merger was detected by the Laser Interferometer Gravitational-Wave Observatory. In particular, state-of-the-art algorithms for producing theoretical waveforms are as many as three orders of magnitude too slow for timely analysis. We show that direct software optimization produces a two order of magnitude speedup. We also describe documentation efforts undertaken so that the software may be rewritten to enhance both performance and physical realism.

The second question is that of measuring Newton's gravitational constant $G$. In particular, the results of experiments measuring $G$ have differed by as many as ten standard deviations. Measuring the oscillation frequency of a magnetically-levitated microsphere shows promise for sharpening the value of $G$, and the system for this measurement was found to accurately measure low-frequency accelerations. As such, this system forms a prototype for a room-temperature, low-mass accelerometer. At the center of the accelerometer and $G$ measurements lies a new image analysis technique we developed for determining the position of the microsphere to $1.6 \mathrm{~nm}$. 


\section{Table of Contents}

Page

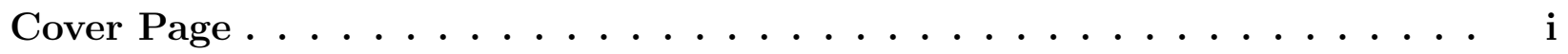

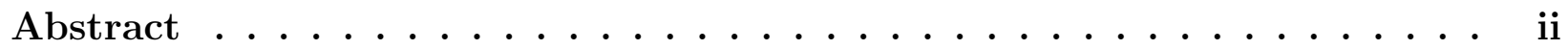

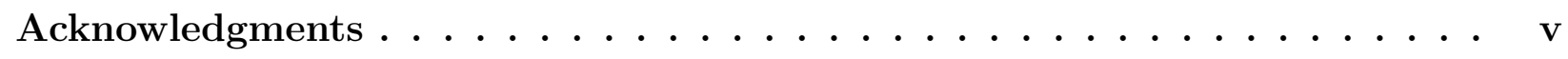

Chapter 1: Introduction and Motivation . . . . . . . . . . . . 1

1.1 Mechanics and Gravity: Basics of General Relativity . . . . . . . . . . . . . 1

1.2 Detecting Gravitational Waves . . . . . . . . . . . . . . . . . . . . . 3

1.3 The Way(ve) of the Future . . . . . . . . . . . . . . . . . . . . . . . . . . 6

1.4 Uncertainty in $G \ldots \ldots \ldots \ldots$

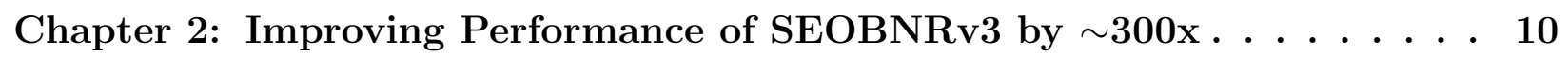

2.1 Abstract . . . . . . . . . . . . . . . . . . . . 10

2.2 Introduction . . . . . . . . . . . . . . . . . . . . . . . . 11

2.3 v3_opt: Optimizations migrated from v2_opt . . . . . . . . . . . . . . 15

2.3 .1 Migrated Optimizations . . . . . . . . . . . . . . . . 15

2.3.2 Guided Automatic Differentiation: A more efficient way of generating symbolic derivatives of the Hamiltonian. . . . . . . . . . . . . . . . . 16

2.3.3 Dense Output: An efficient way of interpolating sparsely-sampled data 20

2.4 Results . . . . . . . . . . . . . . . . . . . . . . . . . . 21

2.4 .1 Determining Faithfulness . . . . . . . . . . . . . . . . . . . 21

2.4 .2 Performance Benchmarks . . . . . . . . . . . . . . . . . . . . . . 24

2.5 Conclusions and Future Work . . . . . . . . . . . . . . . . . . . . . . . . . 26

Chapter 3: Documenting SEOBNRv3 for future approximant optimizations 28

Chapter 4: High Sensitivity accelerometry with a feedback-cooled magnetically levitated microsphere . . . . . . . . . . . . . 34 
4.1 Abstract . . . . . . . . . . . . . . . . . . . . . . . . . . . . . . . . 34

4.2 Introduction $\ldots \ldots \ldots \ldots \ldots$

4.3 Experimental Setup . . . . . . . . . . . . . . . . . . . . . . . . . . 37

$4.3 .1 \quad$ Loading and Trapping of Microspheres $\ldots \ldots \ldots \ldots$

4.3 .2 Table Stabilization . . . . . . . . . . . . . . . . . . . . . . . . 39

$4.3 .3 \quad$ Real-Time Image Analysis and Feedback Cooling _. . . . . . . . . . 39

4.4 Offline Image Analysis $\ldots \ldots \ldots$

4.5 Acceleration Measurement . . . . . . . . . . . . . . . . . . . . 43

4.5 .1 Results . . . . . . . . . . . . . . . . . . . . . . . . . . . . . . . . 45

$4.5 .2 \quad$ Noise Analysis . . . . . . . . . . . . . . . . . . . . . . . . . . 48

4.6 Discussion $\ldots \ldots \ldots \ldots \ldots$

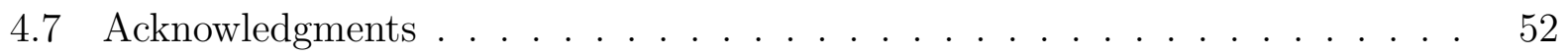

Chapter 5: Active Optical Table Tilt Stabilization $\ldots \ldots \ldots \ldots$

5.1 Abstract . . . . . . . . . . . . . . . . . . . . . . . 53

5.2 Results . . . . . . . . . . . . . . . . . . . . 53

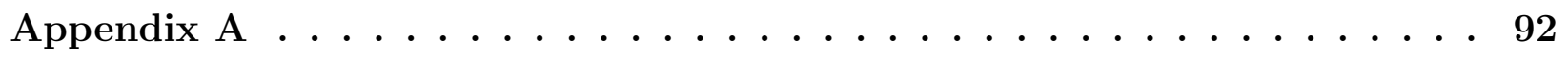

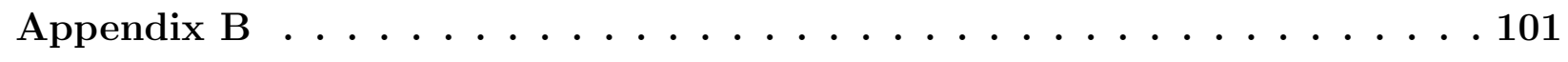




\section{Acknowledgements}

First and foremost I would like to extend my sincerest gratitude to my advisor, Dr. Zachariah B. Etienne, for his patient guidance since I began the Ph.D. program four years ago. His consistent insistence on careful analysis and deep understanding make him an incomparable role model for research and teaching. I am also grateful to my entire dissertation committee for their feedback on this dissertation. A special acknowledgement is due to Dr. Sean T. McWilliams, who acted in an advising capacity for the duration of my program. His breadth of knowledge provides constant perspective on the importance of these research activities. I am also deeply indebted to Dr. Brian D'Urso and Charles W. Lewandowski of the Montana State University Department of Physics for their patience and insight during months of painstaking collaboration.

I am thankful for my Christian mentors who have walked with me through the Ph.D. program: Corey Friend, Lance Rhodes, and Jeff Mattern. Their wisdom and prayers have consistently kept my focus on the Gospel, enabling me to walk worthy of my calling.

Finally, I extend deep gratitude to my parents and siblings for their constant encouragement. I would not have succeeded in graduate school without them. 


\section{Chapter 1}

\section{Introduction and Motivation}

\subsection{Mechanics and Gravity: Basics of General Rela- tivity}

A foundational notion in physics is that of an inertial frame, wherein objects free from external forces travel in straight lines at constant speeds. One advantage to defining and solving physical problems in inertial frames is that physical laws are invariant in inertial frames. That is, a physical experiment conducted on, e.g., a ship sailing in a straight line at constant speed in still waters, will yield the same results as a similar experiment conducted in a train car running on straight tracks at constant speed. This somewhat intuitive notion is integral to classical relativity and was known to Galileo.

One of the first hints that classical relativity may not always hold came from James Clerk Maxwell's laws of electromagnetism, codified in the second half of the 19th century. Maxwell predicted that the speed of light in a vacuum takes the same value in all inertial frames. This is contrary to human intuition and contradicts the notion of relative velocity in classical mechanics. Early in the following century, Albert Einstein postulated his Theory of Special Relativity, which combines both the idea of inertial frames and Maxwell's prediction about the speed of light.

Consider the following thought experiment, illustrated in Figure 1.1. Place a light source on the platform of a train car and a mirror above the source. This light-mirror system 


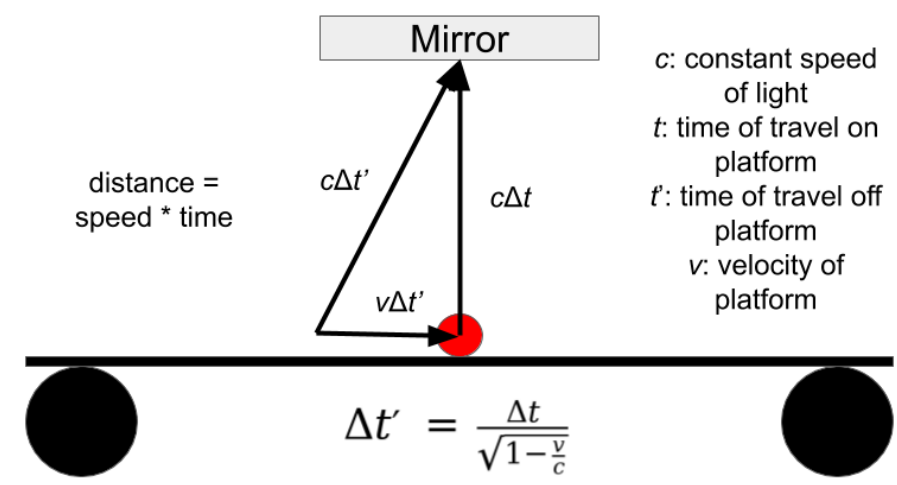

Figure 1.1: Illustration of a thought experiment showing the unintuitive nature of special relativity.

will form a type of clock, one "tick" of which is the time it takes a pulse of light to travel from the source to the mirror. Suppose the car is traveling on a straight section of track at constant velocity $v$. An observer on the platform sees the pulse travel in a straight line up to the mirror. In contrast, an observer near the tracks sees the pulse travel diagonally as the car passes; the relation between the time calculated by the two observers is found easily using the Pythagorean theorem. The result: moving clocks appear to tick more slowly than stationary clocks.

The Theory of Special Relativity does not include a description of gravity. In moving from Special Relativity to his Theory of General Relativity, Einstein assumed the equivalence principle: that inertial mass and gravitational mass are equivalent. Although scientists assumed (and showed experimentally to the best of their ability) the equivalence of inertial and gravitational mass long before Einstein, it was Einstein who was able to mathematically codify the following idea: if gravitational mass and inertial mass are equivalent, then all objects moving in (only) a gravitational field are accelerated equally. This seems, at first, quite strange: how can what we perceive to be empty space uniquely determine an object's trajectory?

This leads to what is called the geodesic hypothesis: an object subject only to gravity follows an extremal (or "shortest") path in spacetime. This description of gravity implies that gravity itself is not a Newtonian force but rather the curvature of spacetime. As summed 
up by Misner, Thorne, and Wheeler: "Space tells matter how to move, and matter tells space how to curve" [1]. Einstein's famous equation of General Relativity is

$$
G^{\alpha \beta}=\frac{8 \pi G}{c^{4}} T^{\alpha \beta}
$$

where $G^{\alpha \beta}$ is the Einstein tensor, describing the curvature of spacetime, and $T^{\alpha \beta}$ is the stress-energy tensor, describing a distribution of energy and momentum. Einstein's General Relativity makes several amazing predictions. Among them is a prediction from the first known closed form solution to Einstein's equation, discovered by Karl Schwarzschild in 1916. This solution describes the geometry of spacetime near a non-rotating spherical point mass $M$, where infinitesimal displacement vectors take the form

$$
\mathrm{d} s^{2}=-\left(1-\frac{2 G M}{r}\right) \mathrm{d} t^{2}+\left(1-\frac{2 G M}{r}\right)^{-1} \mathrm{~d} r^{2}+r^{2} \mathrm{~d} \theta^{2}+r^{2} \sin ^{2} \theta \mathrm{d} \phi^{2}
$$

in spherical coordinates and where $G$ is Newton's gravitational constant. Note in particular the coordinate singularity at $r=2 G M$. When the surface radius of an object is less than $2 G M$ we call the object a black hole. General relativity predicts that black holes exist!

Another prediction of General Relativity is that binary systems (in which two point masses orbit one another) lose energy: the orbital radius of the binary decreases over time and the two objects eventually collide. Energy is radiated away from the binary system in the form of gravitational waves: compressions and stretches (often referred to as "ripples") of spacetime traveling away from the binary and encoding information about how the binary is deforming spacetime in its vicinity (an artist's rendition is given in Figure 1.2). This prediction is confirmed beautifully by the Hulse-Taylor pulsar, whose orbital period can be measured very accurately and whose decrease in periastron time fits the prediction of general relativity very well (see Figure 1.3 and [2]).

\subsection{Detecting Gravitational Waves}

By the mid 1970s scientists were confident that black holes existed (see, for example, 4]), and by the early 1980s we had observed gravitational waves indirectly from the Hulse-Taylor pulsar (see [5]). Humanity has, in a great technological feat, built interferometers capable of 


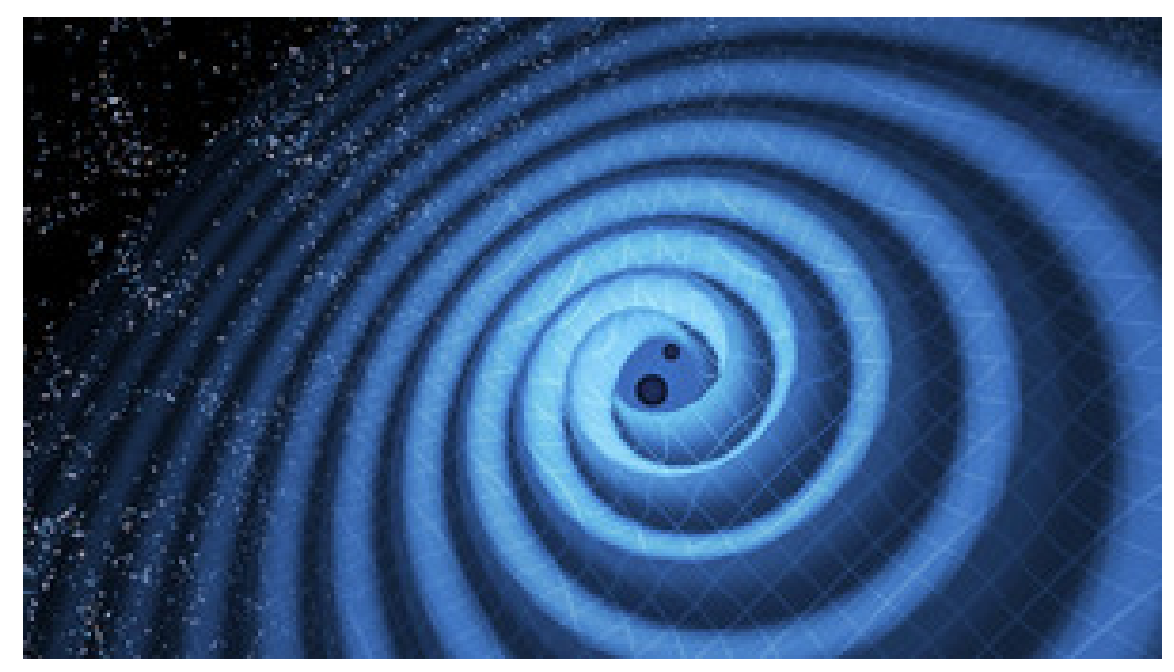

Figure 1.2: An artist's rendition of gravitational waves rippling away from a black hole binary. Image from [3].

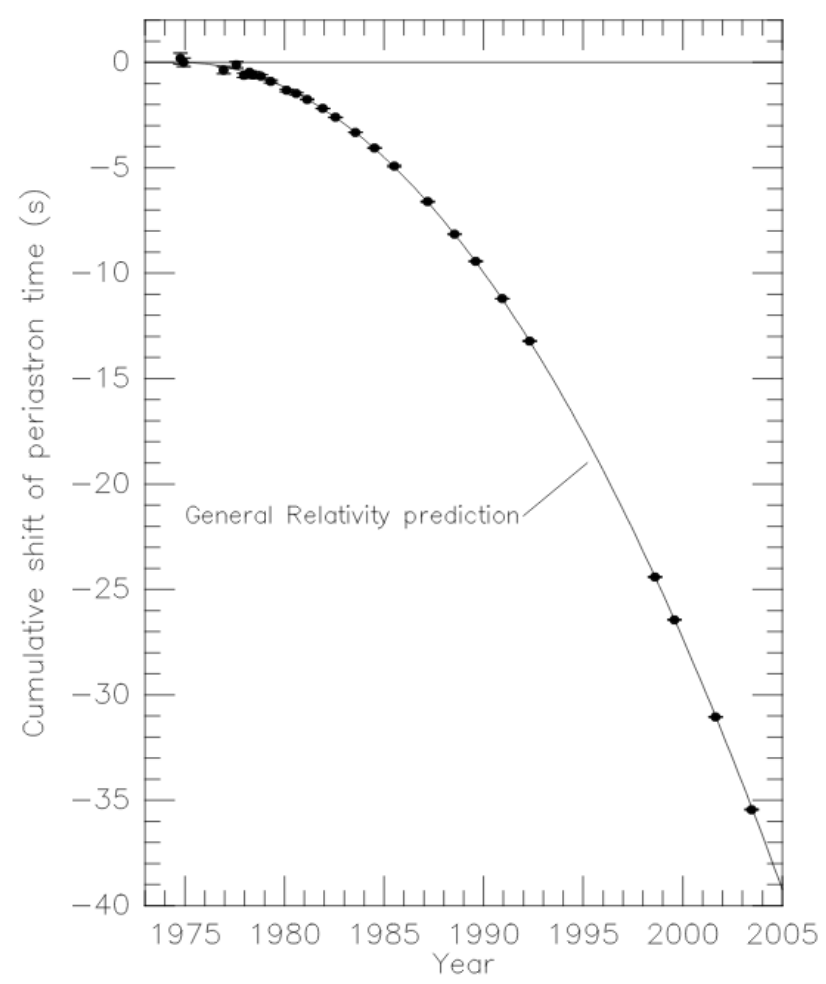

Figure 1.3: Plot of cumulative periastron shift in the Hulse-Taylor pulsar between 1975 and 2005. Note that the line in the plot is the predicted time shift from Einstein's Theory of General Relativity. Image from [2]. 


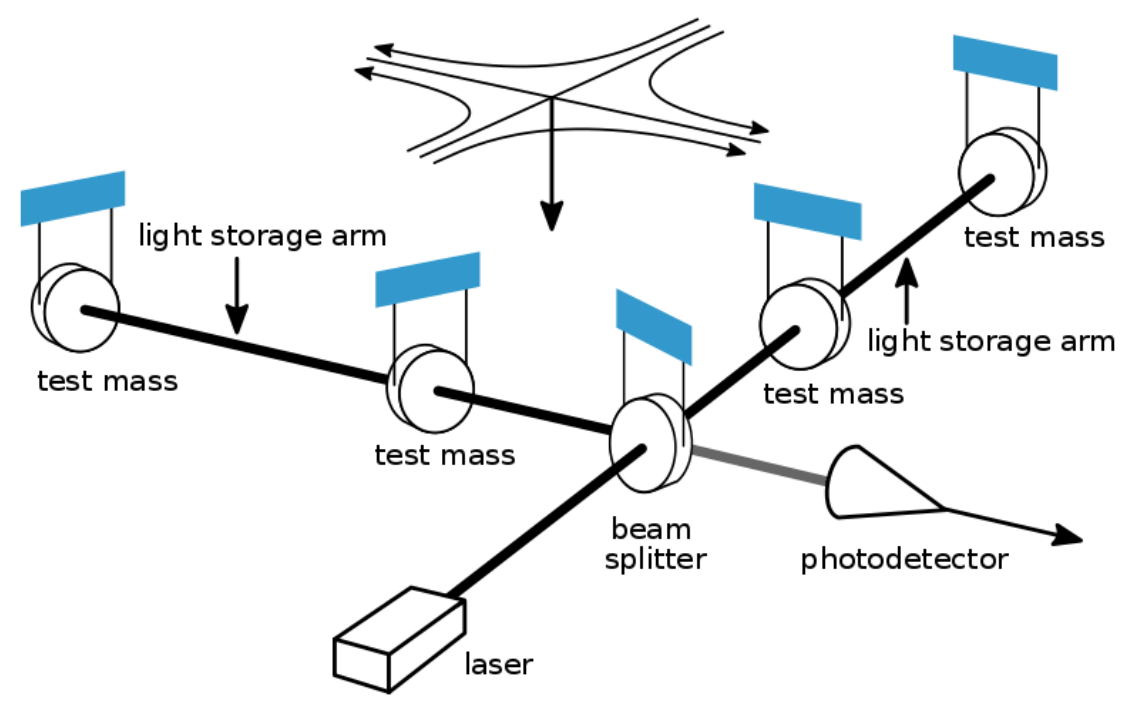

Figure 1.4: Schematic of the LIGO Interferometers. Image from [6]

detecting gravitational waves directly from the late inspiral and mergers of compact celestial objects such as black holes and neutron stars. Two of the most sensitive gravitational wave detectors in the world, the Laser Interferometer Gravitational Wave Observatories ("LIGO"), are located in Livingston, LA and Hanford, WA. A gravitational wave passing through one of these interferometers simultaneously stretches spacetime along one arm and compresses spacetime along the other, causing laser light to constructively and destructively interfere with itself before being recombined and collected by a photodetector (see Figure 1.4 for a schematic representation of the interferometer). To understand the technical achievement required for a successful detection, note that each of LIGO's two, four-kilometer-long arms must be sensitive to stretches or compressions less then one-thousandth the diameter of a proton. The first direct detection of gravitational waves was made by LIGO on 14 September 2015.

The first LIGO detection ushered in a new era of astronomy. Besides "seeing" the Universe in light (along the entire spectrum from microwaves to gamma rays), detecting gravitational waves allows us to "see" another aspect of the Universe: the fabric of spacetime itself. We would like to use this "view" to test other predictions of general relativity and to probe the structure of black holes and neutron stars. Doing so requires that we match detector signals to theoretical waveforms generated on computers. In particular, we seek to 
constrain the eight intrinsic physical parameters of the binary system generating the waves: the mass of each object and the three spin parameters of each object (related to the angular momentum). This process, known as parameter estimation ("PE"), uses a Bayesian framework and is described in Section 2.2. PE can require the sequential generation of up to $10^{8}$ theoretical waveforms to match a single detector signal [7].

Given this description, it is clear that unlocking science from gravitational waves requires efficient algorithms to produce the theoretical waveforms. Unfortunately, in the history of the field of numerical relativity ("NR" — a field dedicated to solving Einstein's equation on computers), only $\sim 10^{3}$ simulations of orbiting black holes have been generated. This is by far too few; in an eight-dimensional parameter space, this provides only $\left(10^{3}\right)^{\frac{1}{8}} \approx 3$ points per dimension. As such, scientists use approximants, built upon these tiny catalogs, to generate theoretical waveforms. Various ways to build approximants are described in Section 2.2, and bringing PE timescales down to the order of months is the motivation for our first paper (which constitutes Chapter 2 of this dissertation). In particular, when initially developed, one of the state-of-the-art gravitational wave approximants (the third version of the spinning effective one body-numerical relativity ("SEOBNRv3") approximant), was about three orders of magnitude too slow for PE. We sped up SEOBNRv3 by two orders of magnitude by applying a number of techniques from numerical analysis, and this approximant has been used to perform $\mathrm{PE}^{1}$.

\subsection{The Way(ve) of the Future}

Much attention has been paid to building approximants using post-Newtonian expansions and fitting parameters to force agreement with NR (see, e.g. [8, 9]). We would like to build, if possible, a physically-motivated approximant (including inspiral, merger, and ringdown) without these fitting parameters. One extremely promising, physically-motivated merger and ringdown model is Dr. Sean McWilliams's "backwards one body" model [10], which

\footnotetext{
${ }^{1}$ Some optimizations to SEOBNRv3 were included early in its development, so that references to SEOBNRv3 in LIGO Scientific Collaboration literature inherently include some of our optimizations to SEOBNRv3. For a list of papers referencing the SEOBNRv3 approximant, see https://tinyurl.com/v3optrefs.
} 
when coupled to the SEOBNRv3 inspiral model has been shown to produce waveforms that agree exceptionally well with NR.

Unfortunately, some core parts of the SEOBNRv3 model are undocumented and poorly understood even by the current maintainers of the code. This clouds the science and hinders future optimizations, which may include rewriting the most algorithmically complex components of the algorithm in more efficient ways. The most time-consuming portion of the SEOBNRv3 algorithm is computing values of the partial derivatives of the Hamiltonian of the binary system. Recall that in Hamiltonian mechanics, the Hamiltonian $\mathcal{H}$ of a physical system is the sum of the kinetic and potential energies in terms of canonical variables $\boldsymbol{p}, \boldsymbol{q}$. The dynamics of the system are given by the solution of an initial value problem, with time evolution equations given by Hamilton's equations of motion:

$$
\begin{aligned}
& \partial_{t} \boldsymbol{p}=-\partial_{\boldsymbol{q}} \mathcal{H} \\
& \partial_{t} \boldsymbol{q}=\partial_{\boldsymbol{p}} \mathcal{H} .
\end{aligned}
$$

This formalism can be extended to relativistic systems, and the SEOBNRv3 approximant solves these equations of motion.

To the end of building a BOB-based approximant, we have fully documented the SEOBNRv3 Hamiltonian. This process is motivated and described in more detail in Chapter 3 .

\subsection{Uncertainty in $G$}

Given the advances in gravitational wave astronomy over the last couple decades, it may seem that scientists understand gravity very well. Strangely, Newton's gravitational constant $G$ remains the one of the most poorly-measured of all physical constants, as it is known to only about 5 significant digits (while, e.g., the mass of an electron is known to about 10 significant digits). Perhaps more surprisingly, some results of various experiments to measure $G$ differ by more than ten standard deviations [11]. Although it is possible that the disparity in these measurements hints at $G$ being non-constant, the general consensus is that the experimental uncertainties of the instruments used to measure $G$ are not well understood. As such, it is crucial that we develop new, innovative experiments for measuring $G$. 


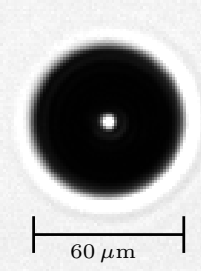

Figure 1.5: Sample data frame for the experiment to measure $G$. The microsphere appears as a dark disk on a light background.

To date, many instruments built to measure $G$ are inspired by the Cavendish torsion pendulum [12]. One new approach to measure $G$ that does not depend on such an instrument (and thereby is independent of its systematic uncertainties) is observing differences in oscillation frequency of a magnetically-levitated microsphere when near and far from symmetric field masses. If such an experiment is to be successful, we must precisely measure the microsphere's position: the more precise the measurement, the better the measurement of $G$. In particular, we measure the microsphere's displacement from some initial position by correlating one frame (a snapshot of the microsphere) with another frame at a later time (a sample frame is shown in Figure 1.5.

Denote by $\mathcal{F}_{0}$ the two-dimensional Fourier transform of an initial frame, and denote by $\mathcal{F}_{i}$ the two-dimensional Fourier transform of an image at time $i$. We compute the cross-power spectrum of these images via

$$
R=\frac{\mathcal{F}_{0} \circ \mathcal{F}_{i}^{*}}{\left|\mathcal{F}_{0} \circ \mathcal{F}_{i}^{*}\right|}
$$

where $\circ$ denotes element-wise multiplication and $*$ denotes complex conjugate. The inverse Fourier transform of $R$ is a surface, the peak location of which indicates the translation between the images. There exist robust phase correlation algorithms allowing for subpixel resolution of image translation, and we were able to combine one such algorithm with a novel method to reduce the effect of background noise in determining the microsphere's position.

Our data consists of a set of equally-spaced-in-time frames made up of two-dimensional translations of the microsphere (which never leaves the field of view). This novel image analysis technique proceeds as follows: we designate the first frame in the series the "zeroth eigenframe". Using the sci-kit image function register_translation(), we can determine 
the position the object in each image relative to its position in the zeroth eigenframe using a standard approach [13]. As described in Section 4.4, this algorithm sometimes yielded jumps in the object's position. We attributed these jumps to (background) noise in the frames, and smoothed the noise in the following way: for each image, use the translation values in the $z$ (horizontal) and $y$ (vertical) directions to overlay the images in the Fourier domain on top of the zeroth eigenframe, compute a global average, and dub this frame the "first eigenframe". We then correlate against this first eigenframe to refine the positions determined by register_translation(). This process can be iterated as many times as we would like. In Chapter 4, we demonstrate that $1.6 \mathrm{~nm}$ precision can be achieved with with new approach. A more detailed overview of the eigenframe approach is given in Appendix B.

At present, the experimental apparatus and systematic error model are not yet in place to measure $G$. We nonetheless discovered that the system proposed to measure $G$ (described in Section 4.3 can, in principle, form a low-mass, room-temperature accelerometer sensitive to low frequencies. The experimental set-up and data analysis that make this possible are detailed in Chapter 4. We include as Chapter 5 a follow-up paper to that in Chapter 4 . which uses the same data analysis technique to show that an optical table can be stabilized with the same mechanism. 


\section{Chapter 2}

\section{Improving Performance of SEOBNRv3 by $\sim 300 x^{1}$}

\subsection{Abstract}

When a gravitational wave is detected by Advanced LIGO/Virgo, sophisticated parameter estimation (PE) pipelines spring into action. These pipelines leverage approximants to generate large numbers of theoretical gravitational waveform predictions to characterize the detected signal. One of the most accurate and physically comprehensive classes of approximants in wide use is the "Spinning Effective One Body-Numerical Relativity" (SEOBNR) family. Waveform generation with these approximants can be computationally expensive, which has limited their usefulness in multiple data analysis contexts. In prior work we improved the performance of the aligned-spin approximant SEOBNR version 2 (v2) by nearly 300x. In this work we focus on optimizing the full eight-dimensional, precessing approximant SEOBNR version 3 (v3). While several v2 optimizations were implemented during its

\footnotetext{
${ }^{1}$ As first author, Tyler D. Knowles drafted this paper and lead the project to optimize SEOBNRv3 with the assistance of coauthors Caleb Devine, David A. Buch, Serdar A. Bilgili, Thomas R. Adams, Zachariah B. Etienne, and Sean T. McWilliams. Note that this version of the paper has been lightly edited and reformatted. Citation: T. D. Knowles, C. Devine, D. A. Buch, S. A. Bilgili, T. R. Adams, Z. B. Etienne, and S. T. McWilliams. Improving performance of SEOBNRv3 by $\sim 300 x$. Classical and Quantum Gravity, 35(15):155003, June 2018 [14].
} 
development, v3 is far too slow for use in state-of-the-art source characterization efforts for long-inspiral detections. Completion of a PE run after such a detection could take centuries to complete using v3. Here we develop and implement a host of optimizations for v3, calling the optimized approximant v3_Opt. Our optimized approximant is about 340x faster than v3, and generates waveforms that are numerically indistinguishable.

\subsection{Introduction}

With its first detections of gravitational waves [15, 16, 17, 18, 19, 20], the Advanced Laser Interferometer Gravitational Wave Observatory (Advanced LIGO) has provided a fundamentally new means of observing the Universe. At the heart of each of these detections was a merger of compact binaries. In such binaries, each compact object possesses four intrinsic parameters: mass, and the three components of the spin vector. Inferring all eight intrinsic parameters $\mathrm{2}^{2}$ from a gravitational wave observation, which analysis is part of the more general parameter estimation (PE), remains a challenging and computationally expensive enterprise.

The LIGO/Virgo Scientific Collaboration (LVC) performs PE in a Bayesian framework, implemented within the LALINFERENCE software package that is part of the larger opensource software framework LALSuite [21]. In such a framework, we sample the posterior distribution by repeatedly calculating the likelihood that a particular waveform matches the data and applying Bayes' theorem. Evaluating the likelihood requires the rapid, sequential generation of as many as $\sim 10^{8}$ theoretical gravitational wave predictions [7]. Generating so many predictions via a full solution of the general relativistic field equations (using the tools of numerical relativity) would be far too computationally expensive. Thus theoretical models adopted for PE generally employ approximate solutions called approximants. State-of-the-art approximants adopt post-Newtonian techniques for evaluating the gravita-

\footnotetext{
${ }^{2}$ Intrinsic parameters are fundamental to the underlying physics of the system. In contrast, extrinsic parameters are related to the observer (e.g. polarization, sky location, and distance) and are not considered in this paper. Some authors refer to seven intrinsic parameters in the full-dimensional space, which include each spin component and the mass ratio of the system. This is because the total mass of the system is simply a scaling factor; we choose to refer to eight parameters since the total mass sets the time and frequency scales and therefore must be considered in PE.
} 
tional waveform throughout most of the inspiral and ringdown, and inject information from numerical relativity calculations for the late inspiral and merger.

One such gravitational wave approximant is the Spinning Effective One Body-Numerical Relativity (SEOBNR) algorithm. This algorithm marries an effective-one body inspiral gravitational waveform approximation - with unknown higher-order terms fit to numerical relativity-generated gravitational wave predictions - to a black hole ringdown model [22]. In particular, SEOBNR starts with the Effective One Body (EOB) approach to non-spinning binary modeling [8] by mapping the dynamics of the two-body system to the dynamics of an effective particle moving in a deformed Schwarzschild metric. This work was then extended to include the effects of spinning, precessing binaries [9]. Implemented numerically, this Spinning EOB procedure adopts a precessing source frame in which precession-induced variations in amplitude and phase are minimized during inspiral, and a source frame aligned with the spin of the final body for matching the inspiral to the merger-ringdown [22].

The other widely adopted approximant within the LVC for PE is the Phenom series of phenomenological waveform models. These waveform models are based on the combination of accurate post-Newtonian inspiral models with late-inspiral and merger phenomenological fits to suites of numerical relativity simulations [23]. More recently, Phenom models have been built to include the effects of precession [24]. In particular, precession effects are included by using post-Newtonian methods to compute precession angles and then "twisting" the underlying non-precessing model [24, 25, 26]. Phenom models are simulated completely in the frequency domain, and therefore simplify some aspects of analysis. The only Phenom model designed to generate gravitational waveform predictions across all eight dimensions of parameter space is PhenomP [24], which was extensively used in the first six detection papers. We remark that Phenom is limited to a relatively small number of numerical relativity simulations against which it has been calibrated, and it is difficult to determine the degree of systematic uncertainty in the model without appealing to another model for comparison.

Evaluating the systematic uncertainties of the Phenom model requires construction of an independent gravitational waveform model with independent systematics, and the SEOBNR family of models is a good candidate for this task. The only SEOBNR model capable of generating theoretical gravitational waveform predictions in all 8 intrinsic dimensions of 
parameter space is the third version of the model, v3; the first and second versions were restricted to aligned-spin cases. In particular, v3 was built to accommodate arbitrary mass ratios, spin magnitudes, and spin orientations and has been calibrated and validated against a variety of numerical relativity simulations [27]. Thus v3 is vital for precessing compact binary merger PE.

Unfortunately, v3 is too currently too slow for PE. A single waveform generation across the LIGO band for, say, a black hole-neutron star system using v3 can take as long as an hour on a modern desktop computer. If LIGO observed a black hole-neutron star system merge, a sequential-gravitational-wave-generation PE would take thousands of years. Attempts to overcome the computational challenge of generating such time-consuming gravitational waveforms include the construction of Reduced Order Model (ROM) approximants. ROMs make use of multidimensional interpolations between sampled points in another underlying approximant. For example, a ROM based on the aligned-spin SEOBNR version 2 (v2) approximant [28, 29] is constructed by first generating an extensive collection of waveform predictions using v2 that adequately samples the 4D parameter space reliably covered by v2. Then to obtain the gravitational waveform at any desired point in parameter space, the ROM simply interpolates within the four dimensions of sampled parameter space. A ROM version of v2 can generate waveforms up to $\sim 3000 \mathrm{x}$ faster than v2 directly [28], which explains in part why ROMs enjoy such widespread use within the LVC for data analysis applications.

While ROMs have been constructed with favorable performance characteristics in alignedspin situations, the cost of generating a ROM grows exponentially with the dimension of the ROM (though see [30] for ideas on combating this using a reduced basis approach). No strategy yet exists that can perform the 8-dimensional (8D) interpolations faster than the 8D approximant; until such a strategy is invented, the most promising way to improve the performance of theoretical waveform generation in the full $8 \mathrm{D}$ parameter space will be to optimize the approximant directly. As a proof-of-principle, we demonstrated that such an approach is capable of improving the performance of the aligned-spin v2 approximant by a typical factor of $\sim 280 \mathrm{x}$ [31]. We call our optimized v2 approximant v2_opt. The precessing (8D) v3 approximant was in development as we independently prepared v2_opt, and thus 


\begin{tabular}{|c|c|c|}
\hline $\begin{array}{c}\text { Base } \\
\text { Approximant }\end{array}$ & $\begin{array}{l}\text { Approx. } \\
\text { Name }\end{array}$ & Description \\
\hline \multirow{2}{*}{$\begin{array}{l}\text { SEOBNRv2 } \\
\text { (spin-aligned) }\end{array}$} & $\mathrm{v} 2$ & Initial SEOBNRv2 implementation? see [32. \\
\hline & v2_opt & \begin{tabular}{l|l} 
Optimized v2 & see 31.
\end{tabular} \\
\hline \multirow{6}{*}{$\begin{array}{l}\text { SEOBNRv3 } \\
\text { (precessing) }\end{array}$} & v3_preopt & Initial SEOBNRv3 implementation, see [22]. \\
\hline & v3 & Partially optimized v3_preopt with bug fixes. \\
\hline & v3_pert & v3 with machine- $\epsilon$ mass perturbation 5 \\
\hline & v3_opt & v3 optimized similarly to v2_opt 5 . \\
\hline & v3_Opt & v3_opt with new optimization strategies 6 . \\
\hline & v3_Opt_rk4 & v3_Opt implementing RK4 rather than RK8 6 \\
\hline
\end{tabular}

Table 2.1: Approximant naming conventions. These conventions apply throughout this paper.

originally contained all the same inefficiencies as v2. This suggests that if the full suite of optimizations we implemented in v2 were incorporated into v3, v3-based PE timescales might drop by two orders of magnitude at least.

This paper documents our incorporation of applicable v2 optimizations into v3, as well as our implementation of innovative new optimization ideas, which together act to speed up v3 by $\sim 340 \mathrm{x}$. Optimization strategies are summarized in Sec. 2.3. Section 2.4 presents code validation tests that demonstrate roundoff-level agreement between v3 and our latest optimized version of v3, designated v3_Opt, along with benchmarks providing an overview of performance gains across parameter space in v3_Opt. For convenience, Table 2.1 defines

\section{all SEOBNR approximants referenced in this paper.}

\footnotetext{
${ }^{3}$ As of publication, the most recent updates to v2/v2_opt are found on commit ID 2cce415 in the LALSuite master branch.

${ }^{4}$ To generate a waveform with v3_preopt, download LALSuite from the archived repository page https: //git.ligo.org/lscsoft/lalsuite-archive/tree/14414694698a2f18c9135445003cade805ad2096 and use approximant tag SEOBNRv3.

${ }^{5}$ As of publication, the most recent updates to v3 and v3_opt are found on commit ID $19 \mathrm{e} 95 \mathrm{~b} 4$ in the LALSuite master branch.

${ }^{6}$ Approximants v3_opt and v3_opt_rk4 were updated to run v3_Opt and v3_Opt_rk4, respectively, on commit ID 1391f77 in the LALSuite master branch.
} 


\section{3 v3_opt: Optimizations migrated from v2_opt}

Optimizations to v3 were performed in two phases. In the first phase, described in Sec. 2.3.1. we migrated to v3 all applicable optimizations developed during the preparation of v2_opt. Sections 2.3 .2 and 2.3 .3 detail the second phase of optimization, outlining new strategies incorporated into v3_Opt.

\subsubsection{Migrated Optimizations}

Here we summarize the optimizations to v2 which were migrated to v3 and thus implemented in v3_opt.

- Switching compilers. Switching from the GNU Compiler Collection (gcc) [33] C compiler to the Intel Compiler Suite (icc) 34] C compiler improves performance by roughly a factor of $2 \mathrm{x}$. It is well-known that the icc compiler often produces more efficient executables than the gcc compiler ${ }^{7}$.

- Minimize transcendental function evaluations. The EOB Hamiltonian equations of motion were hand-optimized by minimizing calls to some expensive transcendental functions such as $\exp (), \log ()$, and pow ().

- Replacing finite difference with exact derivatives. When solving the EOB Hamiltonian equations of motion, v3 computes partial derivatives of the Hamiltonian using finite difference approximations. We replaced these with exact, Mathematica-generated expressions for the derivatives, using Mathematica's code generation facilities - which includes common subexpression elimination (CSE) - to generate the C code [35]. Although this alone acts to significantly speed up v3, in this work we further optimize these Mathematica-generated derivatives.

- Increasing the order of the ODE solver. v3 solves the EOB Hamiltonian equations of motion via a Runge-Kutta fourth order (RK4) ODE solver. After implementing exact

\footnotetext{
${ }^{7}$ We used the following compiler flags when compiling with icc: -fno-strict-aliasing, -xHost, and $-02$.
} 
derivatives, we noticed that the number of RK4 steps needed dropped significantlypresumably due to the effective removal of high numerical noise intrinsic to finitedifference derivatives. We then found that adopting a Runge-Kutta eighth order (RK8) ODE solver resulted in $2 \mathrm{x}$ larger timesteps, so an even larger speed-up was observed.

- Reducing orbital angular velocity calculations. The orbital angular velocity $\omega$ was calculated for each $(\ell, m)$ mode (as defined in 22]) inside the ODE solver. As $\omega$ exhibits no dependence on $\ell$ or $m$, this expensive recalculation was unnecessary and needs only be performed once.

For more details about these optimizations see our v2 optimization paper [31].

\subsubsection{Guided Automatic Differentiation: A more efficient way of generating symbolic derivatives of the Hamiltonian}

After migrating the v2 optimizations described in Sec. 2.3.1 to v3, profiling analyses indicated that approximately $75 \%$ of v3_opt's total runtime was spent computing the v3 Hamiltonian [22] and its partial derivatives with respect to the twelve degrees of freedom (consisting of three spatial degrees $\{x, y, z\}$, three momentum degrees $\left\{p_{x}, p_{y}, p_{z}\right\}$, and three spin degrees for each of the two binary components $\left.i \in\{1,2\}:\left\{s_{i}^{x}, s_{i}^{y}, s_{i}^{z}\right\}\right)$.

In v3, the ODE solver computes these partial derivatives by direct evaluations of the Hamiltonian itself via finite difference techniques [32]. In v3_opt, these numerical derivatives were replaced with Mathematica-generated exact derivatives. Although these exact derivatives unlock significant performance gains, the Mathematica-generated C code was neither particularly human-readable (comprising thousands of lines of code output by Mathematica's CSE routines) nor particularly well-optimized (common patterns were still visible and recomputed in the $\mathrm{C}$ code). Attempts to gain performance through consolidation of all derivatives - as was possible in our optimizations of v2 - proved beyond Mathematica's capabilities when differentiating the v3 Hamiltonian on our high-performance workstations. Therefore, $\mathrm{C}$ codes for all twelve exact derivatives needed to be output separately, resulting in a significant number of unnecessary re-computations. 
We present here our new strategy for computing partial derivatives of the Hamiltonian, called guided automatic differentiation (GAD), which results in a significant reduction in computational cost while ensuring the resulting code is highly human-readable. GAD is based on forward accumulation automatic differentiation, with the advantage of the subexpressions being chosen by hand to minimize the overall number of floating point operations.

The following describes the process of computing a partial derivative of the v3 Hamiltonian $H$ with respect to an arbitrary independent variable $x_{1}$ using GAD. We may write $H$ in the following form, where $I$ is a set of input quantities:

$$
\begin{aligned}
v_{1} & =f_{1}(I) \\
v_{2} & =f_{2}\left(v_{1}, I\right) \\
v_{3} & =f_{3}\left(v_{1}, v_{2}, I\right) \\
& \vdots \\
H & =f_{N}\left(v_{1}, v_{2}, v_{3}, \ldots, I\right) .
\end{aligned}
$$

Here $f_{\ell}$ is the $\ell$ th function of the set of input quantities $I$ and previously computed subexpressions $\left\{v_{0}, v_{1}, \ldots, v_{\ell-1}\right\}$. Although $N \approx 200$ for $\mathrm{v} 3$, for the sake of example we suppose $N=3, I=\left\{x_{1}, x_{2}\right\}$, and

$$
\begin{aligned}
& v_{1}=\sqrt{x_{1}}+a x_{1} \\
& v_{2}=\sqrt{x_{2}}+a x_{2} \\
& v_{3}=\left(v_{1}+v_{2}\right) /\left(v_{1} v_{2}\right) \\
& H=v_{3}^{2} .
\end{aligned}
$$

We demonstrate GAD by taking a partial derivative of $H$ with respect to the independent input variable $x_{1}$. Table 2.2 displays the evolution of this example code under the GAD scheme, which proceeds as follows:

1. We begin with a list of variables and subexpression computations for the Hamiltonian, and translate this $\mathrm{C}$ code into the Mathematica language.

2. We parameterize the terms of each subexpression according to their dependence on $x_{1}$. 


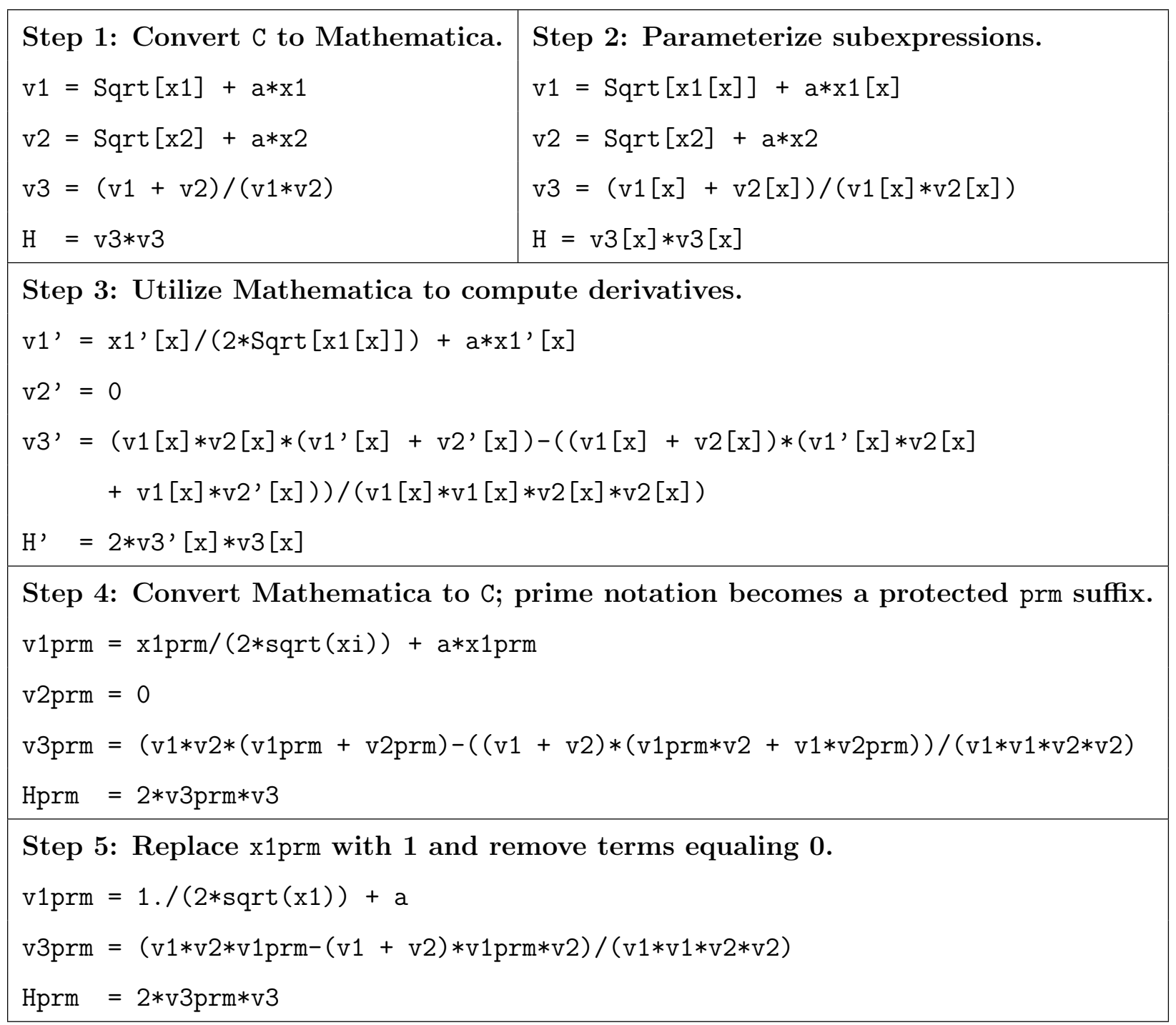

Table 2.2: Step-by-step GAD code evolution.

3. Mathematica computes derivatives of each subexpression.

4. We convert the Mathematica output into $\mathrm{C}$ code.

5. We replace each occurrence of $x_{1}^{\prime}$ with 1 and remove terms equal to 0 .

The resulting $\mathrm{C}$ code is short, optimized, and human readable. Furthermore, any terms that are common to all derivative expressions are computed and saved before computing the partial derivatives, further reducing the computational cost.

Since each $v_{\ell}$ is merely an intermediate of $H$, there is significant freedom in our choice of the set of subexpressions $\mathcal{V} \equiv\left\{v_{1}, v_{2}, \ldots, v_{N-1}\right\}$. Our choices do, however, have a direct effect 


\begin{tabular}{|c|c|c|c|c|c|c|c|}
\hline$a+b$ & $a-b$ & $a * b$ & $a=b$ & $a / b$ & $\operatorname{sqrt}(a)$ & $\log (a)$ & $\operatorname{pow}(a, b)$ \\
\hline 1 & 1 & 1 & 1 & 3 & 3 & 24 & 24 \\
\hline
\end{tabular}

Table 2.3: Relative FLOPs count of the mathematical operations.

on the number of calculations necessary to compute $\partial_{x_{1}} H$, which we measure in floating point operations (FLOP\& $\AA^{8}$ ) Our goal in GAD, therefore, is to choose $\mathcal{V}$ to minimize the number of FLOPs needed to compute $\partial_{x_{1}} H$.

In general, the largest contributor to FLOPs is the product rule. If there are $M$ different subexpressions multiplied together in a given expression, computing the derivative will require $\mathcal{O}\left(M^{2}\right)$ FLOPs. If we therefore choose $\mathcal{V}$ such that each $v_{\ell}$ contains no more than two previous subexpressions multiplied together, we should minimize the overall cost. We expect a significant reduction in FLOPs to correspond to a significant reduction in the time to generate a waveform.

We estimated the number of FLOPs based on benchmarks provided in [36] for CPUs corresponding to the CPU family in our workstations (Intel Core i7-6700) and generated Table 2.3. We emphasize that the values listed in Table 2.3 are truly rough estimates, used only to provide us general direction as we seek an optimal $\mathcal{V}$.

Table 2.4 compares the number of Hamiltonian derivative FLOPs under GAD to the number in the exact derivatives (EDs) generated by Mathematica's CSE code generation algorithm. In principle, the difference in FLOPs between ED and GAD schemes may be used to predict the waveform generation speedup factor. A direct comparison from Table 2.4 indicates a 3.6x reduction in FLOPs when using GAD. For a double neutron star coalescence, Hamiltonian derivative computations constitute about $80 \%$ of waveform generation time. This suggests a speedup factor of 2.3x. Waveform generation times for three scenarios comparing ED and GAD implemented in v3_opt are shown in Table 2.5, and demonstrates a speedup factor of about 1.7x. We emphasize again that counting FLOPs using the relative values of Table 2.3 only provides a rough estimate of the reduction in FLOPs, and the compiler itself rearranges arithmetic expressions to minimize FLOPs as well so the gap

\footnotetext{
${ }^{8}$ Not to be confused with "FLOPs per second" (FLOPS).
} 


\begin{tabular}{|c|ccc|c|}
\hline Derivative scheme & $\begin{array}{c}\text { Space derivative } \\
(\text { FLOPs })\end{array}$ & $\begin{array}{c}\text { Momentum derivative } \\
(\text { FLOPs })\end{array}$ & $\begin{array}{c}\text { Spin derivative } \\
(\text { FLOPs })\end{array}$ & $\begin{array}{c}\text { Total } \\
\text { (FLOPs) }\end{array}$ \\
\hline ED & $3 \times 5073$ & $3 \times 2319$ & $6 \times 4333$ & 48174 \\
GAD & $3 \times 1418$ & $3 \times 527$ & $6 \times 1264$ & 13419 \\
\hline
\end{tabular}

Table 2.4: Number of FLOPs using ED versus GAD methods.

\begin{tabular}{|c|c|c|}
\hline Parameters & v3_opt $(\mathrm{s})$ & $\mathrm{v} 3 \_$opt $(\mathrm{s})$ \\
ED & GAD \\
\hline Neutron Star Binary & 36.75 & 20.49 \\
$1.4 \odot+1.4 \odot, s_{1}^{y}=0.05$ & & $\mathrm{x}(\mathbf{1 . 7 9})$ \\
\hline Black Hole + Neutron Star & 8.07 & 4.69 \\
$10 \odot+1.4 \odot, s_{1}^{y}=0.4$ & & $\mathrm{x}(\mathbf{1 . 7 2})$ \\
\hline Black Hole Binary $(\mathrm{GW} 150914-$ like $)$ & & \\
$36 \odot+29 \odot$ & 0.64 & 0.38 \\
$s_{1}^{y}=0.05, s_{1}^{z}=0.5, s_{2}^{y}=-0.01, s_{2}^{z}=-0.2$ & & $\mathrm{x}(\mathbf{1 . 6 8})$ \\
\hline
\end{tabular}

Table 2.5: Benchmark comparison of ED to GAD strategies. In each scenario, we adopt a $10 \mathrm{~Hz}$ start frequency.

between our estimated and observed speed-ups is not surprising.

\subsubsection{Dense Output: An efficient way of interpolating sparsely- sampled data}

An RK4 ODE solver with adaptive timestep control solves the EOB Hamiltonian equations of motion in v3; thus solutions are unevenly sampled in time. Subsequent analyses require mapping these data into the frequency domain via the fast Fourier transform (FFT), which expects evenly-sampled data. Rather than restricting the integration timestep, v3 uses cubic splines to interpolate the Hamiltonian solutions after RK4 runs to completion. During optimization of v2, the GSL cubic spline interpolation routine was optimized and gave significant performance gains. During optimization of $v 3$, it was discovered that third-order Hermite interpolation made v3_Opt more faithful to v3 (see Section 2.4). Hermite inter- 
polation requires only two function values and the derivatives at those values, which are available at each step of RK8. Thus we may interpolate the sparsely-sampled data to the desired evenly-sampled data "on the fly" during integration. Such an integration routine is called a dense output method [37]. In particular, suppose the RK8 integrator computes the solution $y\left(t_{0}\right)$ and $y\left(t_{1}\right)$ at times $t_{0}$ and $t_{1}$ with timestep $h$ and derivative values $y_{0}^{\prime} y_{1}^{\prime}$. Then for any $0 \leq \theta \leq 1$, we have

$y\left(t_{0}+\theta h\right)=(1-\theta) y\left(t_{0}\right)+\theta y\left(t_{1}\right)+\theta(\theta-1)\left[(1-2 \theta)\left(y\left(t_{1}\right)-y\left(t_{0}\right)+(\theta-1) h y^{\prime}\left(t_{0}\right)+\theta h y^{\prime}\left(t_{1}\right)\right]\right.$.

As this cubic Hermite interpolation routine uses both the solution data and derivative values at each point, it therefore requires only the output of the RK8 integration and no further data storage or function evaluations.

\subsection{Results}

In Sec. 2.4.1 we establish that v3_Opt produces waveforms which agree with v3 at the level of roundoff error. Section 2.4.2 then describes the process of measuring speedup and demonstrates the speedup factor achieved.

\subsubsection{Determining Faithfulness}

Given two waveforms $h_{1}(t)$ and $h_{2}(t)$ (in the time domain), we determine if $h_{1}(t)$ is faithful to $h_{2}(t)$ using the LVC's open-source PyCBC software [38, 39, 40]. This computation depends on the following definitions, which we write in the same form as [41]. The noise-weighted overlap between $h_{1}$ and $h_{2}$ is defined as

$$
\left(h_{1} \mid h_{2}\right) \equiv 4 \operatorname{Re} \int_{f_{l}}^{f_{h}} \frac{\tilde{h}_{1}(f) \tilde{h}_{2}^{*}(f)}{S_{n}(f)} \mathrm{d} f
$$

with $\tilde{h}_{i}(f)$ denoting the Fourier transform of the waveform $h_{i}(t), h_{i}^{*}$ denoting the complex conjugate of $h_{i}, f_{l}$ and $f_{h}$ denoting the endpoints of the range of frequencies of interest, and $S_{n}(f)$ denoting the one-sided power spectral density (PSD) of the LIGO detector noise. We chose $f_{l}=20 \mathrm{~Hz}$ and $S_{n}(f)$ to be Advanced LIGO's design zero-detuned high-power 


\begin{tabular}{|c|c|}
\hline Mass of Object 1 (solar masses) & $m_{1} \in[1,100]$ \\
\hline Mass of Object 2 (solar masses) & $m_{2} \in[1,100]$ \\
\hline Spin magnitude of Object 1 (dimensionless) & $\left|a_{1}\right| \in[0,0.99]$ \\
\hline Spin magnitude of Object 2 (dimensionless) & $\left|a_{2}\right| \in[0,0.99]$ \\
\hline Binary total mass (solar masses) & $m_{\text {total }} \in[4,100]$ \\
\hline Starting orbital frequency $(\mathrm{Hz})$ & $f=19$ \\
\hline
\end{tabular}

Table 2.6: Ranges of values for random input parameters in our faithfulness tests.

noise PSD [42]. For each waveform, $f_{h}$ is the Nyquist critical frequency [37]. We then define the faithfulness between $h_{1}$ and $h_{2}$ to be the overlap between the normalized waveforms maximized over relative time and phase shifts:

$$
\left\langle h_{1} \mid h_{2}\right\rangle \equiv \max _{\phi_{c}, t_{c}} \frac{\left(h_{1}\left(\phi_{c}, t_{c}\right) \mid h_{2}\right)}{\sqrt{\left(h_{1} \mid h_{1}\right)\left(h_{2} \mid h_{2}\right)}} .
$$

Here $t_{c}$ and $\phi_{c}$ denote the coalescence time and phase, respectively. Note that normalization forces $\left\langle h_{1} \mid h_{2}\right\rangle \in[0,1]$, with $\left\langle h_{1} \mid h_{2}\right\rangle=1$ indicating complete overlap (and therefore a perfectly faithful waveform) while $\left\langle h_{1} \mid h_{2}\right\rangle=0$ indicates no overlap (an unfaithful waveform 9 ). For each faithfulness test conducted, we generate a waveform with two different approximants and the same set of input parameters.

We ran 100,000 faithfulness tests for each set of waveform approximants we wished to compare. The input parameters for each test are randomly chosen by PYCBC with bounds as outlined in Table 2.6; these bounds are chosen to capture the relevant parameter space for v3. Note that each of the spin parameters $s_{i}^{x}, s_{i}^{y}, s_{i}^{z}$ are chosen randomly in $(-1,1)$ with the constraint

$$
\sqrt{\left(s_{i}^{x}\right)^{2}+\left(s_{i}^{y}\right)^{2}+\left(s_{i}^{z}\right)^{2}} \leq 0.99, \quad i \in\{1,2\} .
$$

The specific faithfulness runs we conducted were organized as follows. The approximant v3_pert is identical to v3 except $m_{1}$ is replaced with $m_{1}\left(1+10^{-16}\right)$; such a perturbation should result in waveforms that are nearly identical and provides a measure of how sensitive v3 is to roundoff error. Thus faithfulness tests comparing v3 and v3_pert provide a "control" against which we compare the faithfulness of v3_Opt to v3. As another point of comparison,

\footnotetext{
${ }^{9}$ Another common measure in faithfulness tests is mismatch, defined as $1-\left\langle h_{1} \mid h_{2}\right\rangle$.
} 


\begin{tabular}{|c|c|c|c|c|c|c|c|c|}
\hline \multirow[b]{2}{*}{ Comparison } & \multirow[b]{2}{*}{ Compiler } & \multirow{2}{*}{$\begin{array}{c}\text { ODE } \\
\text { tolerance }\end{array}$} & \multicolumn{5}{|c|}{ Number of waveforms with faithfulness } & \multirow{2}{*}{$\begin{array}{c}\text { Counting } \\
\text { Error }\end{array}$} \\
\hline & & & $<0.8$ & $<0.9$ & $<0.95$ & $<0.99$ & $<0.999$ & \\
\hline v3 vs. v3_pert & gcc & $\varepsilon$ & 1 & 5 & 13 & 104 & 399 & \pm 20 \\
\hline (per $10^{5}$ for $10^{6}$ tests) & icc & $\varepsilon$ & 1.0 & 4.2 & 11.5 & 109.0 & 398.2 & \pm 6.3 \\
\hline \multirow[t]{6}{*}{ v3 vs. v3_Opt } & $g c c$ & $\varepsilon$ & 5 & 28 & 136 & 1184 & 5466 & \pm 74 \\
\hline & icc & $\varepsilon$ & 5 & 28 & 135 & 1174 & 5509 & \pm 74 \\
\hline & icc & $\varepsilon \times 10^{-1}$ & 2 & 16 & 44 & 327 & 1510 & \pm 39 \\
\hline & icc & $\varepsilon \times 10^{-2}$ & 0 & 2 & 12 & 143 & 727 & \pm 27 \\
\hline & icc & $\varepsilon \times 10^{-3}$ & 1 & 3 & 8 & 80 & 511 & \pm 23 \\
\hline & icc & $2 \varepsilon \times 10^{-4}$ & 1 & 1 & 2 & 60 & 457 & \pm 21 \\
\hline \multirow[t]{6}{*}{ v3 vs. v3_Opt_rk4 } & $\operatorname{gcc}$ & $\varepsilon$ & 1 & 9 & 35 & 427 & 1529 & \pm 39 \\
\hline & icc & $\varepsilon$ & 0 & 9 & 35 & 420 & 1510 & \pm 39 \\
\hline & icc & $\varepsilon \times 10^{-1}$ & 1 & 7 & 24 & 223 & 926 & \pm 30 \\
\hline & icc & $\varepsilon \times 10^{-2}$ & 0 & 0 & 8 & 114 & 585 & \pm 24 \\
\hline & icc & $\varepsilon \times 10^{-3}$ & 1 & 3 & 8 & 77 & 483 & \pm 22 \\
\hline & icc & $2 \varepsilon \times 10^{-4}$ & 1 & 2 & 3 & 52 & 423 & \pm 21 \\
\hline
\end{tabular}

Table 2.7: Summary of PyCBC faithfulness results. Here $\varepsilon=1 \times 10^{-8}$ and each row reports the results of a run of $\mathbf{1 0 0 , 0 0 0}$ faithfulness tests. icc refers to Intel compiler version 15.5.223, while gcc refers to GNU compiler version 4.9.

we also test v3 (which is RK4-based) against the RK4-based v3_Opt_rk4. For each approximant comparison we compare the effect of increasingly stricter ODE solver tolerance. By default, v3 sets the ODE solver's absolute and relative error tolerances to $\varepsilon \equiv 1 \times 10^{-8}$; we compare faithfulness at tolerances of $\varepsilon, \varepsilon \times 10^{-1}, \varepsilon \times 10^{-2}, \varepsilon \times 10^{-3}$, and $2 \varepsilon \times 10^{-4}$. Finally, we also consider the effect of compiler choice on faithfulness and so conduct faithfulness runs using both gcc and icc. Table 2.7 summarizes the faithfulness tests conducted and their results; the rightmost column displays the counting error $\sqrt{n}$ for the number of waveforms $n$ with $\langle\cdot \mid \cdot\rangle<0.999$.

We comment on the values in Table 2.7. For a couple of parameters for which $\langle\cdot \mid \cdot\rangle<$ 0.8 when comparing v3 to v3_Opt compiled with gcc, one author back-traced a significant difference between v3 and v3_Opt to the ODE stopping condition or the time of maximum amplitude being clearly wrong in v3 but not v3_Opt. In particular, there are some algorithms within v3 that are fundamentally non-robust, and v3_Opt inherits most of these functions. 
The RK8 integration of v3_Opt should be just as accurate as the RK4 integration of v3_Opt_rk4 when the tolerances are equal, but the output from RK8 should be much sparser (by more than a factor of 2) than RK4. Since we observe worse faithfulness with v3_Opt than v3_Opt_rk4, we conclude that most of the truncation error stems from the interpolation of the sparsely-sampled ODE solution to a uniform timestep.

Most importantly, notice that as we make the ODE solver's tolerance $\varepsilon$ stricter (resulting in smaller errors and more finely sampled output data from the ODE solver), the faithfulness between v3 and v3_Opt improves to the level of agreement between v3 and v3_pert. Thus we conclude that v3_opt generates roundoff-level agreement in the limit of $\varepsilon \rightarrow 0$ with errors dominated by interpolation otherwise.

\subsubsection{Performance Benchmarks}

In order to capture the full effect of our optimizations to v3, we compared waveform generation times of v3_Opt with waveform generation times of v3_preopt. In particular, v3_preopt lacks by-hand optimizations of the EOB Hamiltonian implemented in the development of v2_opt; thus unnecessary computations of transcendental functions pow (), $\log (), \operatorname{and} \exp ()$ remain therein. All reported benchmarks were completed on a single core of a modern desktop computer with an Intel Core i7-7700 CPU and 64 GB RAM.

To highlight cases of interest, Table 2.8 summarizes benchmarks of v3_Opt_rk4 and v3_Opt in comparison to v3_preopt for a handful of scenarios of interest to LIGO. The speedup factors are also included, with speedup simply defined to be the ratio of time to generate a waveform with v3_preopt to the time to generate the same waveform with v3_Opt or v3_Opt_rk4.

To demonstrate that the advertised speedup factors of Table 2.8 apply across the parameter space of binaries of interest to the LVC, we completed four benchmark surveys. The first two concern binary black hole systems, one with varying masses and the other with varying spins. The third survey considers mixed binaries (one black hole and one neutron star), and the fourth binary neutron stars. The parameters tested in each run are included in Table 2.9. The results of these surveys are plotted in Figure 2.1 and summarized in Table 2.8 . 


\begin{tabular}{|c|c|c|c|c|}
\hline Physical scenario & $\begin{array}{l}\text { v3_preopt } \\
\text { gcc, (s) }\end{array}$ & $\begin{array}{c}\text { v3_Opt_rk4 } \\
\text { gcc, (s) }\end{array}$ & $\begin{array}{l}\text { v3_Opt } \\
\text { gcc, (s) }\end{array}$ & $\begin{array}{l}\text { v3_Opt } \\
\text { icc, (s) }\end{array}$ \\
\hline \multirow{2}{*}{$\begin{array}{c}\mathrm{DNS}, s_{2}^{y}=0.05 \\
1.3 \odot+1.3 \odot\end{array}$} & \multirow{2}{*}{8618.60} & 98.51 & 42.85 & 21.22 \\
\hline & & $\mathrm{x}(\mathbf{8 7 . 4 9})$ & $\mathrm{x}(\mathbf{2 0 1 . 1})$ & $x(406.2)$ \\
\hline \multirow{2}{*}{$\begin{array}{c}\mathrm{BHNS}, s_{\mathrm{NS}}^{y}=0.05 \\
10 \odot+1.3 \odot\end{array}$} & \multirow{2}{*}{2760.77} & 20.75 & 8.84 & 4.37 \\
\hline & & $x(133.0)$ & $\mathrm{x}(\mathbf{3 1 2})$ & $\mathrm{x}(632)$ \\
\hline \multirow{2}{*}{$\begin{array}{c}\mathrm{BHB}, s_{2}^{y}=0.05 \\
16 \odot+16 \odot\end{array}$} & \multirow{2}{*}{127.71} & 1.70 & 0.90 & 0.46 \\
\hline & & $\mathrm{x}(75.1)$ & $x(140)$ & $\mathrm{x}(\mathbf{2 8 0})$ \\
\hline \multirow{2}{*}{$\begin{array}{c}\mathrm{BHB}, s_{1}^{y}=s_{2}^{y}=0.9 \\
16 \odot+16 \odot\end{array}$} & \multirow{2}{*}{168.13} & 1.75 & 0.91 & 0.46 \\
\hline & & $\mathrm{x}(96.1)$ & $\mathrm{x}(180)$ & $\mathrm{x}(\mathbf{3 7 0})$ \\
\hline \multirow{2}{*}{$\begin{array}{c}\mathrm{BHB}, s_{1}^{y}=s_{2}^{z}=0.9 \\
10 \odot+10 \odot\end{array}$} & \multirow{2}{*}{235.53} & 3.48 & 1.55 & 0.76 \\
\hline & & $\mathrm{x}(\mathbf{6 7 . 7})$ & $\mathrm{x}(\mathbf{1 5 2})$ & $\mathrm{x}(\mathbf{3 1 0})$ \\
\hline \multirow{2}{*}{$\begin{array}{c}\text { BHB, GW150914-like } \\
36 \odot+29 \odot \\
s_{1}^{y}=0.05, s_{1}^{z}=0.5 \\
s_{2}^{y}=-0.01, s_{2}^{z}=-0.2\end{array}$} & \multirow{2}{*}{31.48} & 0.75 & 0.51 & 0.27 \\
\hline & & $\mathrm{x}(42)$ & $\mathrm{x}(\mathbf{6 0})$ & $\mathrm{x}(\mathbf{1 2 0})$ \\
\hline
\end{tabular}

Table 2.8: Benchmarks and speedups of v3_Opt and v3_Opt_rk4 compared to v3.

\begin{tabular}{lcccc}
\hline Ranges & $m_{1}(\odot)$ & $q$ (dimensionless) & $a_{1}$ (dimensionless) & $a_{2}$ (dimensionless) \\
\hline \hline BHB $_{\mathrm{M}}$ & {$[16.7,100.3]$} & {$[1,10]$} & 0.0500001 & 0 \\
$\mathrm{BHB}_{\mathrm{S}}$ & 10 & 1 & {$[-0.95,0.95]$} & {$[-0.95,0.95]$} \\
$\mathrm{BHNS}$ & {$[7,100]$} & $\frac{M}{1.4}$ & {$[-0.95,0.95]$} & 0 \\
$\mathrm{DNS}$ & {$[1.2,2.3]$} & $\frac{M}{m \in[1.2,2.3]}$ & 0.0500001 & 0 \\
\hline
\end{tabular}

Table 2.9: Surveyed parameters: each survey tested 400 parameter combinations, with 20 evenlyspaced values taken in each range indicated. Here $\mathrm{BHB}_{\mathrm{M}}$ indicates the black hole binary mass survey, $\mathrm{BHB}_{\mathrm{S}}$ the black hole binary spin survey, BHNS the black hole neutron star survey, and DNS the double neutron star survey. We define $q \equiv \frac{m_{1}}{m_{2}}$, the ratio of the mass of object 1 to the mass of object 2 . The dimensionless Kerr spins of each object are denoted $a_{1}$ and $a_{2}$, respectively. Each waveform generation started with a frequency of $10 \mathrm{~Hz}$ used a sample rate of 16,384 $\mathrm{Hz}$. 

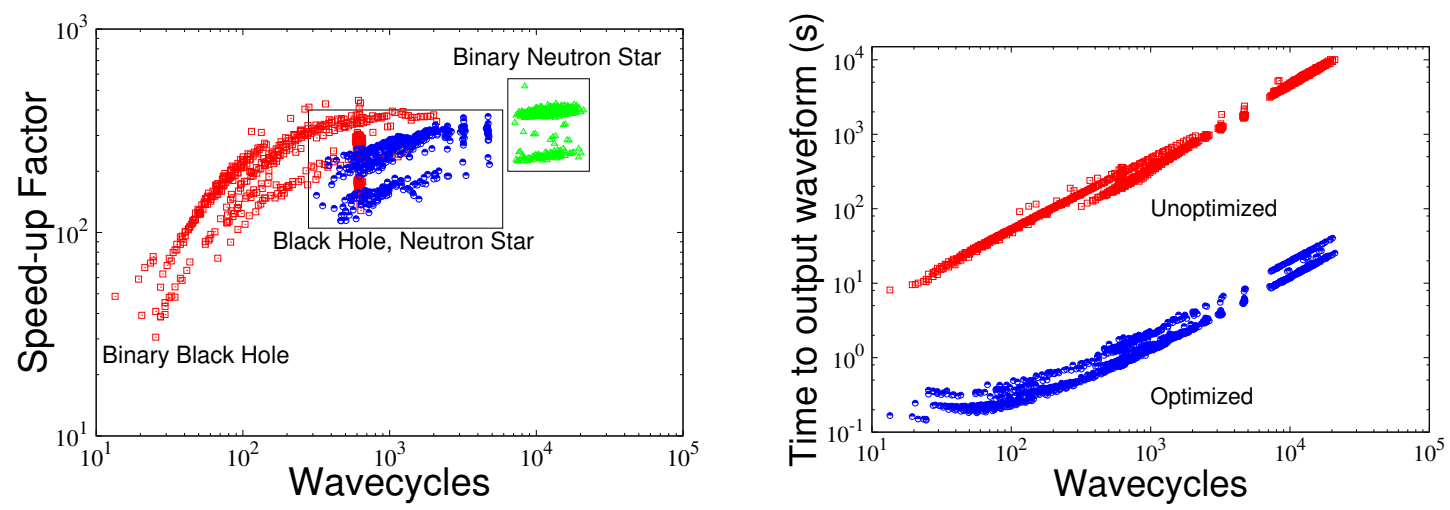

Figure 2.1: Performance benchmarks: Left panel: plots speedup factor versus number of wavecycles in the binary inspiral. Measuring the number of wavecycles allows us to compactly display the results of the benchmark tests without explicit reference to mass or spin. Right panel: plots the number of wavecycles versus the time taken to output the waveform. Note that the speedup factor in the left panel is simply the ratio of the curves in the right panel.

We would like to measure an average speedup based on the four surveys. As in [31], we define an overall speedup factor as a waveform cycle-weighted average

$$
\mathcal{S}=\frac{\sum_{i} \mathcal{S}_{i} N_{i}}{\sum_{i} N_{i}}
$$

where $\mathcal{S}_{i}$ is the speedup factor for generating the $i^{\text {th }}$ waveform and $N_{i}$ is the number of wavecycles in the $i^{\text {th }}$ waveform. We found $\mathcal{S} \sim 340$. This reduces the time necessary for a black hole binary PE run from $~ 100$ years (with v3_preopt) to $\sim 8$ months (with v3_Opt). We expect lower mass PE runs will be possible on similar timescales with additional optimizations.

\subsection{Conclusions and Future Work}

Anticipating the potential detection by Advanced LIGO of significantly precessing compact binaries, we have optimized v3 to make costly precessing-waveform-approximant-based data analysis applications like PE possible in a reasonable amount of time. If an efficient 8D ROM is found, such optimizations will make the construction of this ROM faster. After migrating v2/v4 optimizations to v3, we further optimized partial derivatives of the Hamiltonian using a GAD scheme. This resulted in waveforms that are faithful to v3, as evidenced by faith- 
fulness increasing to 1 as ODE tolerance decreases. We achieved an average overall speedup of $\sim 340 \mathrm{x}$, ranging from $\sim 120 \mathrm{x}$ for GW150914-like black hole binaries to $\sim 630 \mathrm{x}$ for black hole-neutron star binaries. We expect that further optimizations are possible, achieving an additional speedup factor of at least $\sim 3 \mathrm{x}$. Future work will focus on transforming Cartesian coordinates to spherical coordinates to lower sampling rates even more during ODE solving and integration. 


\section{Chapter 3}

\section{Documenting SEOBNRv3 for future approximant optimizations}

Many predictions of General Relativity have been confirmed by experimental evidence. Astronomers and physicists are hopeful that the continued detection of gravitational waves will deepen our understanding of gravity, as well as give insight into the population and structure of black holes and neutron stars. Unlocking knowledge from these detections relies on fast, accurate theoretical gravitational waveforms used to match the detector signals via the process of parameter estimation ("PE").

In particular, we generate these theoretical waveforms by modeling black hole binary systems with parameters $m_{1}, m_{2}$ (masses of black hole one and two, respectively), spin vectors $\boldsymbol{S}_{1}, \boldsymbol{S}_{2}$ (spin vectors of black holes one and two, respectively), and an initial orbital frequency $f$. A schematic of such a system is given in Figure 3.1. The algorithms for simulating these types of systems may also be used to model double neutron star systems and black hole - neutron star systems since the finite-size effects of neutron stars appear at fifth post-Newtonian order (see [43]). To date, research efforts have not focused on modeling effects to that order.

The Laser Interferometer Gravitational Wave Observatory ("LIGO") Scientific Collaboration performs PE in a Bayesian framework, implemented within the LALINFERENCE software package that is part of the larger open-source, data analysis software package LALSuite [21]. Such a framework requires sampling a posterior distribution by repeatedly calculating the 


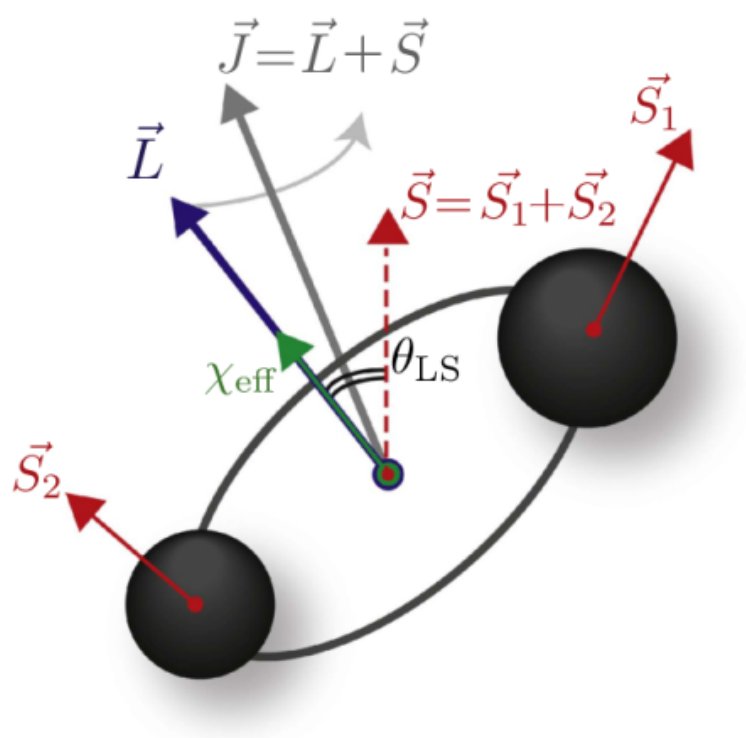

Figure 3.1: Visual of a black hole binary system. Here $\boldsymbol{L}$ is the orbital angular momentum, $\boldsymbol{J}$ is the total angular momentum, and $\chi_{\text {eff }}$ is the projection of the total spin $\boldsymbol{S}$ onto $\boldsymbol{L}$ (usually referred to as the effective spin). When $\boldsymbol{L}$ and $\boldsymbol{J}$ are aligned, we say the binary is "spin-aligned". Otherwise, we say the binary is "precessing". Image from [44].

likelihood that a particular theoretical waveform matches the detector signal and applying Bayes' theorem. Evaluating the likelihood requires the rapid, sequential generation of as many as $\sim 10^{8}$ theoretical waveforms [7].

In approximately the past fifteen years, the field of numerical relativity円("NR") has been able to generate $\sim 10^{3}$ black hole binary calculations. The reason so few calculations have been completed is that these complete numerical solutions to Einstein's equation require significant time to compute on supercomputing clusters. As such, we cannot rely on these calculations for timely PE; instead, we rely on approximate solutions to Einstein's equations called approximants to generate the necessary theoretical waveforms.

LALSuite contains dozens of approximants for PE. One of the fastest families of approximants in LALSuite is called the "Phenom" family of phenomenological approximants. These approximants rely on calibration to numerical relativity ("NR") for merger and ringdown modeling. Understanding the uncertainties associated with such approximants requires

\footnotetext{
${ }^{1}$ Numerical relativity is the field of study that solves Einstein's equation on the computer as an initial value problem.
} 
that we compare the various approximants, including approximants built from physicallymotivated binary models.

Physically-motivated approximants naturally become more complicated and take longer to generate a single theoretical waveform as the models include more relevant physics. One such state-of-the-art family of approximants is the Spinning Effective One Body-Numerical Relativity ("SEOBNR") family of approximants. These algorithms marry an effective-one body inspiral gravitational waveform approximation - with unknown higher-order terms fit to NR-generated gravitational wave predictions - to a black hole ringdown model [22]. In particular, SEOBNR starts with the Effective One Body ("EOB") approach to non-spinning binary modeling [8] by mapping the dynamics of the two-body system to the dynamics of an effective particle moving in a deformed Schwarzschild metric. The first versions of this approximant were "spin-aligned" models, where $\boldsymbol{S}$ in Figure 3.1 is either aligned $(\theta=0)$ or anti-aligned $(\theta=\pi)$. This work was then extended to include the effects of spinning, precessing binaries [9], in which the misalignment of $\boldsymbol{S}_{1}$ and $\boldsymbol{S}_{2}$ causes the orbital plane (and therefore $\boldsymbol{L}$ ) to shift during inspiral. Implemented numerically, this Spinning EOB procedure adopts a precessing source frame in which precession-induced variations in amplitude and phase are minimized during inspiral, and a source frame aligned with the spin of the final body for matching the inspiral to the merger-ringdown [22].

We would like to optimize future versions of the SEOBNR approximants to decrease waveform generation time and improve PE results. There are four basic components to the SEOBNRv3 code: computing initial data, solving the EOB Hamiltonian equations of motion through merger, attaching the ringdown, and generating the theoretical gravitational waveform. Generating the waveform is the core task, but the most time-consuming step is solving the equations of motion which must be done for each waveform generation. This is true both for the spin-aligned approximants (including SEOBNR versions 2 and 4) and the precessing approximants (including SEOBNR version 3, or "SEOBNRv3", described in Chapter 2].

Recall that in Hamiltonian mechanics, the Hamiltonian $\mathcal{H}$ of a physical system is the sum of the kinetic and potential energies in terms of canonical variables $\boldsymbol{p}, \boldsymbol{q}$. The dynamics of the system are given by the solution of an initial value problem, with time evolution equations 
given by Hamilton's equations of motion:

$$
\begin{aligned}
& \partial_{t} \boldsymbol{p}=-\partial_{\boldsymbol{q}} \mathcal{H} \\
& \partial_{t} \boldsymbol{q}=\partial_{\boldsymbol{p}} \mathcal{H} .
\end{aligned}
$$

Computing these equations of motion for the SEOBNRv3 model requires computing the partial derivative of $\mathcal{H}$ with respect to each of the following twelve degrees of freedom: three spatial degrees $\{x, y, z\}$, three momentum degrees $\left\{p_{x}, p_{y}, p_{z}\right\}$, and three spin degrees for each of the two binary components $i \in\{1,2\}:\left\{s_{i}^{x}, s_{i}^{y}, s_{i}^{z}\right\}$. We found that replacing finite difference with exact derivative expressions, along with a host of other optimizations, sped up each of the SEOBNR version 2 and 3 approximants by two orders of magnitude [31, 14]. We expect that similar, and greater, speedups can be achieved for future versions of the SEOBNR approximants.

Unfortunately, some of the SEOBNR code is undocumented; it was developed in an adhoc manner and oftentimes without reference to the relevant theoretical literature. Future optimizations to these approximants, as well as debugging efforts, are delayed by this lack of documentation. For example, consider the file LALSimIMRSpinEOBHamiltonianPrec.c, lines 470 and 473 , in Git commit bba40f21e9 of LALSuite. This file contains the function XLALSimIMRSpinPrecEOBHamiltonian(), which computes the value of the Hamiltonian given a set of spatial coordinates, a value of the momentum vector, and spin vector component values. Though some subterms of the Hamiltonian in this function are prefaced with a comment such as "Eq. 5.70 of BB1" (where BB1 refers to [45]), others are simply prefaced with comments like "Add the additional calibrated term". As calibrated terms are refined or added with each new version of the SEOBNR approximant, such comments are uninformative. To alleviate this issue in the future, we have fully documented the SEOBNRv3 Hamiltonian.

This documentation was completed in a Jupyter notebook [46]. This allows us to both include text (to list paper references, equation numbers, and short explanations) as well as include blocks of Python code that can output the expressions for use in numerical computation. In particular, this SEOBNRv3 Hamiltonian notebook outputs to a text file subterms of the Hamiltonian in SymPy syntax [47]. In the future, we will reduce the number of floating 
point operations required to compute this Hamiltonian by performing common subexpression elimination ("CSE") on these expressions. That is, we will use SymPy to identify common expressions throughout all the subterms and replace them with a variable so that each expression is computed only once. Early testing suggests that SymPy CSE may drop number of floating point operations by as much as 30\% in comparison to the current LALSuite implementation [48].

The general way complicated expressions are written for numerical computations is to start with the most simple, common subterms and build up to the desired expression. Given the complicated nature of the SEOBNRv3 Hamiltonian, the notebook instead begins with the final expression for $\mathcal{H}$ (which we denote by Hreal in the notebook; see Appendix A). From there, we break down $\mathcal{H}$ into subterms (e.g., into terms that include spin-orbit and spinspin coupling), then further break down the subterms of $\mathcal{H}$, etc. until we reach fundamental quantities in terms of mass, position, momentum, and spin. At the end of the notebook, we reverse the order of the subterms for use in numerical computation.

As currently written, the SEOBNRv3 Hamiltonian notebook breaks up the SEOBNRv3 Hamiltonian into about 160 subterms. Each subterm is documented with a link to an arXiv paper and equation number (often referring to [45] and [49]). We began this documentation process by listing the terms in the Hamiltonian as expressed in XLALSimIMRSpinPrecEOBHamiltonian(), and broke down those subterms so that they could be written in about a single line of code that was easily comparable to the equation as written in the relevant literature. This streamlines debugging. We found that one calibration term is not documented (see LALSimIMRSpinEOBHamiltonianPrec.c line 274; this is the calibration coefficient k51, which in the notebook is named $\left.\Delta_{5 \ell}\right)^{2}$

To ensure the accuracy of this SEOBNRv3 notebook, we validated the notebook in the following way: after reversing the terms, we converted the SymPy code to NumPy code (via string manipulations) and input reasonable parameters (masses of 23 and 10 solar masses; spins of $(0.01,0.02,-0.03)$ and $(0.04,-0.05,0.06)$, respectively) and compared the output value to that produced by XLALSimIMRSpinPrecEOBHamiltonian(). Initially finding dis-

\footnotetext{
${ }^{2}$ Even the SEOBNRv3 code maintainers, whom we contacted during the process of documentation, were unable to locate a literature reference for $\Delta_{5 \ell}$.
} 
agreement, we (approximately) bisected by comparing an expression half-way through the notebook to a comparable expression in XLALSimIMRSpinPrecEOBHamiltonian(). If the notebook and LALSuite values agreed, we bisected the remaining code for another comparable term; if not, we compared the specific subterms used to build the disagreeing expression.

After this debugging process was complete, our notebook agreed with XLALSimIMRSpinPrecEOBHamiltonian() to 14 significant digits 3 . We then added a validation cell to the bottom of the notebook that checks both the numerical value of the Hamiltonian when the notebook is run (with the same parameters used for debugging), as well as comparing the SymPy output to a trusted list of subterms. If either the numerical value or the text output differs, the notebook warns the user that the notebook has been edited and will not agree with LALSuite.

The complete SEOBNRv3 Hamiltonian documentation notebook is included in the Appendix A. Future work includes porting the SEOBNRv3 initial condition solver from $\mathrm{C}$ to Python in a Jupyter notebook. This will allow us to generate the SEOBNRv3 inspiral (independent of LALSuite) and merge it with Sean McWilliams's backwards one-body merger and ringdown model [10] for a fast and accurate theoretical waveform model that is physically motivated from start to finish.

\footnotetext{
${ }^{3}$ In double precision arithmetic, which is the standard format for number storage and arithmetic operations on many computers, real numbers are stored using 64 bits. In this base 2 system, the smallest number $\epsilon$ such that $1+\epsilon$ evaluates to 1 is about $1.11 \mathrm{e}-16$. This suggests that the most we can expect any two numerical computations can agree is to 16 significant digits.
} 


\section{Chapter 4}

\section{High Sensitivity accelerometry with a feedback-cooled magnetically}

\section{levitated microsphere ${ }^{1}$}

\subsection{Abstract}

We show that a magnetically levitated microsphere in high vacuum can be used as an accelerometer by comparing its response to that of a commercially available geophone. This system shows great promise for ultrahigh acceleration sensitivities without the need for large masses or cryogenics. With feedback cooling, the transient decay time is reduced and the center-of-mass motion is cooled to $9 \mathrm{~K}$ or less. Remarkably, the levitated particle accelerometer has a sensitivity down to $3.6 \times 10^{-8} \mathrm{~g} / \sqrt{\mathrm{Hz}}$ and gives measurements similar to those of the commercial geophone at frequencies up to $14 \mathrm{~Hz}$ despite a test mass that is four billion

\footnotetext{
${ }^{1}$ As second author, Tyler D. Knowles drafted the Offline Image Analysis section of this paper and oversaw the offline data analysis, including algorithm development. The remainder of this paper was drafted by first author Charles W. Lewandowski, who built the experimental apparatus and oversaw data collection. Two other coauthors, Zachariah B. Etienne and Brian D'Urso, advised Lewandowski and Knowles for the duration of the project and edited the paper. This paper was submitted for publication to Physical Review Applied in March, 2020. Citation: C. W. Lewandowski, T. D. Knowles, Z. B. Etienne, and B. D’Urso. High sensitivity accelerometry with a feedback-cooled magnetically levitated micro-sphere. arXiv preprint:2002.07585, 2020 [50].
} 
times smaller. With no free parameters in the calibration, the responses of the accelerometers match within $3 \%$ at $5 \mathrm{~Hz}$. The system reaches this sensitivity due to a relatively large particle mass of $0.25 \mu \mathrm{g}$, a low center of mass oscillation frequency of $1.75 \mathrm{~Hz}$, and a novel image analysis method that can measure the displacement with an uncertainty of $1.6 \mathrm{~nm}$ in a single image.

\subsection{Introduction}

High sensitivity accelerometry has myriad applications in fundamental and practical fields of physics and engineering. The ability to measure extremely small accelerations and forces has uses in absolute gravimeters [51, 52, 53], inertial navigation [54], tests of quantum gravity [55, 56], gravitational wave detection [20], precision measurements of the Newtonian constant of gravitation [12] and other tests of fundamental physics [57].

Typical accelerometers are based on clamped resonator systems [58, 59, 60]. With cryogenic temperatures, force sensitivities as low as $S_{F}^{1 / 2} \sim 10^{-21} \mathrm{~N} / \sqrt{\mathrm{Hz}}$ are predicted [61]. Using a $\mathrm{Si}_{3} \mathrm{~N}_{4}$ membrane [62], quality factors of $10^{8}$ can be achieved at room temperature with oscillation frequencies of $\sim 150 \mathrm{kHz}$, and thermal noise limited force sensitivities of $S_{F}^{1 / 2} \sim 10^{-17} \mathrm{~N} / \sqrt{\mathrm{Hz}}$ are possible. Mechanical devices have the advantage of typically being extremely compact [63, 64]. Systems with very test large masses, such as LISA Pathfinder, can have acceleration sensitivities of $S_{a}^{1 / 2} \sim 10^{-16} \mathrm{~g} / \sqrt{\mathrm{Hz}}[65]$ where $g$ is standard gravity, $g=9.8 \mathrm{~m} / \mathrm{s}^{2}$. Cold atom interferometry systems have also been proposed for measuring small changes in gravity [66, 67, 68] with acceleration sensitivities as low as $S_{a}^{1 / 2} \sim 10^{-9} \mathrm{~g} / \sqrt{\mathrm{Hz}}[69$, 70].

Levitated systems avoid dissipation associated with the mechanical contact of the resonator with its environment. Force sensitivities of $S_{F}^{1 / 2} \sim 10^{-16} \mathrm{~N} / \sqrt{\mathrm{Hz}}, 10^{-18} \mathrm{~N} / \sqrt{\mathrm{Hz}}$ have been measured with particles in optical traps [71, 172]. Acceleration sensitivities of $S_{a}^{1 / 2} \sim 10^{-10} \mathrm{~g} / \sqrt{\mathrm{Hz}}[73]$ have been reported using a permanent magnet levitated above a superconductor at cryogenic temperatures.

Levitated optomechanical systems in vacuum provide extreme isolation from the environment, making them powerful candidates for high sensitivity accelerometry. The field has 
(a)

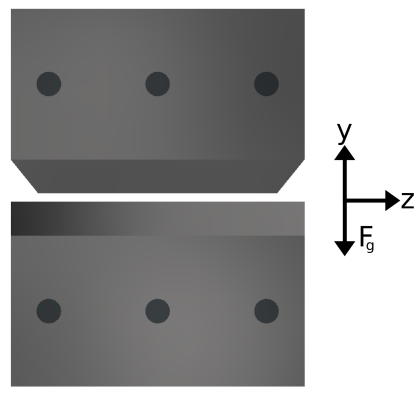

(b)

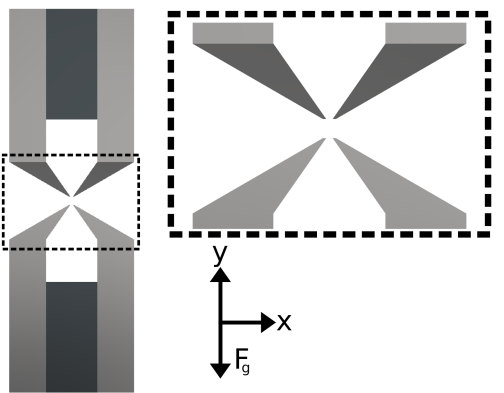

(c)

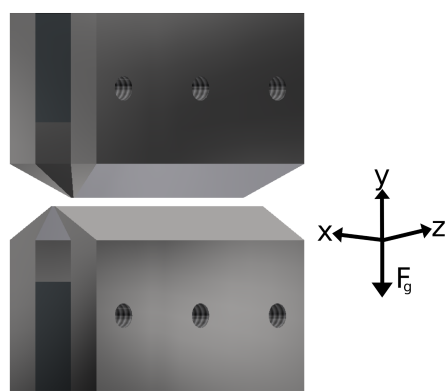

Figure 4.1: (a) The linear magneto-gravitational trap as viewed from the transverse $(x)$ direction. The length of the bottom pole pieces is approximately $26 \mathrm{~mm}$. The top pole pieces are cut shorter to a length of approximately $21 \mathrm{~mm}$. The asymmetry combined with the force of gravity constrains the particle in the axial $(z)$ direction. (b) The view of the trap as viewed from the axial direction showing the quadrupole symmetry in the transverse and vertical (y) directions. (c) A rotated view of the trap showing the quadrupole symmetry and the broken symmetry in the vertical direction. The tapped holes pictured on the pole pieces are used to attach the trap to its mount.

been dominated by optical trapping since its development by Ashkin and Dziedzic [74], in which feedback cooling is typically required for the levitated particle to remain trapped at pressures less than approximately 0.08 Torr [75, 76]. A magnetic trap that does not rely on gravity for confinement has been demonstrated down to a pressure of $\sim 0.1$ Torr [77]. Magneto-gravitational traps have been developed [78, 79] and have exhibited stable trapping to a pressure of $\sim 10^{-10}$ Torr with a feedback cooled center-of-mass motion from room temperature to $140 \mu \mathrm{K}$ [80]. Recent cooling experiments in an optical trap have demonstrated a center-of-mass motion temperature of $50 \mu \mathrm{K}$ for large particles $(\approx 10 \mu \mathrm{m})$ [81. Cooling to the quantum ground state of a sub-micrometer particle has also been shown, reaching a temperature of $12 \mu \mathrm{K}$ from room temperature 82 .

In this paper, we demonstrate levitation of a diamagnetic borosilicate microsphere in a magneto-gravitational trap down to a pressure of $\sim 10^{-7}$ Torr at room temperature. The relatively large mass of the $60 \mu \mathrm{m}$ microsphere and low oscillation frequencies compared to optical trapping systems [83] make this a promising optomechanical system for high sensitivity room temperature accelerometry. The center-of-mass motion is cooled with feedback to damp transients on a reasonable timescale. To check the calibration, accelerations are 
directly applied to the system via a surface transducer.

A critical component of the system is a new offline image analysis technique we have developed to determine the displacement of the trapped particle from photos recording its motion over time. In particular, we mitigate image background noise and avoid issues with fractional pixel translations by constructing a pixel-independent "eigenframe", against which we compute the cross correlation.

\subsection{Experimental Setup}

\subsubsection{Loading and Trapping of Microspheres}

The magneto-gravitational trap, designed with two samarium-cobalt ( $\mathrm{SmCo}$ ) permanent magnets and four iron-cobalt alloy (Hiperco-50A) pole pieces (see Fig. 4.1), creates a threedimensional potential well to stably trap diamagnetic particles. The total potential energy of an object with volume $V$ of diamagnetic material with magnetic susceptibility $\chi$ and mass $m$ in an external magnetic field subject to standard gravity $g$ is

$$
U=-\frac{\chi B^{2} V}{2 \mu_{0}}+m g y,
$$

where $B=|\vec{B}|$ is the magnitude of the magnetic field, $\mu_{0}$ is the vacuum permeability, and $y$ is the vertical displacement of the material [84]. For diamagnetic materials $(\chi<0)$, a stable trap is formed at a magnetic field minimum in the absence of gravity.

The four pole pieces are configured in a quadrupole arrangement surrounding the two permanent magnets. The quadrupole field lies in the transverse-vertical $(x-y)$ plane. Symmetry is broken in the vertical-axial $(y-z)$ plane by cutting the top pole pieces shorter along the axial direction. This asymmetry along with gravity forms the trapping potential in the axial $(z)$ direction [79, 85].

To reduce the effect of thermal noise while maintaining sensitivity to acceleration, larger trapped particles are preferred. A loading method has been developed to allow reliable trapping of large microspheres [80]. In these experiments, we chose borosilicate microspheres (Cospheric BSGMS-2.2 53-63um-10g) with greater than 90\% of particles in the diameter range of $53 \mu \mathrm{m}-63 \mu \mathrm{m}$. Insulating polyimide tape is attached to the tip of an ultrasonic 


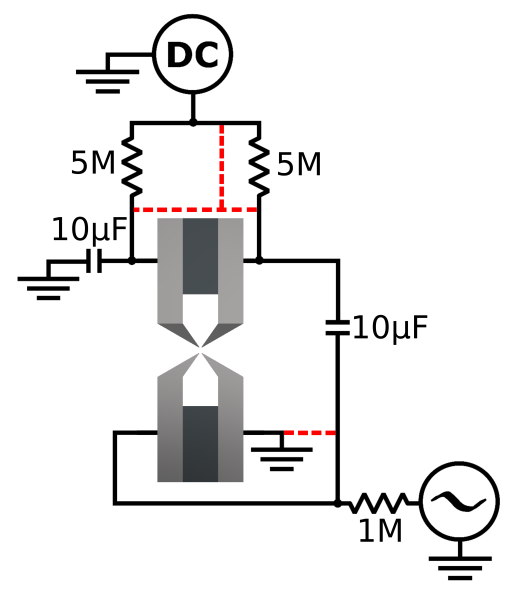

Figure 4.2: A simple schematic of the circuit used to apply the AC voltage for the Paul trap and the DC bias to help counteract gravity. A 50 s filter prevents the addition of high frequency noise. The red dashed lines indicate where jumpers are added to prevent image charge currents from going through high resistance paths when the Paul trap is not in use.

horn [86] to electrostatically hold large microspheres to the tip. The ultrasonic horn shakes the particles off and into the trapping region at atmospheric pressure. An AC voltage is applied to two pole pieces while the other two are kept isolated from the AC voltage to form a linear quadrupole ion (Paul) trap [87, 88] for the particles that have non-zero net charge.

Note that Eq. 4.1 requires a large gradient in $B^{2}$ to balance gravity. The large dimensions of the magneto-gravitational trap form an extremely weak potential. The Paul trap is much stronger, allowing particles to be successfully levitated near the center of the trap. A DC voltage, typically between $20 \mathrm{~V}$ and $40 \mathrm{~V}$, is applied from the top to the bottom pole pieces to help counter gravity and center large particles in the trap.

The DC voltage across the top and bottom pole pieces is supplied from a 1-ppm digital-toanalog converter (DAC, Analog Devices AD5791). The DAC is floated to a voltage between $-300 \mathrm{~V}$ and $0 \mathrm{~V}$ using a modified stacking of Texas Instruments REF5010 high-voltage references [89] in steps of $5 \mathrm{~V}$. The voltage reference circuit can be modified to allow for positive voltages as well. The DAC allows for fine tuning of the voltage, and the resulting potential is estimated to be stable to $<3 \mathrm{ppm}$.

After the particle is loaded into the hybrid Paul-magneto-gravitational trap, the Paul trap is turned off before pumping down the system to high vacuum. The AC voltage is 
slowly decreased while adjusting the DC voltage to keep the particle centered vertically in the magneto-gravitational trap. When the Paul trap is completely off, jumpers, indicated by the dashed lines in Fig. 4.2, are added to eliminate all of the high resistance paths for the movement of image charges.

A mechanical roughing pump along with a turbomolecular pump achieve a pressure of $10^{-7}$ Torr in the vacuum chamber. To eliminate vibrations from these pumps, they are closed off from the chamber and turned off while pumping continues with an ion-sputter pump. A pressure of $\sim 10^{-7}$ Torr was maintained for all measurements reported.

\subsubsection{Table Stabilization}

Changes in the tilt of the optical table cause the equilibrium position of the levitated particle to shift. In the weak direction of the trap, very small changes in tilt can have a significant effect on the equilibrium position. For small tilts, the shift in equilibrium position is described by

$$
\Delta z_{e q} \approx \frac{g}{\omega_{0}^{2}} \Delta \theta
$$

To avoid any large shifts in the equilibrium position, a method has been developed to feedback stabilize the relative tilt of the optical table in real time. The tilt of the table is measured with an ultra-high sensitivity tiltmeter (Jewell Instruments A603-C) and read on a computer. Using two mass flow controllers, air is added or removed from one side of the floating table to keep it level.

Without stabilization, the relative tilt of the table can change by $\pm 150 \mu \mathrm{rad}$ or more. For a levitated particle with an axial oscillation frequency $\omega_{0} /(2 \pi)=2 \mathrm{~Hz}$, this corresponds to a $\pm 9.5 \mu \mathrm{m}$ shift in equilibrium, which is much larger than the typical oscillation amplitudes due to environmental vibrations. With feedback stabilization, this value can be 200 times smaller, resulting in only negligible shifts in equilibrium position.

\subsubsection{Real-Time Image Analysis and Feedback Cooling}

The particle is stroboscopically illuminated using a $660 \mathrm{~nm}$ LED with a repetition rate of $100 \mathrm{~Hz}$ and a pulse duration of $1 \mathrm{~ms}$. As shown in Fig. 4.3, light from the LED is collimated 


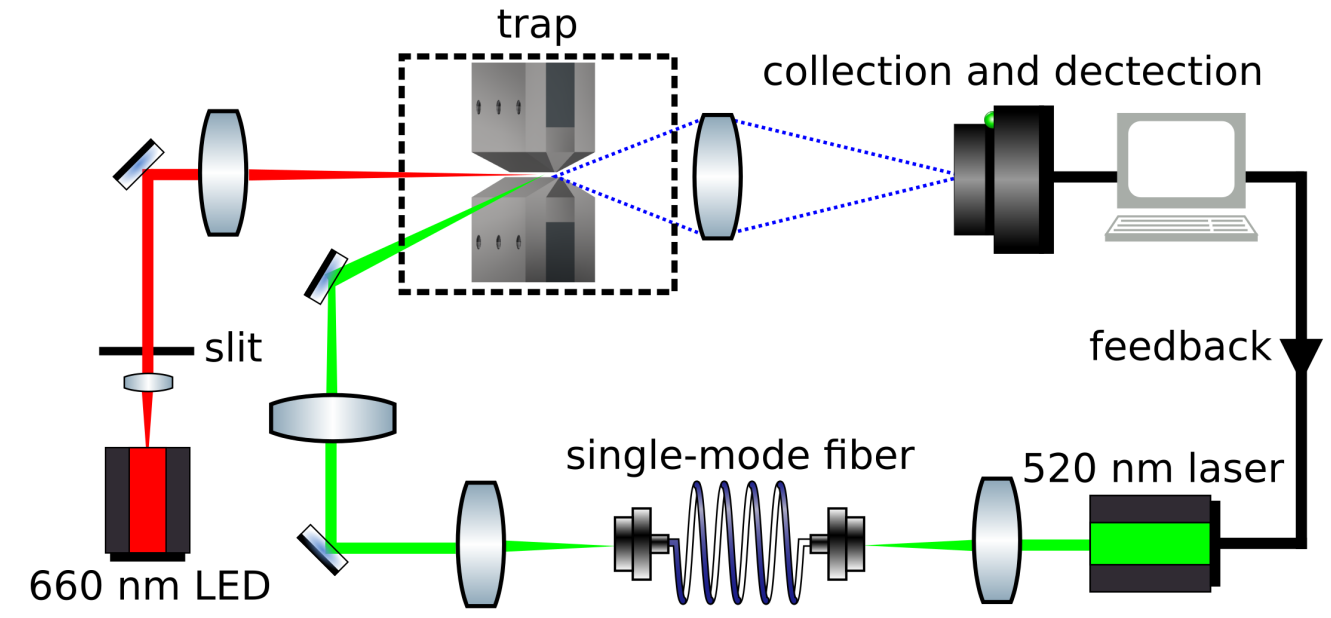

Figure 4.3: Light from a pulsed $660 \mathrm{~nm}$ LED illuminates a slit which is imaged onto the particle, as indicated by the red path. The control laser, indicated by the green path, utilizes a $520 \mathrm{~nm}$ diode laser. Radiation pressure from the laser applies a force that heats or cools the center-of-mass motion of the particle, depending on the phase of the drive relative to the motion of the particle. The scattered green light is blocked by a long-pass filter, while the scattered illumination light is collected and imaged onto a CMOS camera. The images are analyzed in real time to apply the feedback drive to the particle. 
(a)

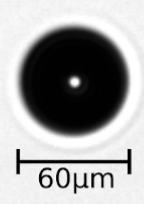

(b)

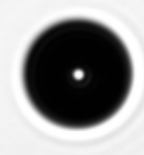

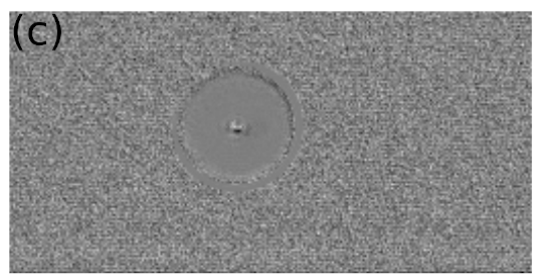

Figure 4.4: ((a) Raw data: dark microsphere on light background with approximate scale. (b) Fifth eigenframe. Note the smoothing of background features in comparison to the raw data. (c) Difference in pixel values between the zeroth eigenframe and the fifth eigenframe. Note that grey pixels denote small differences; dark and light pixels represent the fifth eigenframe having a darker or brighter pixel than the zeroth eigenframe, respectively. Differences in pixel values are scaled by a factor of 9 .

using an aspheric lens and passed through a $100 \mu \mathrm{m}$ slit. The slit is imaged onto the particle and magnified to illuminate the entire region of interest. The particle is imaged onto the CMOS camera with a 0.09 NA telecentric objective (Mitutoyo 375-037-1). All recorded images are 256 by 128 pixels, corresponding to a field of view of $300 \mu \mathrm{m}$ by $150 \mu \mathrm{m}$.

As shown in Fig. 4.4(a), the illuminated microsphere appears as a dark disk in each image (or frame). The microsphere diameter of approximately $60 \mu \mathrm{m}$ corresponds to a diameter of approximately 60 pixels in each frame. The microsphere never leaves the frame in the data we analyze.

The images from the CMOS camera are analyzed in real time to track the motion of the particle. Each image is thresholded to isolate the particle, and the apparent center-of-mass is calculated. The movement from frame to frame is used to calculate the velocity of the particle, which is then passed through a second order infinite impulse response (IIR) peak bandpass filter with a bandwidth of $1.0 \mathrm{~Hz}$ centered at $1.5 \mathrm{~Hz}$ to eliminate high frequency noise.

The measured and filtered velocity of the particle is used to damp and cool the center-of mass motion of the particle via algorithmic feedback [90]. A damping force is applied to the particle using the radiation pressure of the light from a modulated $520 \mathrm{~nm}$ diode laser. Light from this control laser which scatters off the particle is blocked by a long-pass filter before the objective lens used for imaging. 


\subsection{Offline Image Analysis}

If limited to a resolution of one pixel, we could only track the microsphere's position to about $1 \mu \mathrm{m}$. Sophisticated image analysis techniques exist, however, that measure displacement versus some reference frame to a small fraction of a pixel by incorporating all pixel data from each frame. While the image analysis for feedback must be completed in real time, a more accurate but more computationally intensive algorithm can be used for offline analysis of the data. As our first approach, we adopted the cross-correlation function register-translation() available in the scikit-image Python package [13, 91] to determine the displacement of the particle relative to the first recorded frame of data (to which we refer as the "zeroth eigenframe"). While this approach largely seemed to work well, we noticed jump discontinuities in the microsphere displacement versus time as can be seen in Fig. 4.5.(a).

We attributed these discontinuities to noise in the zeroth eigenframe. As this frame was chosen arbitrarily, we anticipated that any other choice of reference frame would result in similar displacement discontinuities. To minimize the effects of this noise we devised a new "eigenframe" approach, which proceeds as follows: we first compute the translation in $z$ and $y$ of each frame against the zeroth eigenframe in the spatial domain using register_translation(). Using these translations, we line up all frames to their inferred displacement with respect to the zeroth eigenframe and construct a globally averaged frame. We refer to the resulting averaged frame as the "first eigenframe". Specifically, the translations and averaging are performed using the two-dimensional discrete Fourier transforms of the images so that the choice of pixel alignment in the spatial domain does not result in loss of information. The averaging smears out the noise present in the zeroth eigenframe and smooths the displacement data (as illustrated in Fig. 4.5(a)). We then refine the translation values by correlating each frame against a translation of the first eigenframe (again in the Fourier domain) to the inferred particle location. The resulting translations may be used to build a second eigenframe in a manner analogous to building the first, and this process can be iterated as many times as we like.

To further refine our position resolution, we modified register_translation() to fit a slice of the correlation surface through the peak in the $z$-direction to a quadratic function 
using SciPy's optimize.curve_fit() function. Locating the peak of this quadratic gives another estimate of the particle translation between each frame and the eigenframe.

To demonstrate that the translation values converge with eigenframe number, denote by $d_{n}\left(t_{i}\right)$ the axial displacement of the microsphere at time $t_{i}$ when correlated against eigenframe $n(n=1,2, \ldots, 5)$. We computed the standard deviation of $d_{n}\left(t_{i}\right)-d_{n-1}\left(t_{i}\right)$ over all $t_{i}$ (see Fig. 4.5(b) and 4.4(c)). Incredibly, the position differences quickly reach a standard deviation of less than $1 \mathrm{~nm}$, thus falling well below the physical resolution limit. After repeating this eigenframe procedure five times, the standard deviation of the change in displacements drops to below $1 \mathrm{pm}$. As this is far below other sources of displacement error in our experiment, the fifth eigenframe is the final one we compute.

\subsection{Acceleration Measurement}

We measure the acceleration sensitivity of the trapped particle by examining the effect of movement of the pneumatically isolated optical table (on which the trap and optics are mounted) on the particle. In the frame of the laboratory, consider the displacement of the particle in the axial direction, $z$, and the displacement of the camera, $z_{0}$. The camera directly measures $z^{\prime}=z-z_{0}$. The equation of motion for the particle in the laboratory frame is then

$$
\ddot{z}+\Gamma \dot{z}+\omega_{0}^{2} z=\Gamma \dot{z}_{0}+\omega_{0}^{2} z_{0}
$$

where $\Gamma$ is the damping rate and $\omega_{0}$ is the resonant angular frequency of the particle.

The displacement of the optical table, for example, from vibrations, can be written as an integral over all frequencies,

$$
z_{0}(t)=\int_{-\infty}^{\infty} d \omega^{\prime} A_{0}\left(\omega^{\prime}\right) \sin \left(\omega^{\prime} t+\phi\right)
$$

where $A_{0}$ is the strength of the drive as a function of frequency.

After substituting Eq. 4.4 into Eq. 4.3, we can take the Fourier transform of Eq. 4.3 , Simplifying the resulting expression, we find that the magnitude of the transfer function is

$$
\left|\frac{Z^{\prime}(\omega)}{A_{0}(\omega)}\right|=\frac{\omega^{2}}{\left(\left(\omega_{0}^{2}-\omega^{2}\right)^{2}+\Gamma^{2} \omega^{2}\right)^{1 / 2}}
$$



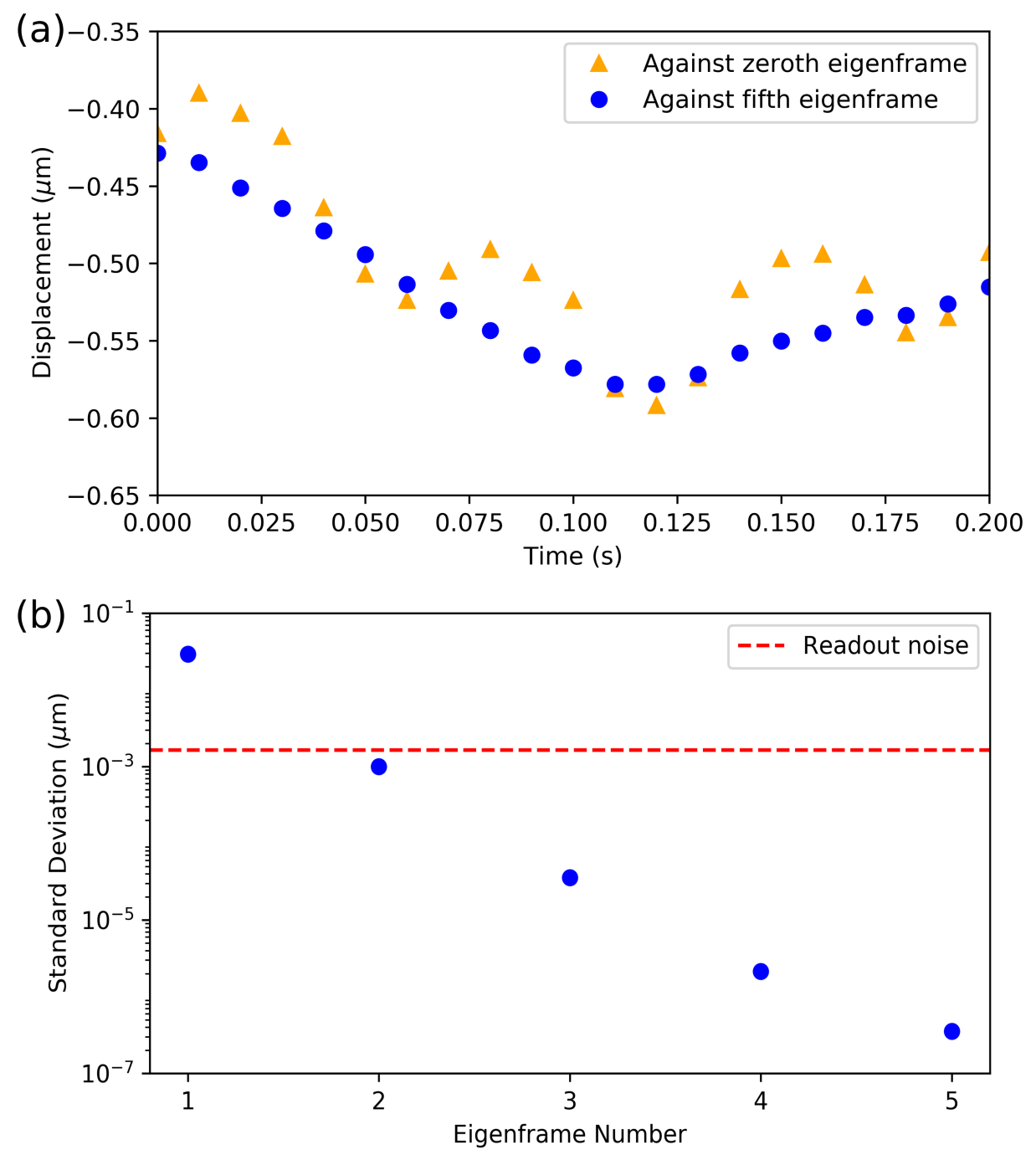

Figure 4.5: (a) Displacement comparison between correlation against the zeroth and fifth eigenframes. Note in particular the discontinuities appearing throughout the zeroth eigenframe displacement time series. (b) Standard deviation of displacement differences between eigenframes $n$ and $n-1$. 


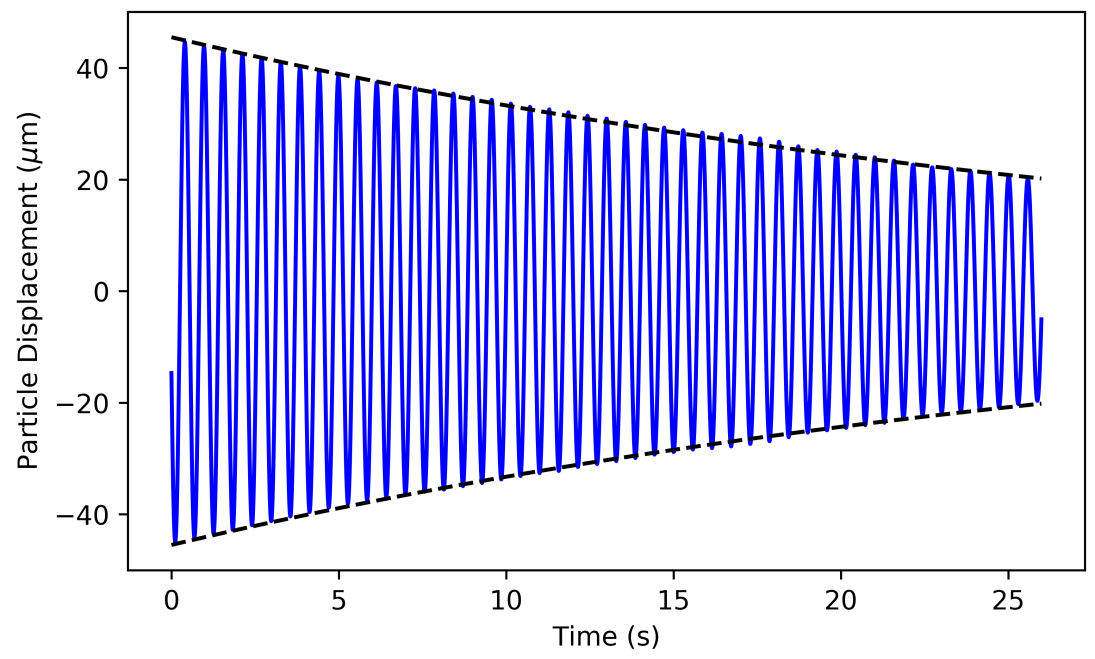

Figure 4.6: Transient motion of the particle after excitation with feedback cooling applied. Analysis of the motion gives an axial $(z)$ oscillation frequency of $\omega_{0} /(2 \pi)=1.75 \mathrm{~Hz}$ and the decay rate is $\Gamma=6.26 \times 10^{-2} \mathrm{~s}^{-1}$ (black dashed lines). The amplitude range plotted and analyzed is chosen so that vibrational noise is negligible.

where $Z^{\prime}(\omega)$ is the Fourier transform of the particle's motion with respect to the camera.

The minimum acceleration that can be detected for an oscillator in thermal equilibrium at temperature $T$ is $[92]$

$$
S_{a}^{1 / 2}=\sqrt{\frac{4 k_{B} \Gamma T}{m}}
$$

where $k_{B}$ is Boltzmann's constant, $m$ is the mass, and $\Gamma$ is the damping rate of the oscillator. Feedback cooling at best keeps $\Gamma T$ constant, damping out potentially long-lived transients without a significant impact on sensitivity 93 .

\subsubsection{Results}

A borosilicate microsphere was levitated with a DC bias across the vertical gap of the magneto-gravitational trap of $-37.2313 \mathrm{~V}$. Throughout the measurements, a vacuum pressure of $10^{-7}$ Torr was maintained and the tilt of the optical table was stabilized to within $\pm 0.75 \mu \mathrm{rad}$. With the measured resonant frequency of the microsphere, Eq. 4.2 gives that the equilibrium position of the particle was stabilized to within $\pm 60 \mathrm{~nm}$.

Before acquiring acceleration data, the system magnification, a critical calibration pa- 

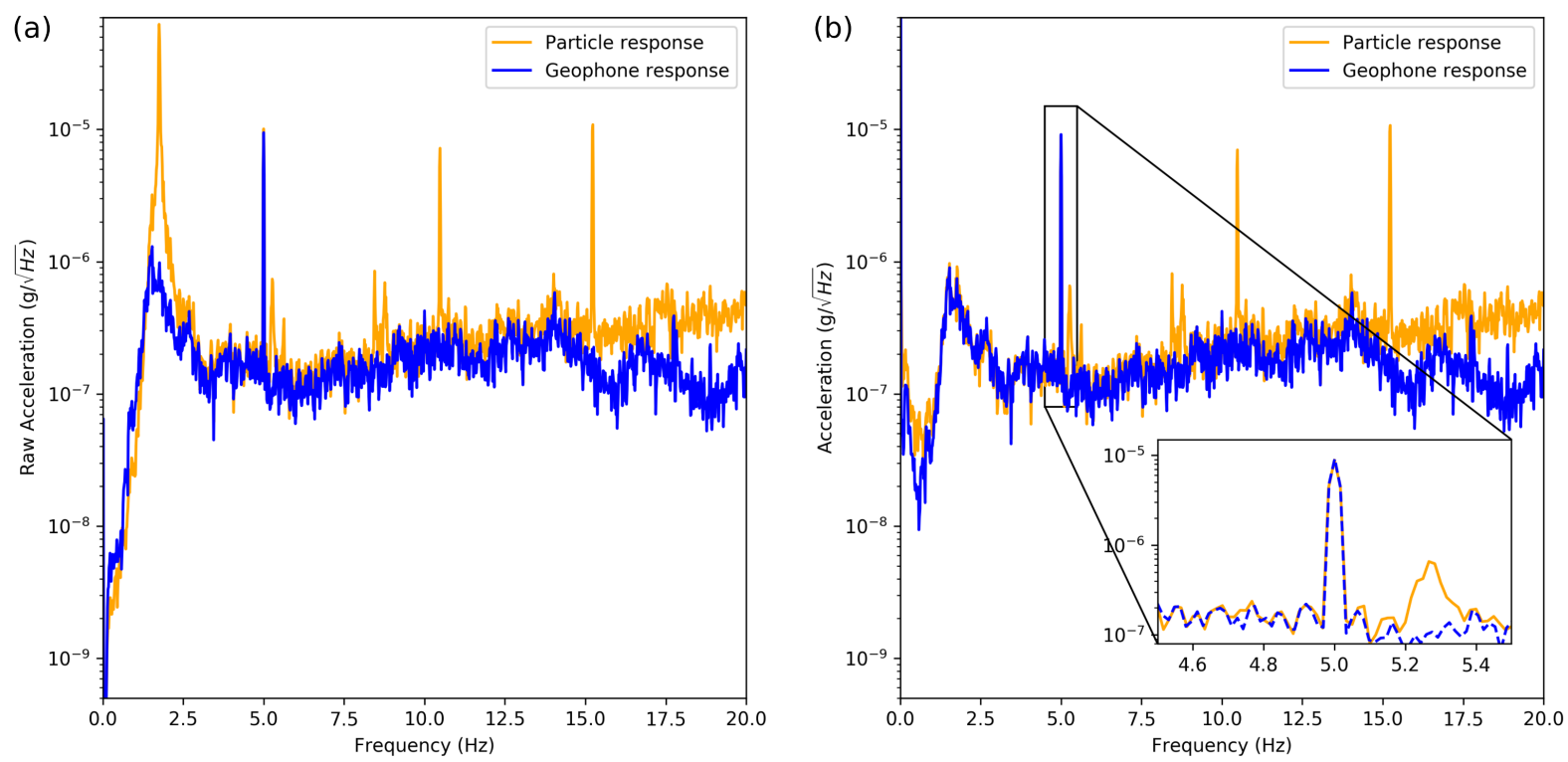

Figure 4.7: (a) The raw data of the particle response in units of acceleration compared to the raw data of the $\mathrm{L}-4 \mathrm{C}$ geophone response in acceleration units with a $5 \mathrm{~Hz}$ drive to verify the calibration. The large peak at $1.75 \mathrm{~Hz}$ is the axial motion and the peaks at $10.5 \mathrm{~Hz}$ and $15.2 \mathrm{~Hz}$ are due to the transverse and vertical motions of the particle, respectively. (b) The particle and geophone responses from (a) divided by their harmonic oscillator responses. The amplitude of the peaks at $5 \mathrm{~Hz}$ differ from each other by less than $3 \%$. The blue line in the inset is dashed so both peaks are visible. 
rameter, is measured. By analyzing the recorded image of a USAF1951 calibration target (Edmund Optics \#58-198) through the system optics, the scaling factor $S_{c}=1.15 \mu \mathrm{m} /$ pixel was determined. For frequency calibration, the digital delay generator used to control all of the timing in the experiment is tied to a rubidium frequency standard (Stanford Research Systems, Inc. FS725).

In order to eliminate any free parameters of the system, the transient response of the microsphere was measured after a small excitation in the axial direction, shown in Fig. 4.6. The resonant frequency of the particle was measured to be $\omega_{0} /(2 \pi)=1.75 \mathrm{~Hz}$. While feedback cooling the center-of-mass motion of the microsphere, the damping rate was measured to be $\Gamma=6.26 \times 10^{-2} \mathrm{~s}^{-1}$.

For comparison, we also place an L-4C geophone (Sercel, Inc. [94]) on the optical table. The sensitivity of this instrument and other critical parameters are given by the manufacturer. We added an additional amplification circuit with a gain of approximately 180 to boost the signal before digitization (modeled after that in [95]).

The response of the particle to movement of the optical table is tested by applying $5 \mathrm{~Hz}$ sinusoidal drive with a surface transducer, oriented to push the table in the axial direction. While applying this external drive, a set of five $60 \mathrm{~s}$ measurements were recorded. Each measurement consists of 6000 images from the CMOS camera, which are analyzed with the algorithm described above. The averaged spectra of the resulting particle acceleration over five data sets is shown in Fig. 4.7(a). For comparison, the measured acceleration of the test mass of the geophone is shown on the same plot. The vibration between $1 \mathrm{~Hz}$ and $2 \mathrm{~Hz}$ is believed to be a resonance of the optical table and overlaps with the resonance of the particle, causing an on-resonance excitation illustrated by the large peak at $1.75 \mathrm{~Hz}$. The transverse and vertical motion of the particle are at $10.2 \mathrm{~Hz}$ and $15.2 \mathrm{~Hz}$, respectively, likely creating peaks at the corresponding frequencies due to misalignments in the system.

To calculate the acceleration of the optical table from the acceleration of the particle and geophone test masses, the harmonic oscillator response of each is divided out of the raw data, resulting in the table acceleration shown in Fig. 4.7(b). The two spectra match over a broad frequency range of approximately $14 \mathrm{~Hz}$. The amplitude of the two peaks at the external drive frequency, $5 \mathrm{~Hz}$, are within $3 \%$ of each other, confirming the calibration between the 


\begin{tabular}{|l|l|l|l|}
\hline Parameter & Description & Geophone Value & Particle Value \\
\hline \hline$T$ & Temperature & $300 \mathrm{~K}$ & $9 \mathrm{~K}$ \\
$\omega_{0} / 2 \pi$ & Resonant frequency of oscillator & $0.97 \mathrm{~Hz}$ & $1.75 \mathrm{~Hz}$ \\
$m$ & Mass of oscillator & $0.957 \mathrm{~kg}$ & $2.5 \times 10^{-10} \mathrm{~kg}$ \\
$Q$ & Quality factor of oscillator & 1.845 & 175 \\
$S_{g}, S_{c}$ & Sensitivity (Note different units) & $281.7 \mathrm{Vs} / \mathrm{m}$ & $1.15 \mu \mathrm{m} /$ pixel \\
$R_{c}$ & Resistance of geophone coil & $5546 \Omega$ & \\
$S_{g}$ & Sensitivity of geophone oscillator & $281.7 \mathrm{Vs} / \mathrm{m}$ & \\
$G_{a}$ & Gain of amplification circuit & 180.2 & \\
$N_{V}$ & Input-referred voltage noise & $8.8 \mathrm{nV} / \sqrt{\mathrm{Hz}}$ & \\
$N_{A}$ & Input-referred current noise & Negligible & \\
$I_{0}$ & Energy density of scattered light & & $2.76 \times 10^{-4} \mathrm{~J} / \mathrm{m}^{2}$ \\
$\Delta z$ & Readout noise & & $160 \mathrm{pm} / \sqrt{\mathrm{Hz}}$ \\
\hline
\end{tabular}

Table 4.1: Critical parameters for the amplified L-4C geophone and levitated particle accelerometers. The geophone values are from the datasheets of the L-4C geophone and OPA188 operational amplifier used in the geophone amplifier.

two systems. Above $14 \mathrm{~Hz}$, the geophone response diverges from the particle response due to increasing noise in the particle acceleration measurement.

\subsubsection{Noise Analysis}

The noise contributions for both the geophone and particle are plotted in Fig. 4.8 along with the (undriven) acceleration of the table as determined by the geophone and levitated particle.

The noise of the L-4C geophone and its accompanying amplification circuit can be broken down into four terms [95]. As displacement equivalent noise sources, they are:

$$
\begin{aligned}
\mathrm{n}_{\text {therm }}(\omega) & =\sqrt{\frac{4 k_{B} T \omega_{0}}{m Q} \frac{1}{\omega^{2}}} \\
\mathrm{n}_{\text {Johnson }}(\omega) & =\frac{\sqrt{4 k_{B} T R(\omega)}}{G(\omega)}
\end{aligned}
$$




$$
\begin{gathered}
\mathrm{n}_{\text {voltage }}(\omega)=\frac{N_{V}(\omega)}{G(\omega)} \\
\mathrm{n}_{\text {current }}(\omega)=\frac{N_{A}(\omega) R(\omega)}{G(\omega)}
\end{gathered}
$$

The thermal noise of the damped harmonic oscillator is given by Eq. 4.7 where $T$ is the temperature of the oscillator, $m$ is the mass, $\omega_{0}$ is the resonant angular frequency, and $Q$ is the quality factor. The thermal fluctuations are approximately $2.4 \times 10^{-11} \mathrm{~g} / \sqrt{\mathrm{Hz}}$ for the geophone with the parameters listed in Table 4.1. The Johnson noise of the geophone coil is given by Eq. 4.8, where $R$ is the real part of the complex impedance of the coil given by

$$
R(\omega)=R_{c}+\frac{i S_{g}^{2} \omega}{\omega_{0}^{2}-\omega^{2}+i \Gamma \omega}
$$

and where $R_{c}$ is the resistance of the coil, $S_{g}$ is the sensitivity of the oscillator, and $\Gamma$ is the damping rate of the oscillator. The harmonic oscillator response $G$ is given by

$$
G(\omega)=\frac{\omega^{3} S_{g}}{\sqrt{\left(\omega_{0}^{2}-\omega^{2}\right)^{2}+\Gamma^{2} \omega^{2}}} .
$$

The input voltage and current noise of the amplification circuit is given by Eq. 4.9 and Eq. 4.10, respectively. $N_{V}$ is the input-referred voltage noise of the OPA188 operational amplifier [96] used in the amplification circuit, assumed to be constant over the frequency range of interest. The current noise of the amplification circuit is negligible compared to all other noise sources for the geophone. The noise sources add in quadrature to give the total noise of the geophone system $\mathrm{n}_{\mathrm{total}, g}$ as

$$
\mathrm{n}_{\text {total }, g}^{2}=\mathrm{n}_{\text {therm }}^{2}+\mathrm{n}_{\text {Johnson }}^{2}+\mathrm{n}_{\text {voltage }}^{2}+\mathrm{n}_{\text {current }}^{2}
$$

The noise of the levitated particle accelerometer has two contributions. First, the thermal noise of the particle is given by Eq. 4.7, where the parameters are now that of the particle (as given in Table 4.1). With feedback cooling applied, we measure the damping rate to determine the $Q$ of 175 , but the effective temperature may be significantly reduced relative to the ambient temperature. Inspection of the minimum signal recorded (around $0.5 \mathrm{~Hz}$ ) puts an upper limit on the noise of $3.6 \times 10^{-8} \mathrm{~g} / \sqrt{\mathrm{Hz}}$ and a limit on the effective temperature associated with the damping of the particle of $9 \mathrm{~K}$. 
The second noise source is readout noise from the camera and image analysis which is expected to be dominated by shot noise of the light and camera noise. To place a lower bound on the readout noise, we consider the precision to which a diffraction limited spot can be determined in the presence of shot noise. This is described by

$$
\left\langle(\Delta z)^{2}\right\rangle=\frac{\sigma_{\mathrm{PSD}}^{2}}{N}
$$

where $\sigma_{\mathrm{PSD}}$ is the standard deviation of the point spread function (PSF) of the imaging optics and $N$ is the number of photons collected, or in our case, blocked, by the particle.

For the lower bound on the noise, the PSF is calculated for a diffraction limited spot. The standard deviation is $\sigma_{\mathrm{PSD}}=0.225 \lambda / \mathrm{NA}$ where $\lambda$ is the wavelength of the scattered light and $\mathrm{NA}$ is the numerical aperture of the collection objective. For our system, $\sigma_{\mathrm{PSD}}=1.65 \mu \mathrm{m}$. The number of photons is estimated from the brightness of the illumination in the CMOS camera and the number of pixels blocked by the $30 \mu \mathrm{m}$ radius particle, resulting in an uncertainty in the location of the point of $\left\langle(\Delta z)^{2}\right\rangle \approx 0.4 \mathrm{~nm}$. Given that the particle is much larger than the diffraction limit, the readout noise is expected to be significantly higher than this.

From $14 \mathrm{~Hz}$ to $50 \mathrm{~Hz}$, the levitated particle acceleration spectrum is not vibration limited. Instead, it follows the expected shape of readout noise, which is a white noise source (in displacement) with the harmonic oscillator response divided out. We fit the spectrum in the frequency range of $35 \mathrm{~Hz}$ to $50 \mathrm{~Hz}$ to find the apparent readout noise of $\Delta z=1.6 \mathrm{~nm}$ per image or $160 \mathrm{pm} / \sqrt{\mathrm{Hz}}$, reasonably above the point source diffraction limit. The two noise sources add in quadrature, so that the total noise of the particle response is

$$
n_{\text {total }, p}^{2}=n_{\text {therm }}^{2}+(\Delta z)^{2} .
$$

\subsection{Discussion}

We have experimentally demonstrated levitation of a $2.5 \times 10^{-9} \mathrm{~kg}$ borosilicate microsphere in high vacuum. This system shows great promise for ultrahigh acceleration sensitivities without the need for large masses or cryogenics. Feedback cooling reduces the transient decay 


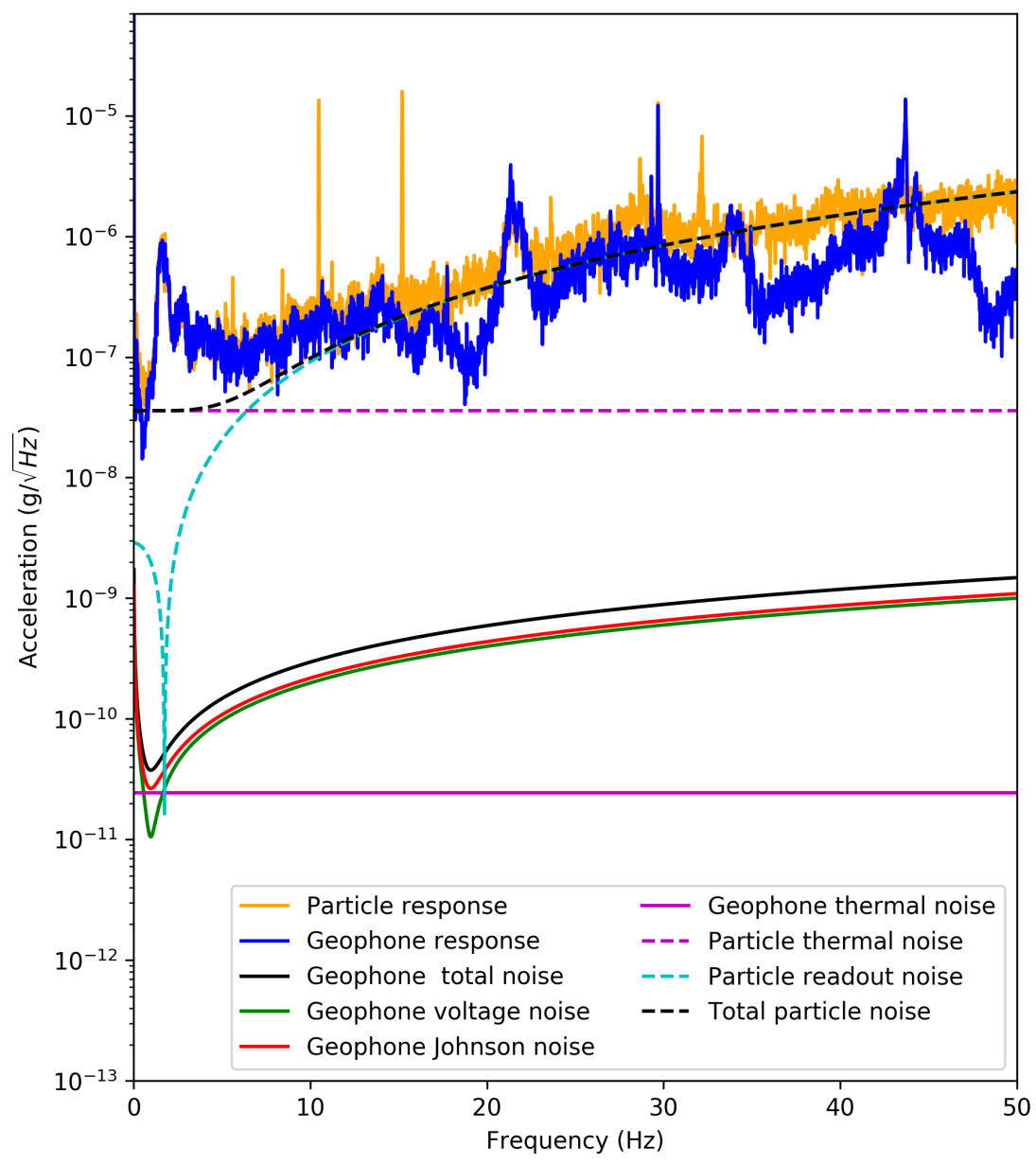

Figure 4.8: The particle and geophone responses divided by the harmonic oscillator responses with no drive. Contributions to the noise for the geophone and particle are also shown. 
time of the system, while also cooling the center-of-mass motion. With no free parameters in the calibration, the acceleration determined from the apparent motion of the particle both follows that of a commercial geophone below $14 \mathrm{~Hz}$ and matches the response to an external drive within $3 \%$ at $5 \mathrm{~Hz}$, despite the particle having a mass that is $4 \times 10^{9}$ times smaller than the test mass in the geophone.

The sensitivity limit in the levitated particle accelerometer is estimated to be below $3.6 \times 10^{-8} \mathrm{~g} / \sqrt{\mathrm{Hz}}$ at low frequencies, limited by either by the vibrations being measured or thermal noise associated with damping at $9 \mathrm{~K}$; a quieter environment would be needed to unambiguously determine the limiting factor and the effective temperature. Much lower center-of-mass temperatures have been reached with trapped particles in other systems, so there is room for significant improvement. For example, feedback cooling to $140 \mu \mathrm{K}$ in a magneto-gravitational trap [80] and $50 \mu \mathrm{K}$ in an optical trap [81] have been demonstrated. Lower center-of-mass temperatures in the current system could result in a sensitivity improvement of at least an order of magnitude, and might be reached by using a more precise real-time image analysis system for feedback cooling. Further improvements are possible using an even lower center-of-mass oscillation frequency or a higher camera frame rate. This high-sensitivity, self-calibrating system with negligible test mass may be particularly valuable for space-based accelerometry at low frequencies.

\subsection{Acknowledgments}

We acknowledge Lin Yi from JPL for discussions on potential mission requirements. This work was supported by the National Science Foundation under awards PHY-1707789, PHY1757005, PHY-1707678, PHY-1806596, and OIA-1458952; the National Aeronautics and Space Administration under awards ISFM-80NSSC18K0538 and TCAN-80NSSC18K1488; and a block gift from the II-VI Foundation. Offline data analysis was completed on the Spruce Knob Super Computing System at West Virginia University (WVU), which is funded in part by the National Science Foundation EPSCoR Research Infrastructure Improvement Cooperative Agreement \#1003907, the state of West Virginia (WVEPSCoR via the Higher Education Policy Commission), and WVU. 


\section{Chapter 5}

\section{Active Optical Table Tilt Stabilization 1$]$}

\subsection{Abstract}

We show that a simple modification to an optical table with pneumatic vibration isolation can be used to actively reduce the long term drift in the tilt of the table by nearly a factor of 1000. Without active stabilization, we measure a root-mean-square (RMS) tilt variation of $270 \mu \mathrm{rad}$ over three days. The active stabilization can be used to limit the tilt to $0.35 \mu \mathrm{rad}$ RMS over the same time period. This technique can be used to minimize drift in tilt-sensitive experiments.

\subsection{Results}

Levitated optomechanics provide a unique system for studying fundamental physics [57] as well as practical applications such as accelerometry [50]. These systems, dominated by optical

\footnotetext{
${ }^{1}$ As second author, Tyler D. Knowles conducted the offline image analysis for this project and edited drafts of this paper. The paper was initially drafted by first author Charles W. Lewandowski, who built the experimental apparatus and oversaw data collection. The coauthors Zachariah B. Etienne and Brian D'Urso advised Lewandowski and Knowles for the duration of the project and edited the paper. This paper was submitted to Review of Scientific Instruments in March, 2020. Citation: C. W. Lewandowski, T. D. Knowles, Z. B. Etienne, and B. D’Urso. Active optical table tilt stabilization. arXiv preprint:2003.03404, 2020. 97.
} 
trapping [74], but also including magnetic traps [85] are typically mounted on optical tables with pneumatic vibration isolation. While the relatively low stiffness of levitated systems makes them highly sensitive force or acceleration sensors [72, 50], it also makes them more sensitive to tilt than clamped resonators (e.g. [98]). In this manuscript, we provide a solution to this issue by actively stabilizing the tilt of the table.

Since trapped particles are typically subject to a harmonic potential, the motion can be described as a simple harmonic oscillator with angular oscillation frequency $\omega=\sqrt{k / m}$, where $k$ is the spring constant of the trap and $m$ is the mass of the trapped particle. For a small tilt of the table $\Delta \theta$, the displacement of the particle $\Delta z$ from the unperturbed equilibrium position is

$$
\Delta z \approx \frac{g}{\omega^{2}} \Delta \theta
$$

where $g$ is the acceleration due to gravity. For low frequency systems, such as those required for precision measurements of the Newtonian constant of gravitation [80], the displacement can be significant enough to disrupt the measurement.

Pneumatic isolators are designed to minimize transmission of vibrations from the floor to the table, but may introduce susceptibility to long term drift in the tilt of the table due to changing environmental conditions. Changes in temperature or atmospheric pressure as well as typical laboratory activities may lead to unacceptable changes in the tilt of the table. In this study, the table used is 8 feet by 4 feet in the $x$ and $z$ directions, respectively, supported by four TMC Gimbal Piston Isolators.

The original table support system consists of a regulator-valve system on each of the three master legs, labeled by $\mathrm{R}$ and $\mathrm{M}$ in Fig. 5.1, respectively. The slave leg, labeled S, is tied to one master leg. In our case, the master-slave pair is parallel to the $x$ direction. The $z$ direction, which we stabilize, is parallel to the short side of the table. Under normal operation, the regulators add or remove compressed air from the isolators to keep the table approximately level.

To stabilize the tilt, a simple modification was made to the regulation of the isolators (see Fig. 5.1). An air line inserted in the master-slave leg pair is immediately split into two lines and connected to two MKS mass flow controllers (MFCs) (MKS 1179C01312CR1BV). One MFC is oriented to allow gas flow towards the legs and the other is oriented to allow gas 


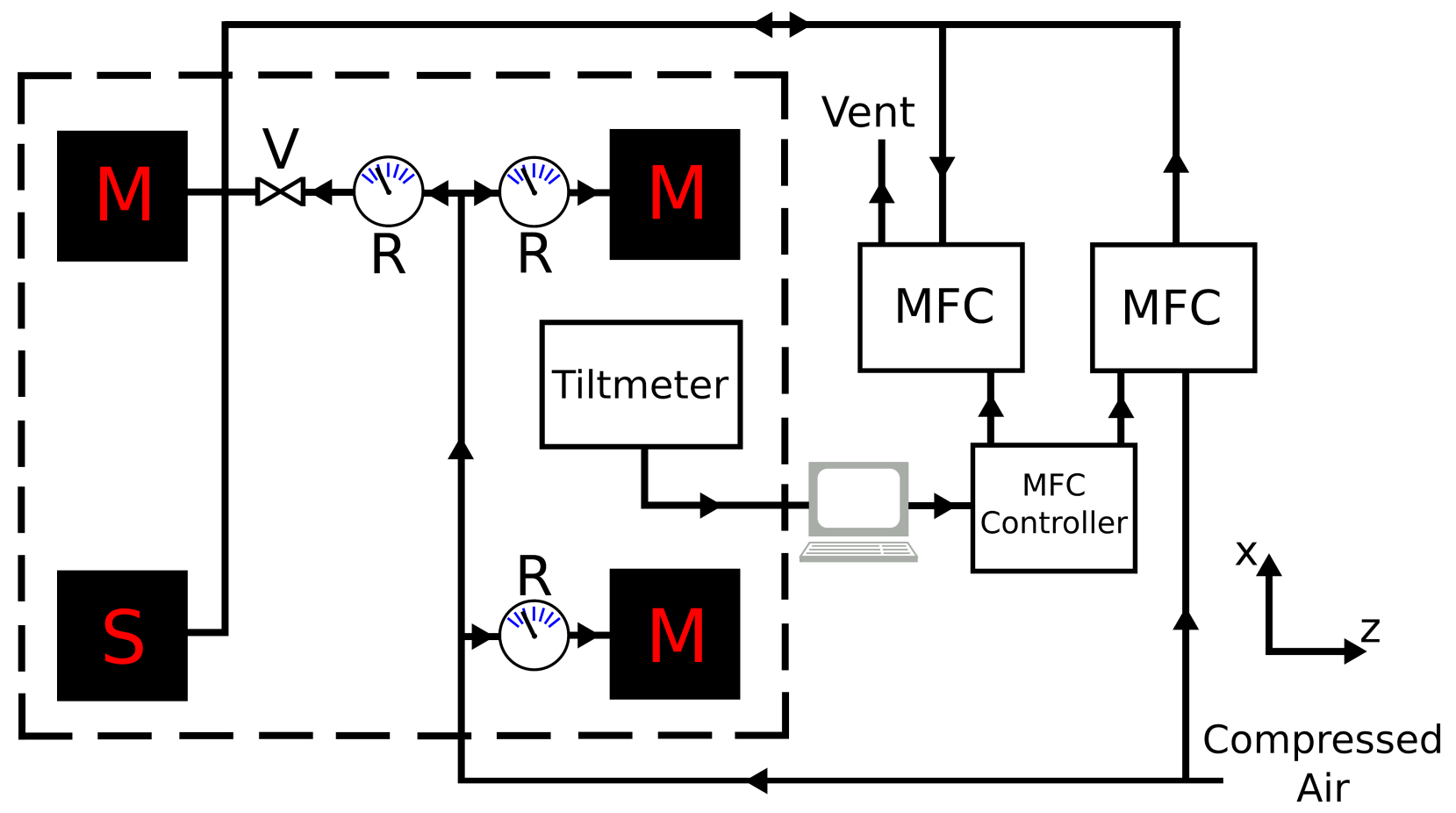

Figure 5.1: System diagram for actively stabilizing the tilt of an optical table. Two mass flow controllers are regulated to add or remove compressed air from the master-slave leg pair, based on real-time readings from the tiltmeter. When stabilized, valve $\mathrm{V}$ is closed to the table regulator. The arrows indicate the direction of flow. 


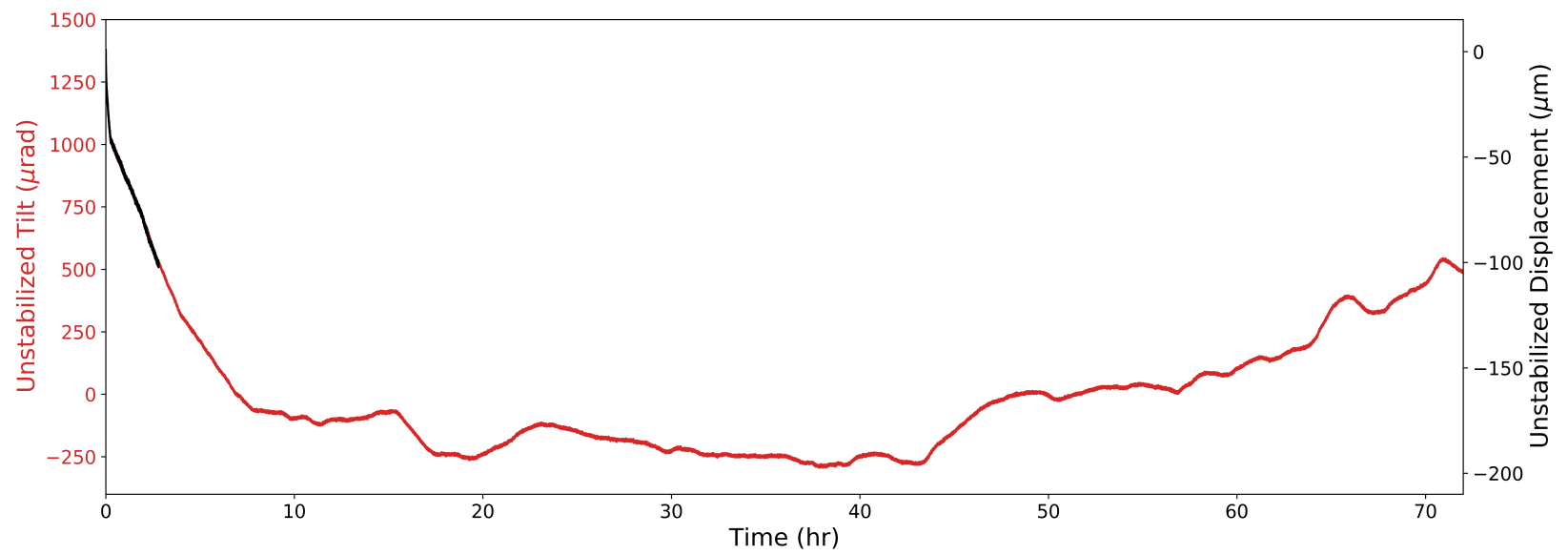

Figure 5.2: The relative tilt of the optical table (red) and displacement of a trapped particle (black) without active stabilization over three days. The particle displacement follows the shape of the relative tilt before the particle leaves the frame of the camera.

to flow out of the legs. The two MFCs are controlled by an MKS type 247 4-channel flow controller power supply and readout (MFC controller). An inline valve is inserted between the master-slave leg pair and its original regulator. The valve isolates the master-slave leg pair from the original regulator to prevent the regulator from competing with the flow from the MFCs when active stabilization is used.

A Jewell Instruments A603-C two-axis tiltmeter is placed on the optical table, centered in the $z$ direction, to monitor the tilt with a resolution of less than 2.5 nrad along two perpendicular axes. The high gain setting with angular range of $\pm 40 \mu \mathrm{rad}$ and an optional $7 \mathrm{~s}$ output filter were used. In the initial setup of the meter, the two axes are oriented along the $x$ and $z$ directions of the table. The outputs are monitored on Keithley 2000 digital multimeters (DMM). The tiltmeter is adjusted by the worm-gear feet until the voltage output is near zero, corresponding to zero absolute tilt. Since we stabilize the relative tilt, it is not crucial to completely zero the tiltmeter.

A second tiltmeter is placed on the optical table, also centered in and aligned with the $z$ direction for coarse tilt detection when the A603-C does not provide enough angular range. The second is a Jewell Instruments LSOC-1Z fluid damped single-axis inclinometer with a $\pm 0.017 \mathrm{rad}$ range and $1 \mu \mathrm{rad}$ resolution. The single $\pm 5 \mathrm{~V}$ output is monitored on a Keithley 2000 DMM. 


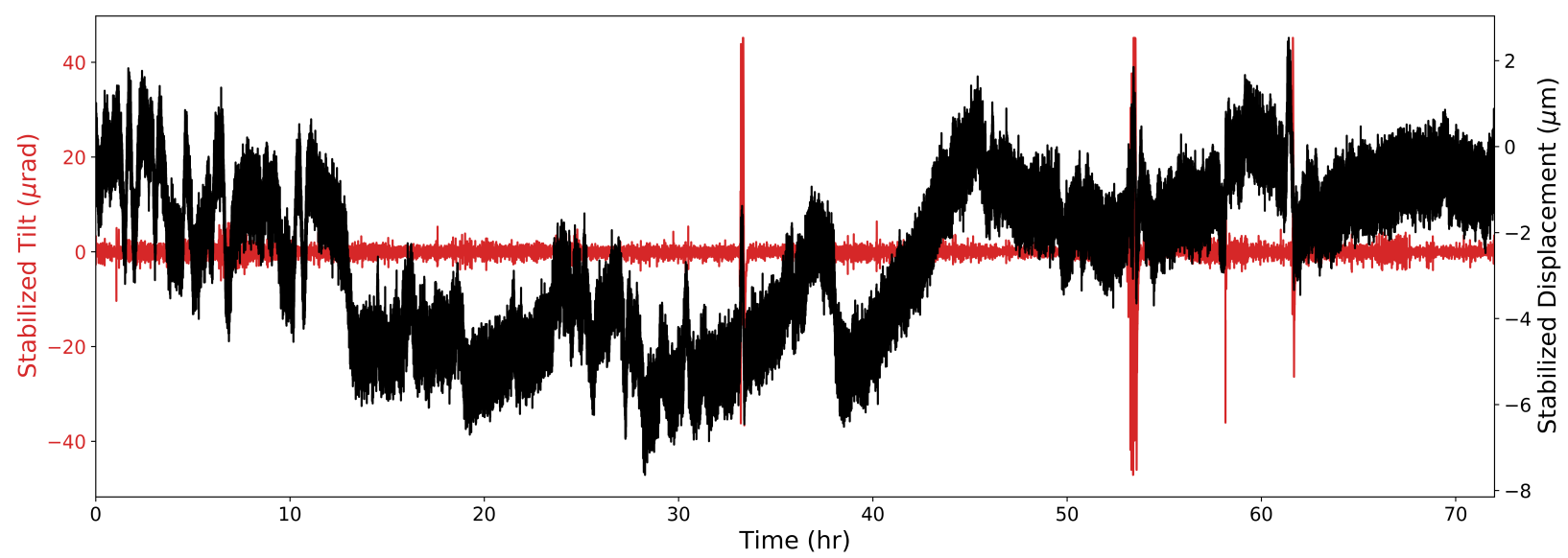

Figure 5.3: The relative tilt of the optical table (red) and displacement of a trapped particle (black) with active stabilization over three days.

The in-flow and out-flow rates are governed by a digital proportional-integral (PI) controller. For every new measurement, the difference between the measured tilt and target tilt, $\Delta_{i}$, is calculated. This error is multiplied by the proportional and integral constants, $P$ and $I$, respectively. The algorithm is described by

$$
F_{i}=P \Delta_{i}+I \sum_{n=0}^{i} \Delta_{n}
$$

where $F_{i}$ is the feedback flow corresponding to the $i^{\text {th }}$ tilt measurement. To prevent excessive integral windup of the algorithm, the integral term is limited to $\pm 216 \mu \mathrm{rad}$. To avoid an abrupt crossover from one mass flow controller to the other (when the sign of $F_{i}$ changes), the outputs were biased such that an equal (but nonzero) flow of gas passed through both MFCs when $F_{i}=0$.

The tilt of the table was monitored for $72 \mathrm{hrs}$ with one tilt reading taken every second, without and with active stabilization. Data without active stabilization was taken after the table was settled under active stabilization. The feedback MFC controller was then turned off and the valve to the regulator on the master-slave leg pair was opened so the table was fully controlled by the table regulators. As shown in Fig. 5.2 , the tilt immediately began drifting and settled over many hours, eventually drifting back in the opposite direction. The root-mean-square (RMS) tilt variation was $270 \mu \mathrm{rad}$, requiring the use of the coarse (LSOC1Z) inclinometer. This not only demonstrates the drift of the tilt over time, but also how 


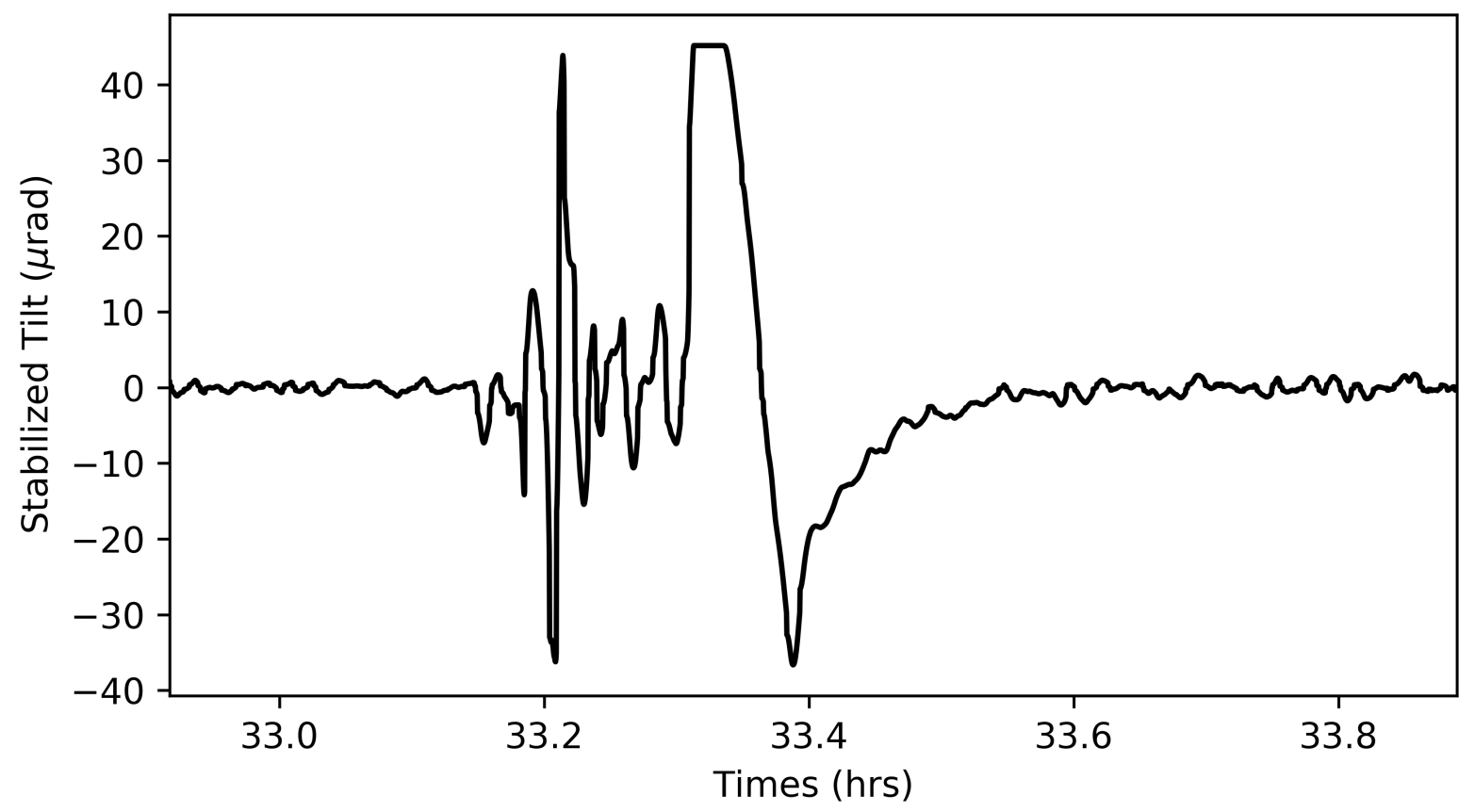

Figure 5.4: The stabilized data of an unknown event occurring at approximately $33.3 \mathrm{hrs.} \mathrm{The}$ relative tilt of the optical table exceeds the range of the A603-C tiltmeter. The entire event lasts $\sim 1000$ s before the stabilization corrects it.

it can take the isolators to initially settle.

To measure the tilt variation with active stabilization, the valve between the original regulator and master-slave leg pair was closed and the table was allowed to stabilize for several minutes before recording the data shown in Fig. 5.3. There are several notable features in the stabilized data occurring at e.g. $33.3 \mathrm{hrs}$. A detailed plot of this event is shown in Fig. 5.4. The cause of the disturbance is unknown but illustrates the performance of the stabilization. Before $33.1 \mathrm{hrs}$, the optical table is well stabilized to within $\pm 1.2 \mu \mathrm{rad}$. After some overshoot, the disturbance is corrected within $1000 \mathrm{~s}$. Over the three day period, the RMS tilt variation was $2.8 \mu \mathrm{rad}$, including the large disturbances.

The effect of uncontrolled tilt on levitated optomechanics experiments can be dramatic. Particles in magneto-gravitational traps can have particularly low frequencies (we have demonstrated down to $\approx 0.1 \mathrm{~Hz}$ ), making them particularly susceptible to tilt (see Eq. 5.1). In this experiment, we trap $60 \mu \mathrm{m}$ diameter borosilicate microspheres in a trap that is $20 \mathrm{~mm}$ long in the $z$ direction. Air at atmospheric pressure is left in the trapping chamber to provide 
damping.

To measure the effects of tilt, images of a microsphere in a trap on the optical table were recorded with a CMOS camera while taking tilt data. The images were analyzed using the eigenframe method to obtain sub-pixel [50] displacement of the particle. The analysis was performed offline after all frames were recorded. The recorded image is approximately $250 \mu \mathrm{m}$ horizontally and $125 \mu \mathrm{m}$ vertically, typical for our experiments.

The displacement of the particle during the tilt measurement without active stabilization is shown by the black curve in Fig. 5.2. The oscillation frequency of the particle is inferred by adjusting the scale appropriately for the particle displacement to match the relative tilt, according to Eq. 5.1, and is found to be $\omega /(2 \pi)=0.17 \mathrm{~Hz}$. In this data, the particle remains in frame until drifting approximately $125 \mu \mathrm{m}$ during the first $4 \mathrm{hrs}$ due to the large tilt drift. The table never returned to the angle necessary for the particle to return to the frame. This behavior is unacceptable for the long-duration experiments we plan to pursue.

The particle displacement during the stabilized tilt measurement is shown by the black curve in Fig. 5.3. The total range of the displacement is $\Delta z \approx 10 \mu \mathrm{m}$, so the particle always remains near the center of the frame. During the four relatively large disturbances in tilt, the displacement also jumps. Besides these four instances, the displacement shows little correlation with the relative tilt, showing that the particle is not limited by the tilt stability. The origin of the particle motion is not clear, but may be due to changes in the charge of the particle over time (likely due to the presence of air in the chamber).

We have shown that with a simple modification of the vibration isolators of an optical table, its tilt can be actively stabilize to an RMS variation of $0.35 \mu \mathrm{rad}$. This is nearly three orders of magnitude less than without stabilization. The need for such stabilization is evident in the displacement measurements of a trapped microsphere on the table. Without stabilization, the particle leaves the frame of the camera; with stabilization, the particle moves by less than one diameter and the particle displacement drift is not limited by the tilt stability.

This work was supported by the NSF under awards PHY-1707789, PHY-1757005, PHY1707678, PHY-1806596, OIA-1458952, and OIA-1003907; a block gift from the II-VI Foundation; NASA under awards ISFM-80NSSC18K0538 and TCAN-80NSSC18K1488; the state 
of West Virginia HEPC; and West Virginia University.

The data that support the findings of this study are available from the corresponding author upon reasonable request. 


\section{Appendix A}

The following pages contain a copy of the SEOBNRv3 documentation Jupyter notebook output as a PDF for easy reading. Note that the notebook has been output in landscape mode and that two pages have been included per page of this dissertation. 


\section{Tutorial-SEOBNR_Documentation}

March 26, 2020

\section{-1 The Spinning Effective One-Body Hamiltonian}

\section{-1.1 Author: Tyler Knowles}

-1.1.1 Formatting improvements courtesy Brandon Clark

-1.2 This module documents the reduced spinning effective one-body Hamiltonian as numerically implemented in LALSuite's SEOBNRv3 gravitational waveform approximant.

Notebook Status: Validated

Validation Notes: This module has been validated against the LALSuite SEOBNRv3/SEOBNRv3_opt code that was reviewed and approved for LIGO parameter estimation by the LIGO Scientific Collaboration. That is, the value $H_{\text {real }}$ output from this notebook agrees to roundoff error with the value of $H_{\text {real }}$ computed by the LALSuite function XLALSimIMRSpinPrecEOBHamiltonian()

\section{-1.3 Introduction}

\section{-1.3.1 The Physical System of Interest}

Consider two black holes with masses $m_{1}, m_{2}$ and spins $\mathbf{S}_{1}, \mathbf{S}_{2}$ in a binary system. The spinning effective one-body ("SEOB") Hamiltonian $H_{\text {real }}$ (defined in Section 2) describes the dynamics of this system; we will define $H_{\text {real }}$ as in Barausse and Buonanno (2010) Section VE. There, $H_{\text {real }}$ is canonically transformed and mapped to an effective Hamiltonian $H_{\text {eff }}$ (defined in Section 3) describing the motion of a test particle of mass $\mu$ (defined in Section 18.4) and spin $\mathbf{S}^{*}$ (defined in Section 10.1) moving in a defomred Kerr background. Here we seek to break up $H_{\text {real }}$ and document the terms in such a way that the resulting Python code can be used to numerically evaluate $H_{\text {real }}$.

We write $H_{\text {real }}$ in terms of Cartesian quasi-isotropic coordinates $x, y$, and $z$ (see Barausse and Buonanno (2010) Section III). The spatial coordinates $r, \theta$, and $\phi$ referenced throughout are Boyer-Lindquist coordinates (see Barausse and Buonanno (2010) Section IV).

Please note that throughout this notebook we adpot the following conventions:

1. $c=1$ where $c$ is the speed of light in a vacuum,

2. spacial tensor indicies are denoted by lowercase Latin letters,

3. repeated indices indicate Einstein summation notation, and

4. we normalize $M=1$ in all terms except for $\eta$ and $\mu$ for agreement with LALSuite. Nonetheless, $M$ appears in other text cells for comparison with the cited literature.

-1.3.2 Citations

Throughout this module, we will refer to * Barausse and Buonanno (2010) as BB2010, * Barausse and Buonanno (2011) as BB2011, * Pan, Buonanno, Buchman, et. al. 2010 as P2010, * Taracchini, Buonanno, Pan, et al (2014) as T2014, * Taracchini, Pan, Buonanno, et al (2012) as T2012, and * Damour, Jaranowski, and Schaefer (2000) as D2000.

\section{Table of Contents}

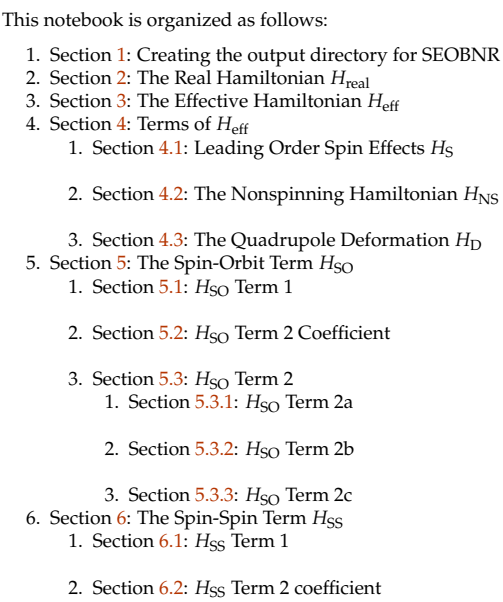




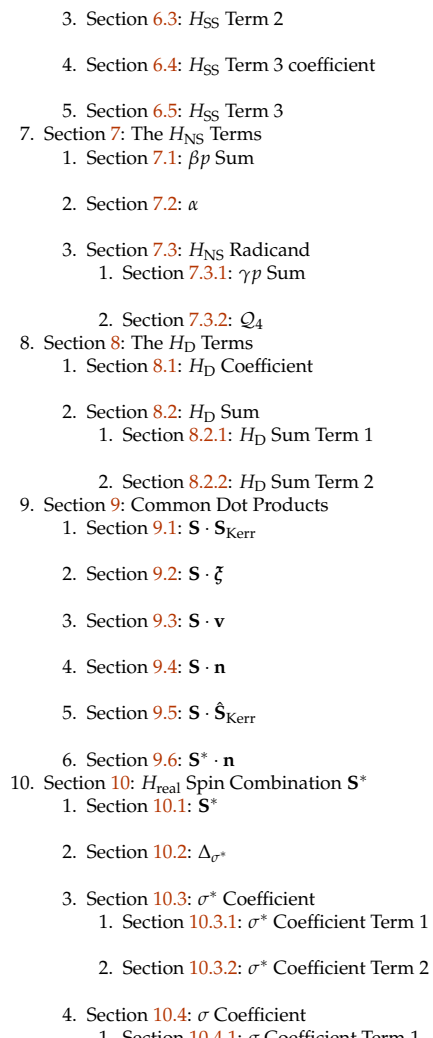

1. Section 10.4.1: $\sigma$ Coefficient Term 1

2. Section 10.4.2: $\sigma$ Coefficient Term 2

3. Section 10.4.3: $\sigma$ Coefficient Term 3

11. Section 11: Derivatives of the Metric Potential

1. Section 11.1: $\omega_{r}$

2. Section 11.2: $v_{r}$

3. Section 11.3: $\mu_{r}$

4. Section 11.4: $\omega_{\cos \theta}$

5. Section 11.5: $v_{\cos \theta}$

6. Section 11.6: $\mu_{\cos \theta}$

7. Section 11.7: $\Lambda_{t}^{\prime}$

8. Section 11.8: $\tilde{\omega}_{\mathrm{fd}}^{\prime}$

12. Section 12. The Deformed and Rescaled Metric Potentials

1. Section 12.1: $\omega$

2. Section 12.2: $e^{2}$

3. Section $12.3: \tilde{B}$

4. Section 12.4: $\tilde{B}_{r}$

5. Section 12.5: $e^{2 \check{\mu}}$

6. Section $12.6: \tilde{J}$

7. Section 12.7: $Q$

1. Section 12.7.1: $\frac{\Delta_{r}}{\Sigma}(\hat{\mathbf{p}} \cdot \mathbf{n})^{2}$

2. Section 12.7.2: Q Coefficient 1

3. Section 12.7.3: Q Coefficient 2

13. Section 13: Tortoise terms

1. Section 13.1: $p_{\phi}$

2. Section 13.2: $\hat{\mathbf{p}} \cdot \mathbf{v} r$

3. Section 13.3: $\hat{\mathbf{p}} \cdot \mathbf{n}$ 


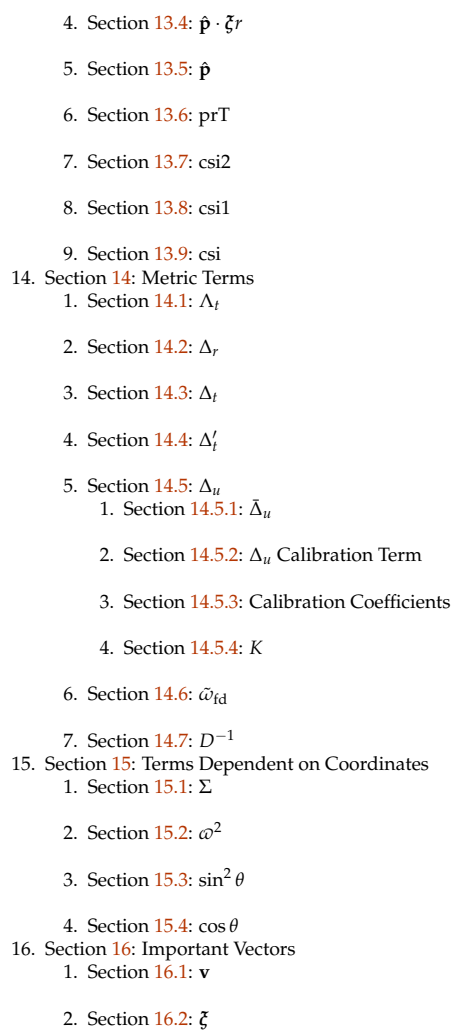

3. Section 16.3: $\mathbf{e}_{3}$

4. Section 16.4: $\mathbf{n}$

17. Section 17: Spin Combinations $\sigma, \sigma^{*}$, and $\mathbf{S}_{\mathrm{Kerr}}$ 1. Section 17.1: $a$

2. Section 17.2: $\hat{\mathbf{S}}_{\text {Kerr }}$

3. Section 17.3: $\left|\mathbf{S}_{\text {Kerr }}\right|$

4. Section 17.4: $\mathbf{S}_{\text {Ker }}$

5. Section 17.5: $\sigma$

6. Section 17.6: $\sigma^{*}$

18. Section 18: Fundamental Quantities

1. Section 18.1: $u$

2. Section 18.2: $r$

3. Section $18.3: \eta$

4. Section 18.4: $\mu$

5. Section $18.5: M$

19. Section 19: Validation

20. Section 20: Output this notebook to LTEX-formatted PDF file

\section{Step 0: Creating the output directory for SEOBNR [Back to Section 0]}

First we create the output directory for SEOBNR (if it does not already exist):

[1]: import cmdline_helper as cmd \#NRPy+: Multi-platform Python command-line interface

\# Create $C$ code output directory:

Ccodesdir = "SEOBNR"

\# Then create an output directory in case it does not exist

cmd.mkdir(Ccodesdir) 


\section{Step 1: The real Hamiltonian $H_{\text {real }}$ [Back to Section 0]}

The SEOB Hamiltonian $H_{\text {real }}$ is given by BB2010 Equation (5.69):

$$
H_{\text {real }}=M \sqrt{1+2 \eta\left(\frac{H_{\text {eff }}}{\mu}-1\right)} .
$$

Here $H_{\text {eff }}$ (defined in Section 3) is an effective Hamiltonian (see Section -1.3) and $M$ (defined in Section 18.5), $\mu$ (defined in Section 18.4), and $\eta$ (defined in Section 18.3) are constants determined by $m_{1}$ and $m_{2}$.

[2]: \%\%ritefile \$Ccodesdir/Hamiltonian-Hreal_on_top.txt

Hreal $=\operatorname{sp} . \operatorname{sqrt}(1+2 * \operatorname{eta} *(\operatorname{Heff}-1))$

Overwriting SEOBNR/Hamiltonian-Hreal_on_top.txt

\section{Step 2: The Effective Hamiltonian $H_{\text {eff }}$ [Back to Section 0]}

The effective Hamiltonian $H_{\text {eff }}$ is given by BB2010 Equation (5.70):

$$
H_{\mathrm{eff}}=H_{\mathrm{S}}+\underbrace{\beta^{i} p_{i}+\alpha \sqrt{\mu^{2}+\gamma^{i j} p_{i} p_{j}+\mathcal{Q}_{4}}}_{H_{\mathrm{NS}}}-\underbrace{\frac{\mu}{2 M r^{3}}\left(\delta^{i j}-3 n^{i} n^{j}\right) S_{i}^{*} S_{j}^{*}}_{H_{\mathrm{D}}} .
$$

Here $H_{\mathrm{S}}$ (considered further in Section 4.1) denotes leading order effects of spin-spin and spin-orbit coupling, $H_{\mathrm{NS}}$ (considered further in Section 4.2) is the Hamiltonian for a nonspinning test particle, and $H_{\mathrm{D}}$ (considered further in Section 4.3) describes quadrupole deformation of the coupling of the particle's spin with itself to leading order. T2014 adds to $H_{\text {eff }}$ a $3 \mathrm{PN}$ spin-spin term given by

$$
\frac{d_{\mathrm{SS} \eta}}{r^{4}}\left(\mathbf{S}_{1}^{2}+\mathbf{S}_{2}^{2}\right)
$$

where $d_{\mathrm{SS}}$ is an adjustable parameter determined by fitting to numerical relativity results. We take $u \equiv \frac{1}{r}$ (as described in Section 18.1) and define $\eta$ in Section 18.3 .

[3]: \%\%rritefile -a \$Ccodesdir/Hamiltonian-Hreal_on_top.txt

Heff $=$ Hs + Hns - Hd + dSS $*$ eta $* u * u * u * u *(S 1 x * S 1 x+S 1 y * S 1 y+S 1 z * S 1 z+S 2 x * S 2 x+S 2 y * S 2 y+S 2 z * S 2 z)$ $\mathrm{dSS}=8.127-154.2 *$ eta $+830.8 *$ eta $*$ eta

Appending to SEOBNR/Hamiltonian-Hreal_on_top.txt

\section{Step 3: Terms of $H_{\text {eff }}$ [Back to Section 0]}

In this step, we break down each of the terms $H_{\mathrm{S}}$ (defined in Section 4.1), $H_{\mathrm{NS}}$ (defined in Section 4.2), and $H_{\mathrm{D}}$ (defined in Section 4.3) in $H_{\text {eff }}$ (defined in Section 3).

4.1 Step 3.a: Leading Order Spin Effects $H_{\mathrm{S}}$ [Back to Section 0]

From BB2010 Equation (4.17),

$$
H_{\mathrm{S}}=H_{\mathrm{SO}}+H_{\mathrm{SS}}
$$

where $H_{\mathrm{SO}}$ (defined in Section 5) includes spin-orbit terms and $H_{\mathrm{SS}}$ (defined in Section 6) includes spin-spin terms.

[4]: \%\%ritefile -a \$Ccodesdir/Hamiltonian-Hreal_on_top.txt

$\mathrm{Hs}=\mathrm{HsO}+\mathrm{Hss}$

Appending to SEOBNR/Hamiltonian-Hreal_on_top.txt

4.2 Step 3.b: The Nonspinning Hamiltonian $H_{\mathrm{NS}}$ [Back to Section 0]

We defined $H_{\mathrm{NS}}$ in Section 3 as

$$
H_{\mathrm{NS}}=\underbrace{\beta^{i} p_{i}}_{\beta p \text { sum }}+\alpha \sqrt{\underbrace{\mu^{2}+\gamma^{i j} p_{i} p_{j}+\mathcal{Q}_{4}}_{H_{\mathrm{NS}} \text { radicand }}} .
$$

We compute $\beta p$ sum in Section 7.1, $\alpha$ in Section 7.2, and $H_{\mathrm{NS}}$ radicand in Section 7.3.

[5]: \%\%writefile -a \$Ccodesdir/Hamiltonian-Hreal_on_top.txt

Hns $=$ betapsum + alpha*sp.sqrt(Hnsradicand $)$

Appending to SEOBNR/Hamiltonian-Hreal_on_top.txt 
We defined $H_{\mathrm{D}}$ in Section 3 as:

$$
H_{\mathrm{D}}=\underbrace{\frac{\mu}{2 M r^{3}}}_{H_{\mathrm{D}} \text { coefficient }} \underbrace{\left(\delta^{i j}-3 n^{i} n^{j}\right) S_{i}^{*} S_{j}^{*}}_{H_{\mathrm{D}} \text { sum }}
$$

We compute $H_{\mathrm{D}}$ coefficient in Section 8.1 and $H_{\mathrm{D}}$ sum in Section 8.2.

[6]: \%\%writefile -a \$Ccodesdir/Hamiltonian-Hreal_on_top.txt

Hd $=$ Hdcoeff $*$ Hdsum

Appending to SEOBNR/Hamiltonian-Hreal_on_top.txt

\section{Step 4: The Spin-Orbit Term $H_{\mathrm{SO}}$ [Back to Section 0]}

We will write BB2010 Equation (4.18) as:

$$
H_{\text {SO }}=H_{\text {SO }} \text { Term } 1+H_{\text {SO }} \text { Term } 2 \text { coefficient } * H_{\text {SO }} \text { Term } 2 .
$$

We define and consider $H_{\mathrm{SO}}$ Term 1 in Section 5.1, $H_{\mathrm{SO}}$ Term 2 coefficient in Section 5.2, and $H_{\mathrm{SO}}$ Term 2 in Section 5.3 .

[7]: \%\%writefile -a \$Ccodesdir/Hamiltonian-Hreal_on_top.txt

Hso $=$ HsoTerm1 + HsoTerm2coeff $*$ HsoTerm2

Appending to SEOBNR/Hamiltonian-Hreal_on_top.txt

5.1 Step 4.a: $H_{\text {SO }}$ Term 1 [Back to Section 0]

Combining our notation $H_{\text {SO }}$ (defined in Section 5) with BB2010 Equation (4.18), we have

$$
H_{\mathrm{SO}} \operatorname{Term} 1=\frac{e^{2 v-\tilde{\mu}}\left(e^{\tilde{\mu}+v}-\tilde{B}\right)(\hat{\mathbf{p}} \cdot \xi r)\left(\mathbf{S} \cdot \hat{\mathbf{S}}_{\mathrm{Kerr}}\right)}{\tilde{B}^{2} \sqrt{\mathrm{Q}} \xi^{2}} .
$$

We will write

$$
H_{\mathrm{SO}} \operatorname{Term} 1=\frac{e^{2 v}\left(e^{\tilde{\mu}} e^{v}-\tilde{B}\right)(\hat{\mathbf{p}} \cdot \xi r)\left(\mathbf{S} \cdot \hat{\mathbf{S}}_{\mathrm{Kerr}}\right)}{e^{\tilde{\mu}} \tilde{B}{ }^{2} \sqrt{Q} \xi^{2}}
$$

We define $e^{\bar{u}}$ in Section 12.5, $e^{v}$ in Section 12.2, $\tilde{B}$ in Section 12.3, $\hat{\mathbf{p}} \cdot \xi r$ in Section 13.4, S $\cdot \hat{\mathbf{S}}_{\text {Kerr }}$ in Section 9.5, $Q$ in Section 12.7, and $\xi^{2}$ in Section 15.3.

[8]: \%\%writefile -a \$Ccodesdir/Hamiltonian-Hreal_on_top.txt

HsoTerm1 $=\exp 2 n u *($ expmu*expnu - Btilde $) * \operatorname{pdotxir} *$ SdotSkerrhat $/(\operatorname{expmu} *$ Btilde $*$ Btilde $*$ sp.sqrt $(\mathrm{Q}) * x i s q)$

Appending to SEOBNR/Hamiltonian-Hreal_on_top.tx

5.2 Step 4.b: $H_{\text {SO }}$ Term 2 Coefficient [Back to Section 0$]$

Combining our notation $H_{\mathrm{SO}}$ (defined in Section 5) with BB2010 Equation (4.18), we have

$$
H_{\text {SO }} \text { Term } 2 \text { coefficient }=\frac{e^{v-2 \tilde{\mu}}}{\tilde{B}^{2}(\sqrt{\bar{Q}}+1) \sqrt{\bar{Q} \xi^{2}}}
$$

which we write in the form

$$
H_{\text {SO }} \text { Term } 2 \text { coefficient }=\frac{e^{v}}{e^{2 \tilde{\mu}} \tilde{B}^{2}(Q+\sqrt{Q}) \tilde{\zeta}^{2}} .
$$

We define and consider $e^{v}$ in Section 12.2, $e^{\tilde{\mu}}$ in Section 12.5, $\tilde{B}$ in Section 12.3, $Q$ in Section 12.7, and $\tilde{\xi}^{2}$ in Section 15.3 .

[9]: \% \%ritefile -a \$Ccodesdir/Hamiltonian-Hreal_on_top.txt

HsoTerm2coeff $=\operatorname{expnu} /(\exp 2 m u * B t i l d e * B t i l d e *(Q+\operatorname{sp} . \operatorname{sqrt}(Q)) * x i s q)$

Appending to SEOBNR/Hamiltonian-Hreal_on_top.txt 
Combining our notation $H_{\mathrm{SO}}$ (defined in Section 5) with BB2010 Equation (4.18), we have

$$
\begin{aligned}
H_{\mathrm{SO}} \operatorname{Term} 2= & \underbrace{(\mathbf{S} \cdot \boldsymbol{\xi}) \tilde{J}\left[\mu_{r}(\hat{\mathbf{p}} \cdot \mathbf{v} r)(\sqrt{Q}+1)-\mu_{\cos \theta}(\hat{\mathbf{p}} \cdot \mathbf{n}) \tilde{\xi}^{2}-\sqrt{Q}\left(v_{r}(\hat{\mathbf{p}} \cdot \mathbf{v} r)+\left(\mu_{\cos \theta}-v_{\cos \theta}\right)(\hat{\mathbf{p}} \cdot \mathbf{n}) \tilde{\xi}^{2}\right)\right] \tilde{B}^{2}}_{H_{\mathrm{SO}} \operatorname{Term} 2 \mathrm{a}} \\
& +\underbrace{e^{\tilde{\mu}+v}(\hat{\mathbf{p}} \cdot \boldsymbol{\xi} r)(2 \sqrt{Q}+1)\left[\tilde{J} v_{r}(\mathbf{S} \cdot \mathbf{v})-v_{\cos \theta}(\mathbf{S} \cdot \mathbf{n}) \tilde{\zeta}^{2}\right]}_{H_{\mathrm{SO}} \text { Term 2b }} \tilde{B}-\underbrace{\tilde{J} \tilde{B}_{r} e^{\tilde{\mu}+v}\left(\hat{\mathbf{p}} \cdot \boldsymbol{\xi}_{r}\right)(\sqrt{\bar{Q}}+1)(\mathbf{S} \cdot \mathbf{v})}_{H_{\mathrm{SO}} \text { Term 2c }}
\end{aligned}
$$

We compute $H_{\mathrm{SO}}$ Term 2a in Section 5.3.1, $H_{\mathrm{SO}}$ Term $2 \mathrm{~b}$ in Section 5.3.2, and $H_{\mathrm{SO}}$ Term 2c in Section 5.3.3.

[10]: \%\%writefile -a \$Ccodesdir/Hamiltonian-Hreal_on_top.txt

HsoTerm2 $=$ HsoTerm2a + HsoTerm2b - HsoTerm2c

Appending to SEOBNR/Hamiltonian-Hreal_on_top.txt

5.3.1 Step 4.c.i: $H_{\mathrm{SO}}$ Term 2a [Back to Section 0]

We defined $H_{\mathrm{S} 0}$ Term 2a in Section 5.3 as

$$
H_{\mathrm{SO}} \operatorname{Term} 2 \mathrm{a}=(\mathbf{S} \cdot \xi) \tilde{J}\left[\mu_{r}(\hat{\mathbf{p}} \cdot \mathbf{v} r)(\sqrt{\bar{Q}}+1)-\mu_{\cos \theta}(\hat{\mathbf{p}} \cdot \mathbf{n}) \tilde{\zeta}^{2}-\sqrt{Q}\left(v_{r}(\hat{\mathbf{p}} \cdot \mathbf{v} r)+\left(\mu_{\cos \theta}-v_{\cos \theta}\right)(\hat{\mathbf{p}} \cdot \mathbf{n}) \xi^{2}\right)\right] \tilde{B}^{2}
$$

We define $\mathbf{S} \cdot \xi$ in Section 9.2, $\tilde{J}$ in Section 12.6, $\mu_{r}$ in Section 11.3, $\hat{\mathbf{p}} \cdot \mathbf{v} r$ in Section 13.2, $Q$ in Section 12.7, $\mu_{\cos \theta}$ in Section 11.6, $\hat{\mathbf{p}} \cdot \mathbf{n}$ in Section 13.3, $\tilde{\xi}^{2}$ in Section 15.3, $v_{r}$ in Section 11.2, $v_{\cos \theta}$ in Section 11.5, and $\tilde{B}$ in Section 12.3.

[11]: \%)writefile -a \$Ccodesdir/Hamiltonian-Hreal_on_top.txt

HsoTerm2a $=$ Sdotxi $*$ Jtilde $*(\operatorname{mur} *$ pdotvr $*(\operatorname{sp} . \operatorname{sqrt}(\mathrm{Q})+1)-\operatorname{mucostheta} *$ pdotn $*$ xisq

- $\operatorname{sp} . \operatorname{sqrt}(\mathrm{Q}) *($ nur $*$ pdotvr $+($ mucostheta - nucostheta $) *$ pdotn $* \mathrm{xisq})) *$ Btilde $*$ Btilde

Appending to SEOBNR/Hamiltonian-Hreal_on_top.txt

5.3.2 Step 4.c.ii: $H_{\mathrm{SO}}$ Term $\mathbf{2 b}$ [Back to Section 0$]$

We defined $H_{\mathrm{S} 0}$ Term $2 \mathrm{~b}$ in Section 5.3 as

$$
H_{\mathrm{SO}} \operatorname{Term} 2 \mathrm{~b}=e^{\tilde{\mu}+v}\left(\hat{\mathbf{p}} \cdot \xi^{r}\right)(2 \sqrt{Q}+1)\left[\tilde{f} v_{r}(\mathbf{S} \cdot \mathbf{v})-v_{\cos \theta}(\mathbf{S} \cdot \mathbf{n}) \tilde{\zeta}^{2}\right] \tilde{B} .
$$

We define $e^{\tilde{\mu}}$ in Section 12.5, $e^{v}$ in Section 12.2, $\hat{\mathbf{p}} \cdot \xi r$ in Section 13.4, $Q$ in Section 12.7, $\tilde{J}$ in Section 12.6, $v_{r}$ in Section 11.2, S $\cdot \mathbf{v}$ in Section 9.3, $v_{\cos \theta}$ in Section $11.5, \mathbf{S} \cdot \mathbf{n}$ in Section 9.4, $\tilde{\zeta}^{2}$ in Section 15.3, and $\widetilde{B}$ in Section 12.3 .

[12]: \% \%ritefile -a \$Ccodesdir/Hamiltonian-Hreal_on_top.txt

HsoTerm2b $=$ expmu*expnu $*$ pdotxir $*(2 * \operatorname{sp} . \operatorname{sqrt}(Q)+1) *($ Jtilde $*$ nur $* \operatorname{Sdotv}-$ nucostheta $* \operatorname{Sdotn} *$ Xisq $) *$ Btilde

Appending to SEOBNR/Hamiltonian-Hreal_on_top.txt

5.3.3 Step 4.c.iii: $H_{\mathrm{SO}}$ Term 2c [Back to Section 0]

We defined $H_{\mathrm{SO}}$ Term 2c in Section 5.3 as

$$
H_{\mathrm{SO}} \operatorname{Term} 2 \mathrm{c}=\tilde{J} \tilde{B}_{r} e^{\bar{\mu}+v}\left(\hat{\mathbf{p}} \cdot \xi^{r}\right)(\sqrt{\bar{Q}}+1)(\mathbf{S} \cdot \mathbf{v})
$$

We define $\tilde{J}$ in Section 12.6, $\tilde{B}_{r}$ in Section 12.4, $e^{\tilde{\mu}}$ in Section 12.5, $e^{v}$ in Section 12.2, $\hat{\mathbf{p}} \cdot \tilde{\xi} r$ in Section 13.4, Q in Section 12.7, and $\mathbf{S} \cdot \mathbf{v}$ in Section 9.3.

[13]: \%\%ritefile -a \$Ccodesdir/Hamiltonian-Hreal_on_top.txt

HsoTerm2c $=$ Jtilde $*$ Brtilde $*$ expmu*expnu $*$ pdotxir $*(\mathbf{s p} . \operatorname{sqrt}(\mathbf{Q})+1) * \operatorname{Sdotv}$

Appending to SEOBNR/Hamiltonian-Hreal_on_top.txt

\section{Step 5: The Spin-Spin Term $H_{\mathrm{SS}}$ [Back to Section 0]}

We will write BB2010 Equation (4.19) as

$H_{\mathrm{SS}}=H_{\mathrm{SS}}$ Term $1+H_{\mathrm{SS}}$ Term 2 coefficient $* H_{\mathrm{SS}}$ Term $2+H_{\mathrm{SS}}$ Term 3 coefficient $* H_{\mathrm{SS}}$ Term 3. 
We define $H_{\mathrm{SS}}$ Term 1 in Section 6.1, $H_{\mathrm{SS}}$ Term 2 coefficient in Section 6.2, $H_{\mathrm{SS}}$ Term 2 in Section 6.3, $H_{\mathrm{SS}}$ Term 3 coefficient in Section 6.4 , and $H_{\mathrm{SS}}$ Term 3 in Section 6.5 .

[14]: \%\%ritefile -a \$Ccodesdir/Hamiltonian-Hreal_on_top.txt

Hss $=$ HssTerm1 + HssTerm2Coeff $*$ HssTerm2 + HssTerm3coeff $*$ HssTerm3

Appending to SEOBNR/Hamiltonian-Hreal_on_top.txt

6.1 Step 5.a: $H_{\mathrm{SS}}$ Term 1 [Back to Section 0]

Combining BB2010 Equation (4.19) with our definition of $H_{S S}$ Term 1 in Section 6, we have

$$
H_{\mathrm{SS}} \operatorname{Term} 1=\omega\left(\mathbf{S} \cdot \hat{\mathbf{S}}_{\mathrm{Kerr}}\right) .
$$

We define $\omega$ in Section 12.1 and $\mathbf{S} \cdot \hat{\mathbf{S}}_{\text {Kerr }}$ in Section 9.5.

[15]: \%\%writefile -a \$Ccodesdir/Hamiltonian-Hreal_on_top.txt

HssTerm1 = omega $*$ SdotSkerrhat

Appending to SEOBNR/Hamiltonian-Hreal_on_top.txt

\subsection{Step 5.b: $H_{\text {SS }}$ Term 2 Coefficient [Back to Section 0]}

Combining BB2010 Equation (4.19) with ore definition of $H_{\mathrm{SS}}$ Term 2 coefficient in Section 6, we have

$$
H_{\mathrm{SS}} \text { Term } 2 \text { coefficient }=\frac{e^{-3 \tilde{\mu}-v} \tilde{\omega} \omega_{r}}{2 \tilde{B}(\sqrt{\bar{Q}}+1) \sqrt{\bar{Q} \xi^{2}}}
$$

which we write as

$$
H_{\mathrm{SS}} \text { Term } 2 \text { coefficient }=\frac{\tilde{J} \omega_{r}}{2 e^{2 \tilde{\mu}} e^{\tilde{\mu}} e^{v} \tilde{B}(Q+\sqrt{Q}) \tilde{\zeta}^{2}} .
$$

We define $\tilde{J}$ in Section 12.6, $\omega_{r}$ in Section 11.1, $e^{\tilde{\mu}}$ in Section 12.5, $e^{v}$ in Section 12.2, $\tilde{B}$ in Section 12.3, $Q$ in Section 12.7, and $\tilde{\zeta}^{2}$ in Section 15.3.

[16]: \%\%writefile -a \$Ccodesdir/Hamiltonian-Hreal_on_top.txt

HssTerm2coeff $=$ Jtilde $*$ omegar $/(2 * \exp 2 m u * \operatorname{expmu} * \operatorname{expnu} * \operatorname{Btilde} *(Q+\operatorname{sp} \cdot \operatorname{sqrt}(Q)) * x i s q)$

Appending to SEOBNR/Hamiltonian-Hreal_on_top.txt

6.3 Step 5.c: $H_{\mathrm{SS}}$ Term 2 [Back to Section 0]

Combining BB2010 Equation (4.19) with our definition of $H_{\mathrm{SS}}$ Term 2 in Section 6, we have

$$
H_{\mathrm{SS}} \operatorname{Term} 2=-e^{\tilde{\mu}+v}(\hat{\mathbf{p}} \cdot \mathbf{v} r)(\hat{\mathbf{p}} \cdot, r)(\mathbf{S} \cdot,) \tilde{B}+e^{2(\tilde{\mu}+v)}(\hat{\mathbf{p}} \cdot, r)^{2}(\mathbf{S} \cdot \mathbf{v})+e^{2 \tilde{\mu}}(1+\sqrt{Q}) \sqrt{Q}(\mathbf{S} \cdot \mathbf{v}) \tilde{\xi}^{2} \tilde{B}^{2}+\tilde{J}(\hat{\mathbf{p}} \cdot \mathbf{n})[(\hat{\mathbf{p}} \cdot \mathbf{v} r)(\mathbf{S} \cdot \mathbf{n})-\tilde{J}(\hat{\mathbf{p}} \cdot \mathbf{n})(\mathbf{S} \cdot \mathbf{v})] \xi^{2} \tilde{B}^{2}
$$

which we write as

$$
\begin{aligned}
H_{\mathrm{SS}} \operatorname{Term} 2= & e^{\tilde{\mu}}(\hat{\mathbf{p}} \cdot, r)\left[e^{\tilde{\mu}} e^{2 v}(\hat{\mathbf{p}} \cdot, r)(\mathbf{S} \cdot \mathbf{v})-e^{v}(\hat{\mathbf{p}} \cdot \mathbf{v} r)(\mathbf{S} \cdot,) \tilde{B}\right] \\
& +\tilde{\zeta}^{2} \tilde{B}^{2}\left\{e^{2 \tilde{\mu}}(\sqrt{Q}+Q)(\mathbf{S} \cdot \mathbf{v})+\tilde{J}(\hat{\mathbf{p}} \cdot \mathbf{n})[(\hat{\mathbf{p}} \cdot \mathbf{v} r)(\mathbf{S} \cdot \mathbf{n})-\tilde{J}(\hat{\mathbf{p}} \cdot \mathbf{n})(\mathbf{S} \cdot \mathbf{v})]\right\}
\end{aligned}
$$

We define $e^{\tilde{\mu}}$ in Section 12.5, $\hat{\mathbf{p}} \cdot \xi r$ in Section 13.4, $e^{v}$ in Section 12.2, $\mathbf{S} \cdot \mathbf{v}$ in Section 9.3, $\hat{\mathbf{p}} \cdot \mathbf{v} r$ in Section 13.2, $\mathbf{S} \cdot \boldsymbol{\xi}$ in Section 9.2, $\tilde{B}$ in Section $12.3, Q$ in Section $12.7, \tilde{J}$ in Section 12.6, $\hat{\mathbf{p}} \cdot \mathbf{n}$ in Section 13.3, $\mathbf{S} \cdot \mathbf{n}$ in Section 9.4, and $\tilde{\zeta}^{2}$ in Section 15.3.

[17]: \%\%ritefile -a \$Ccodesdir/Hamiltonian-Hreal_on_top.txt

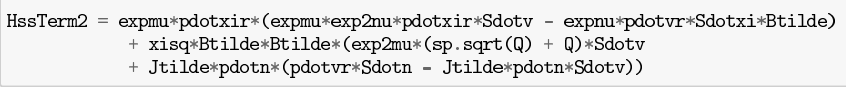

Appending to SEOBNR/Hamiltonian-Hreal_on_top.txt

\subsection{Step 5.d: $H_{\mathrm{SS}}$ Term 3 Coefficient [Back to Section 0]}

Combining BB2010 Equation (4.19) with our definition of $H_{\mathrm{SS}}$ Term 3 coefficient in Section 6, we have

$$
H_{\mathrm{SS}} \text { Term } 3 \text { coefficient }=\frac{e^{-3 \tilde{\mu}-v} \omega_{\cos \theta}}{2 \tilde{B}(\sqrt{Q}+1) \sqrt{Q}}
$$


which we write as

$$
H_{\mathrm{SS}} \text { Term } 3 \text { coefficient }=\frac{\omega_{\cos \theta}}{2 e^{2 \tilde{\mu}} e^{\tilde{\mu}} e^{v} \tilde{B}(Q+\sqrt{\bar{Q}})} .
$$

We define $\omega_{\cos \theta}$ in Section 11.4, $e^{\tilde{\mu}}$ in Section 12.5, $e^{v}$ in Section 12.2, and $\tilde{B}$ in Section 12.3, $Q$ in Section 12.7.

[18]: \%\%rritefile -a \$Ccodesdir/Hamiltonian-Hreal_on_top.txt

HssTerm3coeff $=$ omegacostheta $/(2 * \exp 2 m u * \operatorname{expmu} * \operatorname{expnu} * \mathrm{Btilde} *(\mathrm{Q}+\operatorname{sp} \cdot \operatorname{sqrt}(\mathrm{Q})))$

Appending to SEOBNR/Hamiltonian-Hreal_on_top.txt

6.5 Step 5.e: $H_{\mathrm{SS}}$ Term 3 [Back to Section 0]

Combining BB2010 Equation (4.19) with our definition of $H_{\mathrm{SS}}$ Term 3 in Section 6, we have

$$
\begin{aligned}
H_{\mathrm{SS}} \text { Term } 3= & -e^{2(\tilde{\mu}+v)}(\hat{\mathbf{p}} \cdot, r)^{2}(\mathbf{S} \cdot \mathbf{n})+e^{\tilde{\mu}+v} \tilde{J}(\hat{\mathbf{p}} \cdot \mathbf{n})(\hat{\mathbf{p}} \cdot, r)(\mathbf{S} \cdot,) \tilde{B} \\
& +\left[(\mathbf{S} \cdot \mathbf{n})(\hat{\mathbf{p}} \cdot \mathbf{v} r)^{2}-\tilde{J}(\hat{\mathbf{p}} \cdot \mathbf{n})(\mathbf{S} \cdot \mathbf{v})(\hat{\mathbf{p}} \cdot \mathbf{v} r)-e^{2 \tilde{\mu}}(1+\sqrt{Q}) \sqrt{Q}(\mathbf{S} \cdot \mathbf{n}) \tilde{\zeta}^{2}\right] \tilde{B}^{2}
\end{aligned}
$$

which we write as

$$
\begin{aligned}
H_{\mathrm{SS}} \text { Term } 3= & e^{\tilde{\mu}} e^{v}(\hat{\mathbf{p}} \cdot, r)\left[\tilde{J}(\hat{\mathbf{p}} \cdot \mathbf{n})\left(\mathbf{S} \cdot{ }_{,}\right) \tilde{B}-e^{\tilde{u}} e^{v}(\hat{\mathbf{p}} \cdot, r)(\mathbf{S} \cdot \mathbf{n})\right] \\
& +\left\{(\hat{\mathbf{p}} \cdot \mathbf{v} r)[(\mathbf{S} \cdot \mathbf{n})(\hat{\mathbf{p}} \cdot \mathbf{v} r)-\tilde{J}(\hat{\mathbf{p}} \cdot \mathbf{n})(\mathbf{S} \cdot \mathbf{v})]-e^{2 \tilde{\mu}}(\sqrt{Q}+Q)(\mathbf{S} \cdot \mathbf{n}) \tilde{\xi}^{2}\right\} \tilde{B}^{2}
\end{aligned}
$$

We define $e^{\tilde{\mu}}$ in Section 12.5, $e^{v}$ in Section 12.2, $\hat{\mathbf{p}} \cdot \boldsymbol{\xi} r$ in Section 13.4, $\tilde{J}$ in Section 12.6, $\hat{\mathbf{p}} \cdot \mathbf{n}$ in Section 13.3, $\mathbf{S} \cdot \boldsymbol{\xi}$ in Section 9.2, $\tilde{B}$ in Section 12.3, $\mathbf{S} \cdot \mathbf{n}$ in Section 9.4 $\hat{\mathbf{p}} \cdot \mathbf{v} r$ in Section 13.2, $\mathbf{S} \cdot \mathbf{v}$ in Section 9.3, $Q$ in Section 12.7, and $\tilde{\xi}^{2}$ in Section 15.3.

[19]: \%\%writefile -a \$Ccodesdir/Hamiltonian-Hreal_on_top.txt

HssTerm3 $=$ expmu $*$ expnu $*$ pdotxir $*(J t i l d e *$ pdotn $*$ Sdotxi $*$ Btilde - expmu*expnu $*$ pdotxir $*$ Sdotn $)$

$+($ pdotvr $*($ Sdotn $*$ pdotvr - Jtilde*pdotn $*$ Sdotv $)-\exp 2 m u *(\operatorname{sp} . \operatorname{sqrt}(\mathrm{Q})+\mathrm{Q}) * \operatorname{Sdotn} * \mathrm{xisq}) *$ Btilde $*$ Btilde

Appending to SEOBNR/Hamiltonian-Hreal_on_top.txt

\section{Step 6: $H_{\mathrm{NS}}$ Terms [Back to Section 0]}

We collect here the terms in $H_{\mathrm{NS}}$ (defined in Section 4.2).

7.1 Step 6.a: $\beta p$ sum [Back to Section 0]

We defined the term $\beta p$ sum in Section 4.2 as

$\beta p$ sum $=\beta^{i} p_{i}$

From BB2010 Equation (5.45), we have

$$
\beta^{i}=\frac{g^{t i}}{g^{t t}}
$$

but from BB2010 Equations (5.36) we see that $g^{t r}=g^{t \theta}=0$. Thus only $\beta^{\phi}$ is nonzero. Combining BB2010 Equations (5.45), (5.36e), and (5.36a), we find

$$
\beta^{\phi}=\frac{-\frac{\tilde{\omega}_{\mathrm{fd}}}{\Delta_{t} \Sigma}}{-\frac{\Lambda_{t}}{\Delta_{t}{ }^{2}}}=\frac{\tilde{\omega}_{\mathrm{fd}}}{\Lambda_{t}}
$$

Therefore

$$
\beta^{i} p_{i}=\frac{\tilde{\omega}_{\mathrm{fd}}}{\Lambda_{t}} p_{\phi}
$$

We define $\tilde{\omega}_{\mathrm{fd}}$ in Section 14.6, $\Lambda_{t}$ in Section 14.1, and $p_{\phi}$ in Section 13.1.

[20]: \%\%rritefile -a \$Ccodesdir/Hamiltonian-Hreal_on_top.txt

betapsum $=$ omegatilde*pphi/Lambdat

Appending to SEOBNR/Hamiltonian-Hreal_on_top.txt 
From BB2010 Equation (5.44), we have

$$
\alpha=\frac{1}{\sqrt{-g^{t t}}}
$$

and from BB2010 Equation (5.36a) we have

$$
g^{t t}=-\frac{\Lambda_{t}}{\Delta_{t} \Sigma}
$$

Therefore

$$
\alpha=\sqrt{\frac{\Delta_{t} \Sigma}{\Lambda_{t}}}
$$

We define $\Delta_{t}$ in Section 14.3, $\Sigma$ in Section 15.1, and $\Lambda_{t}$ in Section 14.1

[21]: \%\%ritefile -a \$Ccodesdir/Hamiltonian-Hreal_on_top.txt

alpha $=$ sp.sqrt (Deltat $*$ Sigma $/$ Lambdat $)$

Appending to SEOBNR/Hamiltonian-Hreal_on_top.txt

\subsection{Step 6.c: $H_{\mathrm{NS}}$ radicand [Back to Section 0]}

Recall that we defined $H_{\text {NS }}$ radicand in Section 4.2 as

$$
H_{\mathrm{NS}} \text { radicand }=\mu^{2}+\underbrace{\gamma^{i j} p_{i} p_{j}}_{\gamma p \text { sum }}+\mathcal{Q}_{4}
$$

We define $\mu$ in Section 18.4, $\gamma p$ sum in Section 7.3.1, and $\mathcal{Q}_{4}$ in Section 7.3.2.

[22]: \%\%writefile -a \$Ccodesdir/Hamiltonian-Hreal_on_top.txt

Hnsradicand $=1+$ gammappsum + Q4

Appending to SEOBNR/Hamiltonian-Hreal_on_top.txt

7.3.1 Step 6.c.i: $\gamma^{i j} p_{i} p_{j}$ [Back to Section 0$]$

From BB2010 Equation (5.46), we have

$$
\gamma^{i j}=g^{i j}-\frac{g^{t i} g^{t j}}{g^{t t}}
$$

Combining this result with BB2010 Equations 5.36, we have

$$
\gamma^{r \theta}=\gamma^{r \phi}=\gamma^{\theta r}=\gamma^{\theta \phi}=\gamma^{\phi r}=\gamma^{\phi \theta}=0
$$

and

$$
\begin{gathered}
\gamma^{r r}=g^{r r}=\frac{\Delta_{r}}{\Sigma} \\
\gamma^{\theta \theta}=g^{\theta \theta}=\frac{1}{\Sigma} \\
\gamma^{\phi \phi}=\frac{\Sigma}{\Lambda_{t} \sin ^{2} \theta} .
\end{gathered}
$$

Therefore

$$
\begin{aligned}
\gamma^{i j} p_{i} p_{j} & =\gamma^{r r} p_{r} p_{r}+\gamma^{\theta \theta} p_{\theta} p_{\theta}+\gamma^{\phi \phi} p_{\phi} p_{\phi} \\
& =\frac{\Delta_{r}}{\Sigma} p_{r}^{2}+\frac{1}{\Sigma} p_{\theta}^{2}+\frac{\Sigma}{\Lambda_{t} \sin ^{2} \theta} p_{\phi}^{2} .
\end{aligned}
$$

Converting Boyer-Lindquist coordinates to tortoise coordinates (the transformation for which is found in the Appendix of P2010), we have

$$
\begin{aligned}
p_{r} & =\hat{\mathbf{p}} \cdot \mathbf{n} \\
p_{\theta} & =\hat{\mathbf{p}} \cdot \mathbf{v} \frac{r}{\sin \theta} \\
p_{\phi} & =\hat{\mathbf{p}} \cdot \xi^{r} .
\end{aligned}
$$

Therefore

$$
\gamma^{i j} p_{i} p_{j}=\frac{\Delta_{r}}{\Sigma}(\hat{\mathbf{p}} \cdot \mathbf{n})^{2}+\Sigma^{-1}\left(\hat{\mathbf{p}} \cdot \mathbf{v} \frac{r}{\sin \theta}\right)^{2}+\frac{\Sigma}{\Lambda_{t} \sin ^{2} \theta}(\hat{\mathbf{p}} \cdot \boldsymbol{\xi} r)^{2} .
$$

We define $\Delta_{r}$ in Section 14.2, $\Sigma$ in Section 17.5, $\hat{\mathbf{p}} \cdot \mathbf{n}$ in Section 13.3, $\hat{\mathbf{p}} \cdot \mathbf{v} r$ in Section 13.2, $\sin ^{2} \theta$ in Section 15.3, $\Lambda_{t}$ in Section 14.1, and $\hat{\mathbf{p}} \cdot \xi r$ in Section 13.4. 
[23]: \%\%writefile -a \$Ccodesdir/Hamiltonian-Hreal_on_top.txt

garmappsum $=$ Deltar $/$ Sigma $*$ pdotn $*$ pdotn $+1 /$ Sigma $*$ pdotvr $*$ pdotvr $/$ sin2theta + Sigma/Lambdat $/$ sin2theta*pdotxir $*$ pdotxir

Appending to SEOBNR/Hamiltonian-Hreal_on_top.txt

7.3.2 Step 6.c.ii: $\mathcal{Q}_{4}[$ Back to Section 0]

From T2012 Equation (15),

$$
\mathcal{Q}_{4} \propto \frac{p_{r^{*}}^{4}}{r^{2}}\left(r^{2}+\chi_{\text {Kerr }}^{2}\right)^{4}
$$

We denote $p_{r^{*}}$ by prT. Converting from tortoise coordinates to physical coordinates(the transformation for which is found in the Appendix of P2010), we find

$$
\mathcal{Q}_{4}=\frac{p r T^{4}}{r^{2}} z_{3}
$$

where $z_{3}$ is found in D2000 Equation (4.34)

$$
z_{3}=2(4-3 v) v
$$

In the notation of BB2010, $v=\eta$ (see discussion after T2012 Equation (2)). Thus

$$
\mathcal{Q}_{4}=2 p r T^{4} u^{2}(4-3 \eta) \eta \text {. }
$$

We define prT in Section 13.6, $u$ in Section 18.1, and $\eta$ in Section 18.3 below.

[24]: \%\%witefile -a \$Ccodesdir/Hamiltonian-Hreal_on_top.txt

Q4 $=2 * \operatorname{pr} T * \operatorname{pr} T * \operatorname{pr} T * \operatorname{pr} T * u * u *(4-3 * e t a) * e t a$

Appending to SEOBNR/Hamiltonian-Hreal_on_top.txt

\section{Step 7: The $H_{\mathrm{D}}$ Terms [Back to Section 0]}

Recall we defined $H_{\mathrm{D}}$ in Section 4.3 as

$$
H_{\mathrm{D}}=H_{\mathrm{D}} \text { coeffecient } * H_{\mathrm{D}} \text { sum. }
$$

In this step we break down each of $H_{\mathrm{D}}$ coefficient (defined in Section 8.1) and $H_{\mathrm{D}}$ sum (defined in Section 8.2).

\subsection{Step 7.a: $H_{\mathrm{D}}$ Coefficient [Back to Section 0 ]}

From our definition of $H_{\mathrm{D}}$ in Section 4.3, we have

$$
H_{\mathrm{D}} \text { coefficient }=\frac{\mu}{2 M r^{3}},
$$

and recalling the definition of Section 18.3 we'll write

$$
H_{\mathrm{D}} \text { coefficient }=\frac{\eta}{2 r^{3}}
$$

We define $\eta$ in Section 18.3 and $r$ in Section 18.2.

[25]: \%\%writefile -a \$Ccodesdir/Hamiltonian-Hreal_on_top.txt

Hdcoeff $=$ sp.Rational $(1,2) /(r * r * r)$

Appending to SEOBNR/Hamiltonian-Hreal_on_top.txt

\subsection{Step 7.b: $H_{\mathrm{D}}$ Sum [Back to Section 0$]$}

From our definition of $H_{\mathrm{D}}$ in Section 4.3, we have

$$
\begin{aligned}
H_{\mathrm{D} \text { sum }} & =\left(\delta^{i j}-3 n^{i} n^{j}\right) S_{i}^{*} S_{j}^{*} \\
& =\underbrace{\delta^{i j} S_{i}^{*} S_{j}^{*}}_{\text {Term 1 }}-\underbrace{3 n^{i} n^{j} S_{i}^{*} S_{j}^{*}}_{\text {Term 2 }} .
\end{aligned}
$$

We compute $H_{\mathrm{D}}$ Term 1 in Section 8.2.1 and $H_{\mathrm{D}}$ Term 2 in Section 8.2.2.

[26]: \%\%writefile -a \$Ccodesdir/Hamiltonian-Hreal_on_top.txt 
Hdsum $=$ HdsumTerm1 - HdsumTerm2

Appending to SEOBNR/Hamiltonian-Hreal_on_top.txt

8.2.1 Step 7.b.i: $H_{\mathrm{D}}$ Sum Term 1 [Back to Section 0$]$

From our definition of $H_{\mathrm{D}}$ sum Term 1 in Section 8.2, we have

$H_{\mathrm{D}}$ sum Term $1=\delta^{i j} S_{i}^{*} S_{j}^{*}$

where $\delta^{i j}$ is the Kronecker delta:

$$
\delta_{i j}= \begin{cases}0, & i \neq j \\ 1, & i=j .\end{cases}
$$

Thus we have

$$
H_{\mathrm{D}} \text { sum Term } 1=S_{1}^{*} S_{1}^{*}+S_{2}^{*} S_{2}^{*}+S_{3}^{*} S_{3}^{*}
$$

We define $\mathbf{S}^{*}$ in Section 10

[27]: \%\%rritefile -a \$Ccodesdir/Hamiltonian-Hreal_on_top.txt

HdsumTerm $1=$ Sstar $1 *$ Sstar $1+$ Sstar2 $*$ Sstar $2+$ Sstar $3 *$ Sstar 3

Appending to SEOBNR/Hamiltonian-Hreal_on_top.txt

8.2.2 Step 7.b.ii: $H_{\mathrm{D}}$ Sum Term 2 [Back to Section 0$]$

From our definition of $H_{\mathrm{D}}$ sum Term 2 in Section 8.2, we have

$$
\begin{aligned}
H_{\mathrm{D}} \operatorname{sum} \text { Term } 2 & =3 n^{i} n^{j} S_{i}^{*} S_{j}^{*} \\
& =3\left(\mathbf{S}^{*} \cdot \mathbf{n}\right)^{2}
\end{aligned}
$$

We define $\mathbf{S}^{*} \cdot \mathbf{n}$ in Section 9.6.

[28]: \%\%writefile -a \$Ccodesdir/Hamiltonian-Hreal_on_top.txt

HdsumTerm2 $=3 *$ Sstardotn $*$ Sstardotn

Appending to SEOBNR/Hamiltonian-Hreal_on_top.txt

\section{Step 8: Common Dot Products [Back to Section 0]}

What follows are definitions of many common dot products.

9.1 Step 8.a: $\mathrm{S} \cdot \mathrm{S}_{\text {Kerr }}$ [Back to Section 0]

We have

$$
\mathbf{S} \cdot \mathbf{S}_{\text {Kerr }}=S^{1} S_{\text {Kerr }}^{1}+S^{2} S_{\text {Kerr }}^{2}+S^{3} S_{\text {Kerr }}^{3}
$$

We define $\mathbf{S}_{\text {Kerr }}$ in Section 17.4

[29]: \%\%ritefile -a \$Ccodesdir/Hamiltonian-Hreal_on_top.txt

SdotSkerr $=$ S1 $*$ Skerr $1+$ S2 $*$ Skerr2 + S3 $*$ Skerr 3

Appending to SEOBNR/Hamiltonian-Hreal_on_top.txt

9.2 Step 8.b: $\mathrm{S} \cdot \xi$ [Back to Section 0$]$

We have

$\mathbf{S} \cdot \xi=S^{1} \tilde{\xi}^{1}+S^{2} \tilde{\xi}^{2}+S^{3} \xi^{3}$

We define $\xi$ in Section 16.2 .

[30]: \%\%writefile -a \$Ccodesdir/Hamiltonian-Hreal_on_top.txt

Sdotxi $=\mathrm{S} 1 * \mathrm{xi1}+\mathrm{S} 2 * \mathrm{xi} 2+\mathrm{S} 3 * \mathrm{xi} 3$ 
Appending to SEOBNR/Hamiltonian-Hreal_on_top.txt

9.3 Step 8.c: S · v [Back to Section 0]

We have

$$
\mathbf{S} \cdot \mathbf{v}=S^{1} v^{1}+S^{2} v^{2}+S^{3} v^{3}
$$

We define $\mathbf{v}$ in Section 16.1.

[31]: \% \%ritefile -a \$Ccodesdir/Hamiltonian-Hreal_on_top.txt

$\mathrm{Sdotv}=\mathrm{S} 1 * \mathrm{v} 1+\mathrm{S} 2 * \mathrm{v} 2+\mathrm{S} 3 * \mathrm{v} 3$

Appending to SEOBNR/Hamiltonian-Hreal_on_top.txt

9.4 Step 8.d: S · n [Back to Section 0]

We have

$$
\mathbf{S} \cdot \mathbf{n}=S^{1} n^{1}+S^{2} n^{2}+S^{3} n^{3} .
$$

We define $\mathbf{n}$ in Section 16.4 .

[32]: \%\%writefile -a \$Ccodesdir/Hamiltonian-Hreal_on_top.txt

$\mathrm{Sdotn}=\mathrm{S} 1 * \mathrm{n} 1+\mathrm{S} 2 * \mathrm{n} 2+\mathrm{S} 3 * \mathrm{n} 3$

Appending to SEOBNR/Hamiltonian-Hreal_on_top.txt

9.5 Step 8.e: $\mathrm{S} \cdot \hat{\mathrm{S}}_{\text {Kerr }}$ [Back to Section 0]

We have

$$
\mathbf{S} \cdot \hat{\mathbf{S}}_{\text {Kerr }}=S^{1} \hat{S}_{\text {Kerr }}^{1}+S^{2} \hat{S}_{\text {Kerr }}^{2}+S^{3} \hat{S}_{\text {Kerr }}^{3}
$$

We define $\hat{\mathbf{S}}_{\text {Kerr }}$ in Section 17.2.

[33]: \%\%writefile -a \$Ccodesdir/Hamiltonian-Hreal_on_top.txt

SdotSkerrhat $=$ S1*Skerrhat1 + S2*Skerrhat2 + S3*Skerrhat3

Appending to SEOBNR/Hamiltonian-Hreal_on_top.txt

9.6 Step 8.f: $\mathrm{S}^{*} \cdot \mathbf{n}$ [Back to Section 0]

We have

$$
\mathbf{S}^{*} \cdot \mathbf{n}=\mathbf{S}_{1}^{*} n_{1}+\mathbf{S}_{2}^{*} n_{2}+\mathbf{S}_{3}^{*} n_{3} .
$$

We define $\mathbf{S}^{*}$ in Section 10.1 and $\mathbf{n}$ in Section 16.4

[34]: \%\%ritefile -a \$Ccodesdir/Hamiltonian-Hreal_on_top.txt

Sstardotn $=$ Sstar $1 * \mathrm{n} 1+$ Sstar $2 * \mathrm{n} 2+$ Sstar $3 * \mathrm{n} 3$

Appending to SEOBNR/Hamiltonian-Hreal_on_top.txt

10 Step 9: $H_{\text {real }}$ Spin Combination $\mathbf{S}^{*}$ [Back to Section 0]

We collect here terms defining and containing $\mathbf{S}^{*}$.

10.1 Step 9.a: S* [Back to Section 0]

From BB2010 Equation (5.63):

$$
\mathbf{S}^{*}=\sigma^{*}+\frac{1}{c^{2}} \Delta_{\sigma^{*}}
$$

We define $\sigma^{*}$ in Section 17.6 and $\boldsymbol{\Delta}_{\sigma^{*}}$ in Section 10.2

Please note: after normalization, $\mathbf{S}=\mathbf{S}^{*}$. See BB2010 Equation (4.26) 
[35]: \%\%writefile -a \$Ccodesdir/Hamiltonian-Hreal_on_top.txt

S1 = Sstar 1
S2 = Sstar2
S3 = Sstar3
Sstar1 = sigmastar1 + Deltasigmastar1
Sstar2 = sigmastar2 + Deltasigmastar2

Sstar3 $=$ sigmastar $3+$ Deltasigmastar3

Appending to SEOBNR/Hamiltonian-Hreal_on_top.txt

10.2 Step 9.b: $\Delta_{\sigma^{*}}[$ Back to Section 0]

We can write $\boldsymbol{\Delta}_{\sigma^{*}}$ as

$$
\Delta_{\sigma^{*}}=\sigma^{*}\left(\sigma^{*} \text { coefficient }\right)+\sigma(\sigma \text { coefficient })
$$

For further dissection, see $\sigma^{*}$ in Section 17.6, $\sigma^{*}$ coefficient in Section 10.3, $\sigma$ in Section 17.5, and $\sigma$ coefficient in Section 10.4.

[36]: \%\%writefile -a \$Ccodesdir/Hamiltonian-Hreal_on_top.txt

Deltasigmastar1 $=$ sigmastar $1 *$ sigmastarcoeff + sigma1 $*$ sigmacoeff
Deltasigmastar2 $=$ sigmastar $2 *$ sigmastarcoeff + sigma2*sigmacoeff

Deltasigmastar2 $=$ sigmastar2*sigmastarcoeff + sigma2*sigmacoeff
Deltasigmastar3 $=$ sigmastar $3 *$ sigmastarcoeff + sigma3*sigmacoeff

Appending to SEOBNR/Hamiltonian-Hreal_on_top.txt

10.3 Step 9.c: $\sigma^{*}$ coefficient [Back to Section 0]

We will break down $\sigma^{*}$ coefficient into two terms:

$\sigma^{*}$ coefficient $=\sigma^{*}$ coefficient Term $1+\sigma^{*}$ coefficient Term 2

We compute $\sigma^{*}$ coefficient Term 1 in Section 10.3 .1 and $\sigma^{*}$ coefficient Term 2 in Section 10.3.2.

[37]: \%\%rritefile -a \$Ccodesdir/Hamiltonian-Hreal_on_top.txt

sigmastarcoeff $=$ sigmastarcoeffTerm1 + sigmastarcoeffTerm2

Appending to SEOBNR/Hamiltonian-Hreal_on_top.txt

10.3.1 Step 9.c.i: $\sigma^{*}$ Coefficient Term 1 [Back to Section 0]

We build this term from BB2011 Equation (51) with $b_{0}=0$ (see discussion preceeding T2012 Equation (4)), where what is listed below is the coefficient on $\sigma^{*}$ :

$$
\begin{aligned}
\sigma^{*} \text { coefficient Term } 1 & =\frac{7}{6} \eta \frac{M}{r}+\frac{1}{3} \eta(Q-1)-\frac{5}{2} \eta \frac{\Delta_{r}}{\Sigma}(\mathbf{n} \cdot \hat{\mathbf{p}})^{2} \\
& =\frac{\eta}{12}\left(14 \frac{M}{r}+4(Q-1)-30 \frac{\Delta_{r}}{\Sigma}(\mathbf{n} \cdot \hat{\mathbf{p}})^{2}\right)
\end{aligned}
$$

We group together and compute $Q-1$ in Section 12.7 and $\frac{\Delta_{r}}{\Sigma}(\mathbf{n} \cdot \hat{\mathbf{p}})^{2}$ in Section 12.7.1; we define $r$ in Section 18.2, $\eta$ in Section 18.3, and $M$ in Section 18.5 below. [38]: \%\%writefile -a \$Ccodesdir/Hamiltonian-Hreal_on_top.txt

sigmastarcoeffTerm1 $=$ eta $/ 12 *(14 / r+4 * Q \operatorname{minus} 1-30 * \operatorname{DrSipn} 2)$

Appending to SEOBNR/Hamiltonian-Hreal_on_top.txt

10.3.2 Step 9.c.ii: $\sigma^{*}$ Coefficient Term 2 [Back to Section 0]

We build this term from BB2011 Equation (52) with all $b_{i}=0, i \in\{0,1,2,3\}$ (see discussion preceeding T2012 Equation (4)), and just the coefficient on $\sigma^{*}$. In the LALSuite code this is the variable 'sMultiplier1'. 


$$
\begin{aligned}
\sigma^{*} \text { coefficient Term } 2= & \frac{1}{36}\left(353 \eta-27 \eta^{2}\right)\left(\frac{M}{r}\right)^{2}+\frac{5}{3}\left(3 \eta^{2}\right) \frac{\Delta_{r}^{2}}{\Sigma^{2}}(\mathbf{n} \cdot \hat{\mathbf{p}})^{4} \\
& +\frac{1}{72}\left(-23 \eta-3 \eta^{2}\right)(Q-1)^{2}+\frac{1}{36}\left(-103 \eta+60 \eta^{2}\right) \frac{M}{r}(Q-1) \\
& +\frac{1}{12}\left(16 \eta-21 \eta^{2}\right) \frac{\Delta_{r}}{\Sigma}(\mathbf{n} \cdot \hat{\mathbf{p}})^{2}(Q-1)+\frac{1}{12}\left(47 \eta-54 \eta^{2}\right) \frac{M}{r} \frac{\Delta_{r}}{\Sigma}(\mathbf{n} \cdot \hat{\mathbf{p}})^{2} \\
= & \frac{\eta}{72 r^{2}}\left[(706-54 \eta) M^{2}+360 \eta r^{2} \frac{\Delta_{r}^{2}}{\Sigma^{2}}(\mathbf{n} \cdot \hat{\mathbf{p}})^{4}+r^{2}(-23-3 \eta)(Q-1)^{2}+(-206+120 \eta) M r(Q-1)\right. \\
& \left.+(96-126 \eta) r^{2} \frac{\Delta_{r}}{\Sigma}(\mathbf{n} \cdot \hat{\mathbf{p}})^{2}(Q-1)+(282-324 \eta) M r \frac{\Delta_{r}}{\Sigma}(\mathbf{n} \cdot \hat{\mathbf{p}})^{2}\right] \\
= & \frac{\eta}{72 r^{2}}\left[706+r\left(-206 M(Q-1)+282 M \frac{\Delta_{r}}{\Sigma}(\mathbf{n} \cdot \hat{\mathbf{p}})^{2}+r(Q-1)\left(96 \frac{\Delta_{r}}{\Sigma}(\mathbf{n} \cdot \hat{\mathbf{p}})^{2}-23(Q-1)\right)\right)\right. \\
& +\eta\left(-54 M^{2}+r\left(120 M(Q-1)-324 M \frac{\Delta_{r}}{\Sigma}(\mathbf{n} \cdot \hat{\mathbf{p}})^{2}\right.\right. \\
& \left.\left.\left.+r\left(360 \frac{\Delta_{r}^{2}}{\Sigma^{2}}(\mathbf{n} \cdot \hat{\mathbf{p}})^{4}+(Q-1)\left(-126 \frac{\Delta_{r}}{\Sigma}(\mathbf{n} \cdot \hat{\mathbf{p}})^{2}-3(Q-1)\right)\right)\right)\right)\right]
\end{aligned}
$$

We define $r$ in Section 18.2, $\eta$ in Section 18.3, and $M$ in Section 18.5; we group together and define $Q-1$ in Section 12.7 , and $\frac{\Delta r}{\Sigma}(\mathbf{n} \cdot \hat{\mathbf{p}})^{2}$ in Section 12.7 .1 .

[39]: \%\%ritefile -a \$Ccodesdir/Hamiltonian-Hreal_on_top.txt

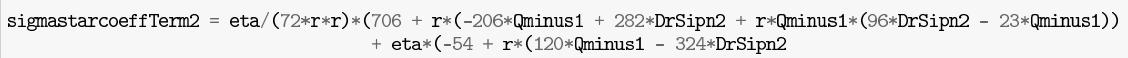

Appending to SEOBNR/Hamiltonian-Hreal_on_top.txt

\subsection{Step 9.d: $\sigma$ coefficient [Back to Section 0]}

We will break down $\sigma$ coefficient into three terms:

$\sigma$ coefficient $=\sigma$ coefficient Term $1+\sigma$ coefficient Term $2+\sigma$ coefficient Term 3

We compute $\sigma$ coefficient Term 1 in Section 10.4.1, $\sigma$ coefficient Term 2 in Section 10.4.2, and $\sigma$ coefficient Term 3 in Section 10.4.3.

[40]: \%\%ritefile -a \$Ccodesdir/Hamiltonian-Hreal_on_top.txt

sigmacoeff $=$ sigmacoeffTerm $1+$ sigmacoeffTerm2 + sigmacoeffTerm3

$$
27
$$

Appending to SEOBNR/Hamiltonian-Hreal_on_top.txt

10.4.1 Step 9.d.i: $\sigma$ Coefficient Term 1 [Back to Section 0$]$

We build this term from BB2011 Equation (51) with $a_{0}=0$ (see discussion preceeding T2012 Equation (4)), where what is listed below is the coefficient on $\sigma$ :

$$
\begin{aligned}
\sigma \text { coefficient Term } 1 & =-\frac{2}{3} \eta \frac{M}{r}+\frac{1}{4} \eta(Q-1)-3 \eta \frac{\Delta_{r}}{\Sigma}(\mathbf{n} \cdot \hat{\mathbf{p}})^{2} \\
& =\frac{\eta}{12}(-8 \frac{M}{r}+3(Q-1)-36 \underbrace{\frac{\Delta_{r}}{\Sigma}(\mathbf{n} \cdot \hat{\mathbf{p}})^{2}}_{\text {DrSipn2 }})
\end{aligned}
$$

We define $\eta$ in Section 18.3, $M$ in Section 18.5, $Q-1$ in Section 12.7, and $\frac{\Delta r}{\Sigma}(\mathbf{n} \cdot \hat{\mathbf{p}})^{2}$ in Section 12.7.1.

[41]: \%\%ritefile -a \$Ccodesdir/Hamiltonian-Hreal_on_top.txt

sigmacoeffTerm1 $=$ eta $/ 12 *(-8 / \mathrm{r}+3 *$ Qminus $1-36 * \operatorname{DrSipn} 2)$

Appending to SEOBNR/Hamiltonian-Hreal_on_top.txt

10.4.2 Step 9.d.ii: $\sigma$ Coefficient Term 2 [Back to Section 0]

We build this term from BB2011 Equation (52) with all $a_{i}=0, i \in\{0,1,2,3\}$ (see discussion preceeding T2012 Equation (4)), and just the coefficient on $\sigma$ :

$$
\begin{aligned}
\sigma \text { coefficient Term 2 }= & \frac{1}{9}\left(-56 \eta-21 \eta^{2}\right)\left(\frac{M}{r}\right)^{2}+\frac{5}{24}\left(27 \eta^{2}\right) \frac{\Delta_{r}^{2}}{\Sigma^{2}}(\mathbf{n} \cdot \hat{\mathbf{p}})^{4} \\
& +\frac{1}{144}(-45 \eta)(Q-1)^{2}+\frac{1}{36}\left(-109 \eta+51 \eta^{2}\right) \frac{M}{r}(Q-1) \\
& +\frac{1}{24}\left(6 \eta-39 \eta^{2}\right) \frac{\Delta_{r}}{\Sigma}(\mathbf{n} \cdot \hat{\mathbf{p}})^{2}(Q-1)+\frac{1}{24}\left(-16 \eta-147 \eta^{2}\right) \frac{M}{r} \frac{\Delta_{r}}{\Sigma}(\mathbf{n} \cdot \hat{\mathbf{p}})^{2} \\
= & \frac{\eta}{144 r^{2}}\left[-896 M^{2}+r\left(-436 M(Q-1)-96 M \frac{\Delta_{r}}{\Sigma}(\mathbf{n} \cdot \hat{\mathbf{p}})^{2}\right.\right. \\
& \left.+r\left(-45(Q-1)^{2}+36(Q-1) \frac{\Delta_{r}}{\Sigma}(\mathbf{n} \cdot \hat{\mathbf{p}})^{2}\right)\right)+\eta\left(-336 M^{2}+r\left(204 M(Q-1)-882 M \frac{\Delta_{r}}{\Sigma}(\mathbf{n} \cdot \hat{\mathbf{p}})^{2}\right.\right. \\
& \left.\left.\left.+r\left(810 \frac{\Delta_{r}^{2}}{\Sigma^{2}}(\mathbf{n} \cdot \hat{\mathbf{p}})^{4}-234(Q-1) \frac{\Delta_{r}}{\Sigma}(\mathbf{n} \cdot \hat{\mathbf{p}})^{2}\right)\right)\right)\right]
\end{aligned}
$$


We define $\eta$ in Section 18.3, $M$ in Section 18.5, $Q-1$ in Section 12.7, and $\frac{\Delta_{r}}{\Sigma}(\mathbf{n} \cdot \hat{\mathbf{p}})^{2}$ in Section 12.7.1.

[42]: \%\%ritefile -a \$Ccodesdir/Hamiltonian-Hreal_on_top.txt

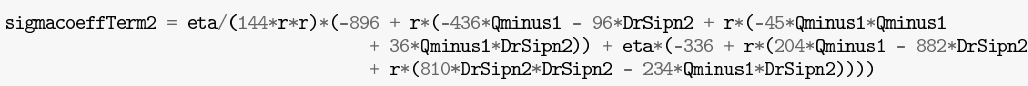

Appending to SEOBNR/Hamiltonian-Hreal_on_top.txt

10.4.3 Step 9.d.iii: $\sigma$ Coefficient Term 3 [Back to Section 0]

From Section II of T2014)

$\sigma$ coefficient Term $3=\eta d S O u^{3}$.

where $d_{\text {SO }}$ is a fitting parameter. We define $\eta$ in Section 18.3, $u$ in Section 18.1.

[43]: \%\%writefile -a \$Ccodesdir/Hamiltonian-Hreal_on_top.txt

sigmacoeffTerm $3=$ eta $* d S 0 * u * u * u$

$\mathrm{dSO}=-74.71-156 . *$ eta $+627.5 *$ eta $*$ eta

Appending to SEOBNR/Hamiltonian-Hreal_on_top.txt

\section{Step 10: Derivatives of the Metric Potential [Back to Section 0]}

We collect here terms dependent on derivatives of the metric potential (see BB2010 Equations (5.47))

11.1 Step 10.a: $\omega_{r}$ [Back to Section 0]

From BB2010 Equation (5.47b) we have

$$
\omega_{r}=\frac{\Lambda_{t} \tilde{\omega}_{\mathrm{fd}}^{\prime}-\Lambda_{t}^{\prime} \tilde{\omega}_{\mathrm{fd}}}{\Lambda_{t}^{2}}
$$

29

We define $\Lambda_{t}$ in Section 14.1, $\tilde{\omega}_{\mathrm{fd}}^{\prime}$ in Section 11.8, $\Lambda_{t}^{\prime}$ in Section 11.7, and $\tilde{\omega}_{\mathrm{fd}}$ in Section 14.6.

[44]: \%\%writefile -a \$Ccodesdir/Hamiltonian-Hreal_on_top.txt

omegar $=($ Lambdat $*$ omegatildeprm - Lambdatprm*omegatilde $) /($ Lambdat $*$ Lambdat $)$

Appending to SEOBNR/Hamiltonian-Hreal_on_top.txt

11.2 Step 10.b: $v_{r}$ [Back to Section 0$]$

From BB2010 Equation (5.47c) we have

$$
v_{r}=\frac{r}{\Sigma}+\frac{\omega^{2}\left(\omega^{2} \Delta_{t}^{\prime}-4 r \Delta_{t}\right)}{2 \Lambda_{t} \Delta_{t}} .
$$

We define $r$ in Section 18.2, $\Sigma$ in Section 15.1, $\omega^{2}$ in Section 15.2, $\Delta_{t}^{\prime}$ in Section 14.4, $\Delta_{t}$ in Section 14.3, and $\Lambda_{t}$ in Section 14.1.

[45]: \%\%rritefile -a \$Ccodesdir/Hamiltonian-Hreal_on_top.txt

nur $=\mathrm{r} /$ Sigma $+\mathrm{w} 2 *(\mathrm{w} 2 *$ Deltatprm $-4 * \mathrm{r} *$ Deltat $) /(2 *$ Lambdat $*$ Deltat $)$

Appending to SEOBNR/Hamiltonian-Hreal_on_top.txt

11.3 Step 10.c: $\mu_{r}$ [Back to Section 0]

From BB2010 Equation (5.47d) we have

$$
\mu_{r}=\frac{r}{\Sigma}-\frac{1}{\sqrt{\Delta_{r}}} .
$$

We define $r$ in Section 18.2, $\Sigma$ in Section 15.1, and $\Delta_{r}$ in Section 14.2.

[46]: \%/writefile -a \$Ccodesdir/Hamiltonian-Hreal_on_top.txt

mur $=r /$ Sigma $-1 /$ sp $\cdot$ sqrt $($ Deltar $)$

Appending to SEOBNR/Hamiltonian-Hreal_on_top.txt 
11.4 Step 10.d: $\omega_{\cos \theta}$ [Back to Section 0]

From BB2010 Equation (5.47f), we have

$$
\omega_{\cos \theta}=-\frac{2 a^{2} \cos \theta \Delta_{t} \tilde{\omega}_{\mathrm{fd}}}{\Lambda_{t}^{2}} .
$$

We define $a$ in Section 17.1, $\cos \theta$ in Section 15.4, $\Delta_{t}$ in Section 14.3, $\tilde{\omega}_{\mathrm{fd}}$ in Section 14.6, and $\Lambda_{t}$ in Section 14.1.

[47]: \%\%writefile -a \$Ccodesdir/Hamiltonian-Hreal_on_top.txt

omegacostheta $=-2 * a * a * \cos$ theta $*$ Deltat $*$ omegatilde $/($ Lambdat $*$ Lambdat $)$

Appending to SEOBNR/Hamiltonian-Hreal_on_top.txt

11.5 Step 10.e: $v_{\cos \theta}$ [Back to Section 0]

From BB2010 Equation (5.47g) we have

$$
v_{\cos \theta}=\frac{a^{2} \omega^{2} \cos \theta\left(\omega^{2}-\Delta_{t}\right)}{\Lambda_{t} \Sigma} .
$$

We define $a$ in Section 17.1, $\omega^{2}$ in Section 15.2, $\cos \theta$ in Section 15.4, $\Delta_{t}$ in Section 14.3, $\Lambda_{t}$ in Section 14.1, and $\Sigma$ in Section 15.1.

[48]: \%\%ritefile -a \$Ccodesdir/Hamiltonian-Hreal_on_top.txt

nucostheta $=\mathrm{a} * \mathrm{a} * \mathrm{w} 2 * \cos$ theta $*(\mathrm{w} 2-$ Deltat $) /($ Lambdat $*$ Sigma $)$

Appending to SEOBNR/Hamiltonian-Hreal_on_top.txt

11.6 Step 10.f: $\mu_{\cos \theta}$ [Back to Section 0$]$

From BB2010 Equation (5.47h) we have

$$
\mu_{\cos \theta}=\frac{a^{2} \cos \theta}{\Sigma} .
$$

31

We define $a$ in Section 17.1, $\cos \theta$ in Section 15.4, and $\Sigma$ in Section 15.1 below.

[49]: \%\%ritefile -a \$Ccodesdir/Hamiltonian-Hreal_on_top.txt

mucostheta $=\mathrm{a} * \mathrm{a} * \cos$ theta $/$ Sigma

Appending to SEOBNR/Hamiltonian-Hreal_on_top.txt

\subsection{Step 10.g: $\Lambda_{t}^{\prime}$ [Back to Section 0]}

From the discussion after BB2010 Equations (5.47), we know that the prime notation indicates a derivative with respect to $r$. Using the definiton of $\Lambda_{t}$ in Section 14.1 we have

$$
\Lambda_{t}^{\prime}=4\left(a^{2}+r^{2}\right) r-a^{2} \Delta_{t}^{\prime} \sin ^{2} \theta
$$

We define $a$ in Section 17.1, $r$ in Section 18.2, $\Delta_{u}$ in Section 14.5, and $\sin ^{2} \theta$ in Section 15.3.

[50]: \%\%writefile -a \$Ccodesdir/Hamiltonian-Hreal_on_top.txt

Lambdatprm $=4 *(\mathrm{a} * \mathrm{a}+\mathrm{r} * \mathrm{r}) * \mathrm{r}-2 * \mathrm{a} * \mathrm{a} *$ Deltatprm $* \sin 2$ theta

Appending to SEOBNR/Hamiltonian-Hreal_on_top.txt

11.8 Step 10.h: $\tilde{\omega}_{\text {fd }}^{\prime}$ [Back to Section 0]

From the discussion after BB2010 Equation (5.47), we know that the prime notation indicates a derivative with respect to $r$. Using the definiton of $\tilde{\omega}_{\mathrm{fd}}$ in Section 14.6 , we have

$$
\tilde{\omega}_{\mathrm{fd}}^{\prime}=2 a M
$$

We define $a$ in Section 17.1 and $M$ in Section 18.5

[51]: \%\%ritefile -a \$Ccodesdir/Hamiltonian-Hreal_on_top.txt

omegatildeprm $=2 * a$

Appending to SEOBNR/Hamiltonian-Hreal_on_top.txt 


\section{Step 11: The Deformed and Rescaled Metric Potentials [Back to Section 0]}

We collect here terms of the deformed and scaled metric potentils. See BB2010 Equations (5.30)--(5.34) and (5.48)--(5.52).

\subsection{Step 11.a: $\omega$ [Back to Section 0]}

From BB2010 Equation (5.31) we have

$$
\omega=\frac{\tilde{\omega}_{\mathrm{fd}}}{\Lambda_{t}} .
$$

We define $\tilde{\omega}_{\mathrm{fd}}$ in Section 14.6 and $\Lambda_{t}$ in Section 14.1

[52]: \%\%ritefile -a \$Ccodesdir/Hamiltonian-Hreal_on_top.txt

omega $=$ omegatilde/Lambdat

Appending to SEOBNR/Hamiltonian-Hreal_on_top.txt

12.2 Step 11.b: $e^{2 v}$ and $e^{v}$ [Back to Section 0]

From BB2010 Equation (5.32), we have

$$
e^{2 v}=\frac{\Delta_{t} \Sigma}{\Lambda_{t}}
$$

It follows that

$$
e^{v}=\sqrt{\frac{\Delta_{t} \Sigma}{\Lambda_{t}}}
$$

We define $\Delta_{t}$ in Section 14.3, $\Sigma$ in Section 15.1, and $\Lambda_{t}$ in Section 14.1.

[53]: \%\%ritefile -a \$Ccodesdir/Hamiltonian-Hreal_on_top.txt

$\operatorname{expnu}=\operatorname{sp} \cdot \operatorname{sqrt}(\exp 2$ nu $)$

$\exp 2 \mathrm{nu}=$ Deltat $*$ Sigma $/$ Lambdat

Appending to SEOBNR/Hamiltonian-Hreal_on_top.txt

12.3 Step 11.c: $\tilde{B}$ [Back to Section 0]

From BB2010 Equation (5.48), we have

$$
\tilde{B}=\sqrt{\Delta_{t}} .
$$

We define $\Delta_{t}$ in Section 14.3

[54]: \%/writefile -a \$Ccodesdir/Hamiltonian-Hreal_on_top.txt

Btilde $=$ sp.sqrt $($ Deltat $)$

Appending to SEOBNR/Hamiltonian-Hreal_on_top.txt

12.4 Step 11.d: $\tilde{B}_{r}$ [Back to Section 0]

From BB2010 Equation (5.49), we have

$$
\tilde{B}_{r}=\frac{\sqrt{\Delta_{r}} \Delta_{t}^{\prime}-2 \Delta_{t}}{2 \sqrt{\Delta_{r} \Delta_{t}}} .
$$

We define $\Delta_{r}$ in Section 14.2, $\Delta_{t}^{\prime}$ in Section 14.4, and $\Delta_{t}$ in Section 14.3

[55]: \%\%writefile -a \$Ccodesdir/Hamiltonian-Hreal_on_top.txt

Brtilde $=($ sp.sqrt $($ Deltar $) *$ Deltatprm $-2 *$ Deltat $) /(2 *$ sp. sqrt $($ Deltar $*$ Deltat $))$

Appending to SEOBNR/Hamiltonian-Hreal_on_top.txt 
12.5 Step 11.e: $e^{2 \tilde{\mu}}$ and $e^{\tilde{\mu}}$ [Back to Section 0]

From BB2010 Equation (5.50), we have

$$
e^{2 \tilde{\mu}}=\Sigma \text {. }
$$

It follows that

$$
e^{\tilde{\mu}}=\sqrt{\Sigma} \text {. }
$$

We define $\Sigma$ in Section 15.1.

[56]: \%\%writefile -a \$Ccodesdir/Hamiltonian-Hreal_on_top.txt

expmu $=\mathrm{sp} \cdot \operatorname{sqrt}(\exp 2 \mathrm{mu})$

$\exp 2 \mathrm{mu}=$ Sigma

Appending to SEOBNR/Hamiltonian-Hreal_on_top.txt

12.6 Step 11.f: $\tilde{J}$ [Back to Section 0]

From BB2010 Equation (5.51) we have

$$
\tilde{J}=\sqrt{\Delta_{r}} .
$$

We define $\Delta_{r}$ in Section 14.2 below.

[57]: \%\%ritefile -a \$Ccodesdir/Hamiltonian-Hreal_on_top.txt

Jtilde $=$ sp.sqrt (Deltar)

Appending to SEOBNR/Hamiltonian-Hreal_on_top.txt

12.7 Step 11.g: $Q$ [Back to Section 0]

From BB2010 Equation (5.52),

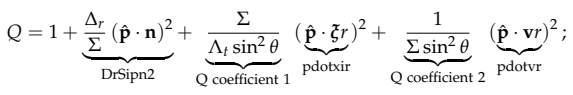

We group togther and compute $\frac{\Delta_{r}}{\Sigma}(\hat{\mathbf{p}} \cdot \mathbf{n})^{2}$ in Section 12.7.1, $\frac{\Sigma}{\Lambda_{t} \sin ^{2} \theta}$ in Section 12.7.2, $\hat{\mathbf{p}} \cdot \boldsymbol{\xi} r$ in Section 13.4, $\frac{1}{\Sigma \sin ^{2} \theta}$ in Section 12.7.3, and $\hat{\mathbf{p}} \cdot \mathbf{v} r$ in Section 13.2.

[58]: \%\%ritefile -a \$Ccodesdir/Hamiltonian-Hreal_on_top.txt

Qminus1 = Q - 1

$Q=1+\operatorname{DrSipn} 2+$ Qcoeff1*pdotxir*pdotxir + Qcoeff2*pdotvr*pdotvr

Appending to SEOBNR/Hamiltonian-Hreal_on_top.txt

12.7.1 Step 11.g.i: $\frac{\Delta_{r}}{\Sigma}(\hat{\mathbf{p}} \cdot \mathbf{n})^{2}[$ Back to Section 0]

We define $\Delta_{r}$ in Section 14.2, $\Sigma$ in Section 15.1, and $\hat{\mathbf{p}} \cdot \mathbf{n}$ in Section 13.3.

[59]: \%\%writefile -a \$Ccodesdir/Hamiltonian-Hreal_on_top.txt

DrSipn2 $=$ Deltar $*$ pdotn $*$ pdotn $/$ Sigma

Appending to SEOBNR/Hamiltonian-Hreal_on_top.txt

12.7.2 Step 11.g.ii: Q Coefficient 1 [Back to Section 0]

We defined $Q$ coefficient 1 in Section 12.7 as

$$
Q \text { coefficient } 1=\frac{\Sigma}{\Lambda_{t} \sin ^{2} \theta}
$$

We define $\Sigma$ in Section 15.1, $\Lambda_{t}$ in Section 14.1, and $\sin ^{2} \theta$ in Section 15.3. 
[60]: \%\%writefile -a \$Ccodesdir/Hamiltonian-Hreal_on_top.txt

Qcoeff1 $=$ Sigma $/($ Lambdat $*$ sin2theta $)$

Appending to SEOBNR/Hamiltonian-Hreal_on_top.txt

12.7.3 Step 11.g.iii: $Q$ Coefficient 2 [Back to Section 0$]$

We defined $Q$ coefficient 2 in Section 12.7 as

$$
Q \text { coefficient } 2=\frac{1}{\Sigma \sin ^{2} \theta}
$$

We define $\Sigma$ in Section 15.1 and $\sin ^{2} \theta$ in Section 15.3.

[61]: \%\%ritefile -a \$Ccodesdir/Hamiltonian-Hreal_on_top.txt

Qcoeff2 $=1 /($ Sigma $*$ sin2theta $)$

Appending to SEOBNR/Hamiltonian-Hreal_on_top.txt

\section{Step 12: Tortoise Terms [Back to Section 0]}

We collect here terms related to the conversion from Boyer-Lindquist coordinates to tortoise coordinates. Details of the converstion are given in the appendix of P2010

13.1 Step 12.a: $p_{\phi}$ [Back to Section 0]

From the discussion preceding BB2010 Equation (3.41), the phi component of the tortoise momentum $p_{\phi}$ is given by

$$
p_{\phi}=\hat{\mathbf{p}} \cdot \boldsymbol{\xi} r
$$

We define $\hat{\mathbf{p}} \cdot \xi r$ in Section 13.4

[62]: \%\%writefile -a \$Ccodesdir/Hamiltonian-Hreal_on_top.txt

pphi $=$ pdotxir

Appending to SEOBNR/Hamiltonian-Hreal_on_top.txt

13.2 Step 12.b: $\hat{\mathbf{p}} \cdot \mathbf{v} r$ [Back to Section 0]

We have

$$
\hat{\mathbf{p}} \cdot \mathbf{v} r=\left(\hat{p}_{1} v_{1}+\hat{p}_{2} v_{2}+\hat{p}_{3} v_{3}\right) r
$$

We define $\hat{\mathbf{p}}$ in Section 13.5, $\mathbf{v}$ in Section 16.1, and $r$ in Section 18.2.

[63]: \%\%ritefile -a \$Ccodesdir/Hamiltonian-Hreal_on_top.txt

pdotvr $=($ phat $1 * \mathrm{v} 1+$ phat $2 * \mathrm{v} 2+$ phat $3 * \mathrm{v} 3) * \mathrm{r}$

Appending to SEOBNR/Hamiltonian-Hreal_on_top.txt

13.3 Step 12.c: $\hat{\mathbf{p}} \cdot \mathbf{n}[$ [Back to Section 0]

We have

$$
\hat{\mathbf{p}} \cdot \mathbf{n}=\hat{p}_{1} n_{1}+\hat{p}_{2} n_{2}+\hat{p}_{3} n_{3}
$$

We define $\hat{\mathbf{p}}$ in Section 13.5 and $\mathbf{n}$ in Section 16.4

[64]: \%\%writefile -a \$Ccodesdir/Hamiltonian-Hreal_on_top.txt

pdotn $=$ phat $1 * \mathrm{n} 1+$ phat $2 * \mathrm{n} 2+$ phat $3 * \mathrm{n} 3$

Appending to SEOBNR/Hamiltonian-Hreal_on_top.txt 
We have

We define $\hat{\mathbf{p}}$ in Section 13.5, $\boldsymbol{\xi}$ in Section 16.2, and $r$ in Section 18.2.

[65]: \%writefile -a \$Ccodesdir/Hamiltonian-Hreal_on_top.txt

pdotxir $=($ phat $1 * x i 1+$ phat $2 * x i 2+$ phat $3 * x i 3) * r$

Appending to SEOBNR/Hamiltonian-Hreal_on_top.txt

13.5 Step 12.e: $\hat{p}$ [Back to Section 0]

From the discussion after BB2010 Equation (3.41), we have $\hat{\mathbf{p}}=\mathbf{p} / m$ where $m$ is the mass of a nonspinning test particle and $\mathbf{p}$ is conjugate momentum. Following Lines 319--321 of LALSimIMRSpinEOBHamiltonianPrec.c, we convert the Boyer-Lindquist momentum $\mathbf{p}$ to the tortoise momentum (see the appendix of P2010) via

$$
\hat{\mathbf{p}}=\mathbf{p}+\operatorname{prT}\left(1-\frac{1}{\operatorname{csi1}}\right) \mathbf{n}
$$

We define prT in Section 13.6, csi1 in Section 13.8, and $\mathbf{n}$ in Section 16.4.

[66]: \%\%ritefile -a \$Ccodesdir/Hamiltonian-Hreal_on_top.txt

phat $1=\mathrm{p} 1+\operatorname{prT} *(1-1 / \operatorname{csi} 1) * \mathrm{n} 1$
phat $2=\mathrm{p} 2+\operatorname{prT} *(1-1 / \operatorname{csi} 1) * \mathrm{n} 2$

phat $2=$ p $2+\operatorname{prT} *(1-1 / \operatorname{csi} 1) *$ n 2

phat $3=p 3+\operatorname{prT} *(1-1 / \operatorname{csi} 1) * n 3$

Appending to SEOBNR/Hamiltonian-Hreal_on_top.txt

13.6 Step 12.f: prT [Back to Section 0]

The first component of the momenum vector, after conversion to tortoise coordinates (see the Appendix of P2010), is

We define csi2 in Section 13.7 and $\mathbf{n}$ in Section 16.4

[67]: \%\%writefile -a \$Ccodesdir/Hamiltonian-Hreal_on_top.txt

$\operatorname{pr} \mathrm{T}=\operatorname{csi} 2 *(\mathrm{p} 1 * \mathrm{n} 1+\mathrm{p} 2 * \mathrm{n} 2+\mathrm{p} 3 * \mathrm{n} 3)$

Appending to SEOBNR/Hamiltonian-Hreal_on_top.txt

13.7 Step 12.g: csi2 [Back to Section 0]

From the transformation to tortoise coordinates in the Appendix of P2010,

$$
\operatorname{csi} 2=1+\left(\frac{1}{2}-\frac{1}{2} \operatorname{sign}\left(\frac{3}{2}-\tau\right)\right)(\operatorname{csi}-1)
$$

We define csi in Section 13.9; $\tau$ is a tortoise coordinate $(\tau \in\{0,1,2\})$.

[68]: \%\%writefile -a \$Ccodesdir/Hamiltonian-Hreal_on_top.txt

$\operatorname{csi} 2=1+($ sp. Rational $(1,2)-$ sp.Rational $(1,2) * s p \cdot \operatorname{sign}($ sp.Rational $(3,2)-$ tortoise $)) *(\operatorname{csi}-1)$

Appending to SEOBNR/Hamiltonian-Hreal_on_top.txt

13.8 Step 12.h: csi1 [Back to Section 0]

From the transformation to tortoise coordinates in the Appendix of P2010,

$$
\operatorname{csi} 1=1+(1-|1-\tau|)(\operatorname{csi}-1)
$$

We define csi in Section 13.9; $\tau$ is a tortoise coordinate $(\tau \in\{0,1,2\})$.

[69]: \%\%ritefile -a \$Ccodesdir/Hamiltonian-Hreal_on_top.txt

$\operatorname{csi1}=1+(1-\operatorname{sp} \cdot \operatorname{abs}(1-$ tortoise $)) *(\operatorname{csi}-1)$

Appending to SEOBNR/Hamiltonian-Hreal_on_top.txt 
From the transformation to tortoise coordinates in the Appendix of P2010,

$$
\operatorname{csi}=\frac{\sqrt{\Delta_{t} \Delta_{r}}}{\omega^{2}} .
$$

We define $\Delta_{t}$ in Section 14.3, $\Delta_{r}$ in Section 14.2, and $\omega^{2}$ in Section 15.2.

[70]: \%\%writefile -a \$Ccodesdir/Hamiltonian-Hreal_on_top.txt

$\operatorname{csi}=\operatorname{sp} \cdot$ sqrt $($ Deltar $*$ Deltat $) /$ w2

Appending to SEOBNR/Hamiltonian-Hreal_on_top.txt

\section{Step 13: Metric Terms [Back to Section 0]}

We collect here terms used to define the deformed Kerr metric. See BB2010 Equations (5.38)--(5.40) and (5.71)--(5.75).

14.1 Step 13.a: $\Lambda_{t}$ [Back to Section 0]

From BB2010 Equation (5.39),

$$
\Lambda_{t}=\omega^{4}-a^{2} \Delta_{t} \sin ^{2} \theta .
$$

We define $\mathscr{\omega}^{2}$ in Section 15.2, $a$ in Section 17.1, $\Delta_{t}$ in Section 14.3, and $\sin ^{2} \theta$ in Section 15.3.

[71]: \%\%ritefile -a \$Ccodesdir/Hamiltonian-Hreal_on_top.txt

Lambdat $=$ w2 $*$ w2 $-\mathrm{a} * \mathrm{a} *$ Deltat $*$ sin2theta

Appending to SEOBNR/Hamiltonian-Hreal_on_top.txt

14.2 Step 13.b: $\Delta_{r}$ [Back to Section 0]

From BB2010 Equation (5.38),

$$
\Delta_{r}=\Delta_{t} D^{-1}
$$

We define $\Delta_{t}$ in Section 14.3 and $D^{-1}$ in Section 14.7.

[72]: \%\%writefile -a \$Ccodesdir/Hamiltonian-Hreal_on_top.txt

Deltar $=$ Deltat $*$ Dinv

Appending to SEOBNR/Hamiltonian-Hreal_on_top.txt

14.3 Step 13.c: $\Delta_{t}$ [Back to Section 0$]$

From BB2010 Equation (5.71), we have

$$
\Delta_{t}=r^{2} \Delta_{u}
$$

We define $\Delta_{u}$ in Section 14.5 and $r$ in Section 18.2

[73]: \%/writefile -a \$Ccodesdir/Hamiltonian-Hreal_on_top.txt

Deltat $=r * r *$ Deltau

Appending to SEOBNR/Hamiltonian-Hreal_on_top.txt

14.4 Step 13.d: $\Delta_{t}^{\prime}$ [Back to Section 0]

From the discussion after BB2010 Equation (5.47), we know that the prime notation indicates a derivative with respect to $r$. Using the definition of Section 14.3 , we have

$$
\Delta_{t}^{\prime}=2 r \Delta_{u}+r^{2} \Delta_{u}^{\prime}
$$

We define $\Delta_{u}$ in Section 14.5 and $r$ in Section 18.2 
[74]: \%\%writefile -a \$Ccodesdir/Hamiltonian-Hreal_on_top.txt

Deltatprm $=2 * r *$ Deltau $+r * r *$ Deltauprm

Appending to SEOBNR/Hamiltonian-Hreal_on_top.txt

14.5 Step 13.e: $\Delta_{u}$ [Back to Section 0]

From BB2010 Equation (5.73), we have

$$
\Delta_{u}=\bar{\Delta}_{u}[\underbrace{1+\eta \Delta_{0}+\eta \log (1+\operatorname{logarg})}_{\Delta_{u} \text { calibration term }}]
$$

We compute $\bar{\Delta}_{u}$ in Section 14.5 .1 and $\Delta_{u}$ calibration term and logarg in Section 14.5.2. From the discussion after BB2010 Equation (5.47), we know that primes denote derivatives with respect to $r$. We have

$$
\Delta_{u}=\bar{\Delta}_{u}^{\prime}\left(\Delta_{u} \text { calibration term }\right)+\bar{\Delta}_{u}\left(\Delta_{u} \text { calibration term }\right)^{\prime}
$$

[75]: \%\%writefile -a \$Ccodesdir/Hamiltonian-Hreal_on_top.txt

Deltauprm $=$ Deltaubarprm*Deltaucalib + Deltaubar $*$ Deltaucalibprm Deltau $=$ Deltaubar $*$ Deltaucalib

Appending to SEOBNR/Hamiltonian-Hreal_on_top.txt

14.5.1 Step 13.e.i: $\bar{\Delta}_{u}$ [Back to Section 0]

From BB2010 Equation (5.75), we have

$$
\bar{\Delta}_{u}=\frac{a^{2} u^{2}}{M^{2}}+\frac{1}{\eta K-1}\left(2 u+\frac{1}{\eta K-1}\right) .
$$

We define $a$ in Section 17.1, $u$ in Section 18.1, $M$ in Section 18.5, $\eta$ in Section 18.3, and $K$ in Section 14.5.4. From the discussion after BB2010 Equation (5.47), we know that primes denote derivatives with respect to $r$. We have

$$
\bar{\Delta}_{u}^{\prime}=\frac{-2 a^{2} u^{3}}{M^{2}}-\frac{2 u^{2}}{\eta K-1} .
$$

[76]: \%\%writefile -a \$Ccodesdir/Hamiltonian-Hreal_on_top.txt

Deltaubarprm $=-2 * \mathrm{a} * \mathrm{a} * \mathrm{u} * \mathrm{u} * \mathrm{u}-2 * \mathrm{u} * \mathrm{u} /($ etaKminus 1$)$ Deltaubar $=\mathrm{a} * \mathrm{a} * \mathrm{u} * \mathbf{u}+(2 * \mathrm{u}+1 /$ etaKminus 1$) /$ etaKminus 1

Appending to SEOBNR/Hamiltonian-Hreal_on_top.txt

14.5.2 Step 13.e.ii: $\Delta_{u}$ Calibration Term [Back to Section 0]

From BB2010 Equation (5.73), we have

$$
\begin{aligned}
\Delta_{u} \text { calibration term } & =1+\eta \Delta_{0}+\eta \log \left(1+\Delta_{1} u+\Delta_{2} u^{2}+\Delta_{3} u^{3}+\Delta_{4} u^{4}\right) \\
& =1+\eta\left[\Delta_{0}+\log \left(1+\Delta_{1} u+\Delta_{2} u^{2}+\Delta_{3} u^{3}+\Delta_{4} u^{4}\right)\right] .
\end{aligned}
$$

In T2014 Equation (2) an additional term is and is defined in Equation (A2) of this paper. We then have

$$
\Delta_{u} \text { calibration term }=1+\eta\left[\Delta_{0}+\log \left(1+\Delta_{1} u+\Delta_{2} u^{2}+\Delta_{3} u^{3}+\Delta_{4} u^{4}+\Delta_{5} u^{5}\right)\right] .
$$

In the LALSuite code itself (see LALSimIMRSpinEOBHamiltonianPrec.c line 274 on Git commit a70b43d), there's one more term ( $\left.\Delta_{5 \ell}\right)$, for which documentation is elusive. That bring us to

$$
\Delta_{u} \text { calibration term }=1+\eta\left[\Delta_{0}+\log (1+\underbrace{\Delta_{1} u+\Delta_{2} u^{2}+\Delta_{3} u^{3}+\Delta_{4} u^{4}+\Delta_{5} u^{5}+\Delta_{5} u^{5} \ln (u)}_{\text {logarg }})\right] .
$$

Note our notation for logarg. We define $u$ in Section 18.1, $\eta$ in Section 18.3, and the calibration coefficients $\Delta_{i}, i \in\{0,1,2,3,4\}$, in Section 14.5 .3 .

From the discussion after BB2010 Equation (5.47), we know that primes denote derivatives with respect to $r$. We have

$$
\left(\Delta_{u} \text { calibration term }\right)^{\prime}=\frac{-\eta u^{2}\left(\Delta_{1}+2 \Delta_{2} u+3 \Delta_{3} u^{2}+4 \Delta_{4} u^{3}+5 \Delta_{5} u^{4}+5 \Delta_{5 \ell} u^{4} \ln (u)+\Delta_{5 \ell} u^{5} u^{-1}\right)}{1+\log \arg } .
$$

[77]: \%/writefile -a \$Ccodesdir/Hamiltonian-Hreal_on_top.txt

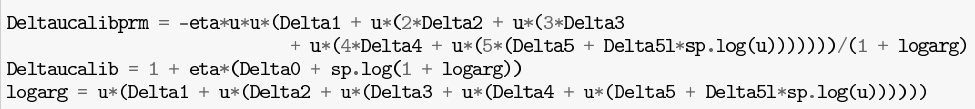


From BB2010 Equations (5.77)--(5.81), we have

$$
\begin{aligned}
\Delta_{0}= & K(\eta K-2) \\
\Delta_{1}= & -2(\eta K-1)\left(K+\Delta_{0}\right) \\
\Delta_{2}= & \frac{1}{2} \Delta_{1}\left(-4 \eta K+\Delta_{1}+4\right)-\frac{a^{2}}{M^{2}}(\eta K-1)^{2} \Delta_{0} \\
\Delta_{3}= & \frac{1}{3}\left[-\Delta_{1}^{3}+3(\eta K-1) \Delta_{1}^{2}+3 \Delta_{2} \Delta_{1}-6(\eta K-1)\left(-\eta K+\Delta_{2}+1\right)-3 \frac{a^{2}}{M^{2}}(\eta K-1)^{2} \Delta_{1}\right] \\
= & -\frac{1}{3} \Delta_{1}^{3}+(\eta K-1) \Delta_{1}^{2}+\Delta_{2} \Delta_{1}-2(\eta K-1)\left(\Delta_{2}-(\eta K-1)\right)-\frac{a^{2}}{M^{2}}(\eta K-1)^{2} \Delta_{1} \\
\Delta_{4}= & \frac{1}{12}\left\{6 \frac{a^{2}}{M^{2}}\left(\Delta_{1}^{2}-2 \Delta_{2}\right)(\eta K-1)^{2}+3 \Delta_{1}^{4}-8(\eta K-1) \Delta_{1}^{3}-12 \Delta_{2} \Delta_{1}^{2}+12\left[2(\eta K-1) \Delta_{2}+\Delta_{3}\right] \Delta_{1}\right. \\
& \left.+12\left(\frac{94}{3}-\frac{41}{32} \pi^{2}\right)(\eta K-1)^{2}+6\left[\Delta_{2}^{2}-4 \Delta_{3}(\eta K-1)\right]\right\} \\
\Delta_{5}= & (\eta K-1)^{2}\left\{\left(-\frac{4237}{60}+\frac{128}{5} \gamma+\frac{2275}{512} \pi^{2}-\frac{1}{3} a^{2}\left\{\Delta_{1}^{3}-3 \Delta_{1} \Delta_{2}+3 \Delta_{3}\right\}\right.\right. \\
& -\frac{\Delta_{1}^{5}-5 \Delta_{1}^{3} \Delta_{2}+5 \Delta_{1} \Delta_{2}^{2}+5 \Delta_{1}^{2} \Delta_{3}-5 \Delta_{2} \Delta_{3}-5 \Delta_{1} \Delta_{4}}{5(\eta K-1)^{2}} \\
& \left.\left.+\frac{\Delta_{1}^{4}-4 \Delta_{1}^{2} \Delta_{2}+2 \Delta_{2}^{2}+4 \Delta_{1} \Delta_{3}-4 \Delta_{4}}{2(\eta K-1)}+\frac{256}{5} \log (2)\right)+\frac{64}{5} \log (u)\right\} \\
\Delta_{5}= & \frac{64}{5}(\eta K-1)^{2} .
\end{aligned}
$$

We define $K$ in Section 14.5.4, $\eta$ in Section 18.3, $a$ in Section 17.1, and $M$ in Section 18.5. Note that the constant $\gamma$ is the Euler-Mascheroni, and the value is taken from the LALSuite documentation. In the Python code we donote $\gamma$ by EMgamma.

[78]: \%\%rritefile -a \$Ccodesdir/Hamiltonian-Hreal_on_top.txt

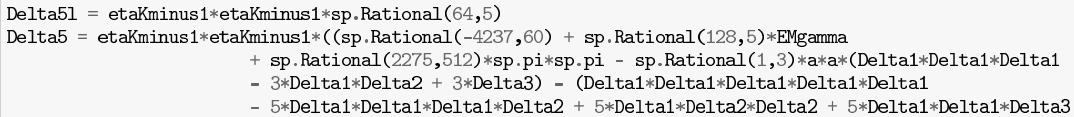

Appending to SEOBNR/Hamiltonian-Hreal_on_top.txt

14.5.4 Step 13.e.iv: $K$ [Back to Section 0]

The calibration constant $K$ is defined in T2014 Section II

$$
K=1.712-1.804 \eta-39.77 \eta^{2}+103.2 \eta^{3} .
$$

The term $\eta K-1$ is sufficiently common that we also define it:

$$
\text { etaKminus } 1=\eta K-1 .
$$

We define $\eta$ in Section 18.3 .

[79]: \%\%writefile -a \$Ccodesdir/Hamiltonian-Hreal_on_top.txt

etaKminus1 $=$ eta $* \mathrm{~K}-1$

$\mathrm{K}=1.712-1.803949138004582 *$ eta $-39.77229225266885 *$ eta*eta $+103.16588921239249 *$ eta*eta*eta

Appending to SEOBNR/Hamiltonian-Hreal_on_top.txt

14.6 Step 13.f: $\tilde{\omega}_{\mathrm{fd}}$ [Back to Section 0]

From BB2010 Equation (5.40), we have 


$$
\tilde{\omega}_{\mathrm{fd}}=2 a M r+\omega_{1}^{\mathrm{fd}} \eta \frac{a M^{3}}{r}+\omega_{2}^{\mathrm{fd}} \eta \frac{M a^{3}}{r} .
$$

From discussion after BB2010 Equation (6.7), we set $\omega_{1}^{\mathrm{fd}}=\omega_{2}^{\mathrm{fd}}=0$. Thus

$$
\tilde{\omega}_{\mathrm{fd}}=2 a M r
$$

We define $a$ in Section 17.1, $M$ in Section 18.5, and $r$ in Section 18.2 below.

[80]: \%\%writefile -a \$Ccodesdir/Hamiltonian-Hreal_on_top.txt

omegatilde $=2 * a * r$

Appending to SEOBNR/Hamiltonian-Hreal_on_top.txt

14.7 Step 13.g: $D^{-1}$ [Back to Section 0]

From BB2010 Equation (5.83),

$$
D^{-1}=1+\log \left[1+6 \eta u^{2}+2(26-3 \eta) \eta u^{3}\right] .
$$

We define $\eta$ in Section 18.3 and $u$ in Section 18.1 .

[81]: \%\%writefile -a \$Ccodesdir/Hamiltonian-Hreal_on_top.txt

Dinv $=1+\mathrm{sp} \cdot \log (1+6 *$ eta $* \mathbf{u} * \mathbf{u}+2 *(26-3 *$ eta $) *$ eta $* u * u * u)$

Appending to SEOBNR/Hamiltonian-Hreal_on_top.txt

15 Step 14: Terms Dependent on Coordinates [Back to Section 0]

We collect here terms directly dependend on the coordinates. See BB2010 Equations (4.5) and (4.6).

15.1 Step 14.a: $\Sigma$ [Back to Section 0]

From BB2010 Equation (4.5), we have

$$
\Sigma=r^{2}+a^{2} \cos ^{2} \theta
$$

We define $r$ in Section 18.2, $a$ in Section 17.1, and $\cos \theta$ in Section 15.4.

[82]: \%\%writefile -a \$Ccodesdir/Hamiltonian-Hreal_on_top.txt

Sigma $=r * r+a * a * \cos t h e t a * \cos$ theta

Appending to SEOBNR/Hamiltonian-Hreal_on_top.txt

15.2 Step 14.b: $\omega^{2}$ [Back to Section 0$]$

From BB2010 Equation (4.7),

$$
\omega^{2}=a^{2}+r^{2}
$$

We define $a$ in Section 17.1 and $r$ in Section 18.2.

[83]: \%\%ritefile -a \$Ccodesdir/Hamiltonian-Hreal_on_top.txt

$\mathrm{w} 2=\mathrm{a} * \mathrm{a}+\mathrm{r} * \mathrm{r}$

Appending to SEOBNR/Hamiltonian-Hreal_on_top.txt

15.3 Step 14.d: $\sin ^{2} \theta$ [Back to Section 0]

Using a common trigonometric idenitity,

$$
\sin ^{2} \theta=1-\cos ^{2} \theta .
$$

We define $\cos \theta$ in Section 15.4. Note that by construction (from discussion after BB2010 Equation (5.52)) 


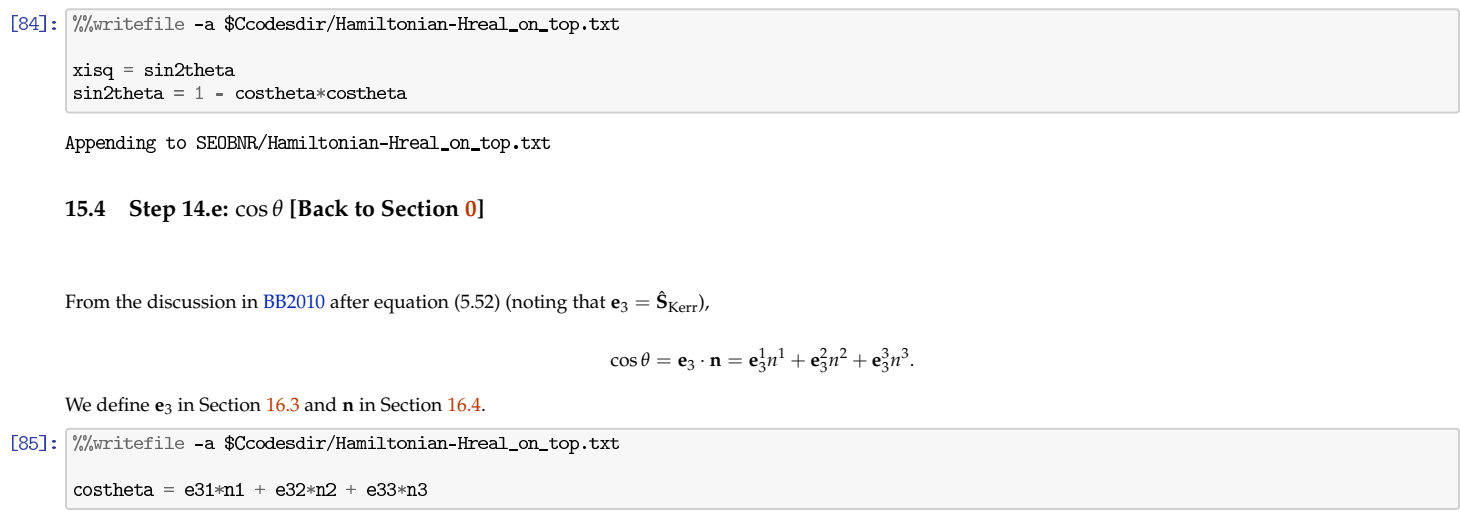

Appending to SEOBNR/Hamiltonian-Hreal_on_top.txt

16 Step 15: Important Vectors [Back to Section 0]

We collect the vectors common for computing $H_{\text {real }}$ (defined in Section 2) below.

16.1 Step 15.a: v [Back to Section 0]

From BB2010 Equation (3.39), we have

$$
\mathbf{v}=\mathbf{n} \times \boldsymbol{\xi}
$$

We define $\mathbf{n}$ in Section 16.4 and $\xi$ in Section 16.2.

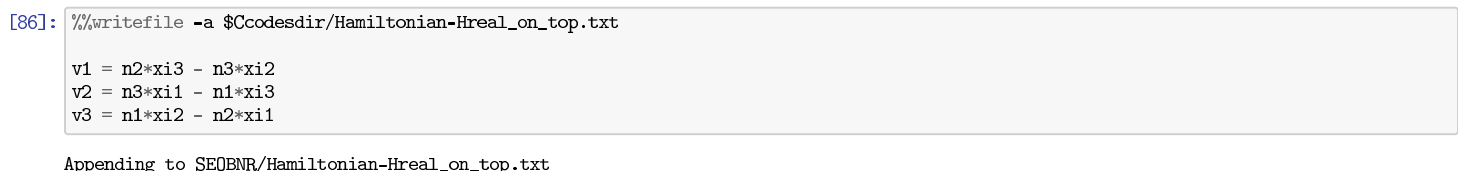

Appending to SEOBNR/Hamiltonian-Hreal_on_top.txt

16.2 Step 15.b: $\xi$ [Back to Section 0]

From BB2010 Equation (3.38), we have

We define $\mathbf{e}_{3}$ in Section 16.3 and $\mathbf{n}$ in Section 16.4

[87]: \%\%rritefile -a \$Ccodesdir/Hamiltonian-Hreal_on_top.txt

$\mathrm{xi} 1=\mathrm{e} 2 * \mathrm{n} 3-\mathrm{e} 3 * \mathrm{n} 2$
$\mathrm{xi2}=\mathrm{e} 1 * \mathrm{n} 3+\mathrm{e} 33 * \mathrm{n} 1$

$\mathrm{xi} 3=\mathrm{e} 31 * \mathrm{n} 2-\mathrm{e} 32 * \mathrm{n} 1$

Appending to SEOBNR/Hamiltonian-Hreal_on_top.txt

16.3 Step 15.c: $\mathbf{e}_{3}$ [Back to Section 0]

From the discussion in BB2010 after equation (5.52),

$$
\mathbf{e}_{3}=\hat{\mathbf{S}}_{\text {Kerr }} \text {. }
$$

We define $\hat{\mathbf{S}}_{\text {Kerr }}$ in Section 17.2.

[88]: \%\%writefile -a \$Ccodesdir/Hamiltonian-Hreal_on_top.txt

$$
\begin{aligned}
& \text { e31 }=\text { Skerrhat } 1 \\
& \text { e32 }=\text { Skerrhat2 } \\
& \text { e33 }=\text { Skerrhat3 }
\end{aligned}
$$

Appending to SEOBNR/Hamiltonian-Hreal_on_top.txt 
16.4 Step 15.d: $\mathrm{n}$ [Back to Section 0]

From BB2010 Equation (3.37), we have

$$
\mathbf{n}=\frac{\mathbf{x}}{r}
$$

where $\mathbf{x}=(x, y, z)$. We define $r$ in Section 18.2

[89]: \%\%ritefile -a \$Ccodesdir/Hamiltonian-Hreal_on_top.txt

$\mathrm{n} 1=\mathrm{x} / \mathrm{r}$

$\mathrm{n} 2=\mathrm{y} / \mathrm{r}$

Appending to SEOBNR/Hamiltonian-Hreal_on_top.txt

17 Step 16: Spin Combinations $\sigma, \sigma^{*}$, and $\mathrm{S}_{\text {Kerr }}$ [Back to Section 0]

We collect here various combinations of the spins.

17.1 Step 16.a: $a$ [Back to Section 0]

From BB2010 Equation (4.9), we have

$$
a=\frac{\left|\mathbf{S}_{\text {Kerr }}\right|}{M} .
$$

We define $\left|\mathbf{S}_{\text {Kerr }}\right|$ in Section 17.3 and $M$ in Section 18.5.

[90]: \%\%ritefile -a \$Ccodesdir/Hamiltonian-Hreal_on_top.txt

$\mathrm{a}=$ Skerrmag

Appending to SEOBNR/Hamiltonian-Hreal_on_top.txt

17.2 Step 16.b: $\hat{\mathrm{S}}_{\text {Kerr }}$ [Back to Section 0]

From BB2010 Equation (4.24), we have

$$
\hat{\mathbf{S}}_{\text {Kerr }}=\frac{\mathbf{S}_{\text {Kerr }}}{\left|\mathbf{S}_{\text {Kerr }}\right|}
$$

We define $\mathbf{S}_{\text {Kerr }}$ in Section 17.4.

[91]: \%\%writefile -a \$Ccodesdir/Hamiltonian-Hreal_on_top.txt

Skerrhat $1=$ Skerr $1 /$ Skerrmag

Skerrhat2 $=$ Skerr2/Skerrmag

Skerrhat3 $=$ Skerr3 $/$ Skerrmag

Appending to SEOBNR/Hamiltonian-Hreal_on_top.txt

17.3 Step 16.c: $\left|S_{\text {Kerr }}\right|$ [Back to Section 0]

We have

$$
\left|\mathbf{S}_{\text {Kerr }}\right|=\sqrt{\mathbf{S}_{\text {Kerr }}^{1} \mathbf{S}_{\text {Kerr }}^{1}+\mathbf{S}_{\text {Kerr }}^{2} \mathbf{S}_{\text {Kerr }}^{2}+\mathbf{S}_{\text {Kerr }}^{3} \mathbf{S}_{\text {Kerr }}^{3}}
$$

We define $\mathbf{S}_{\text {Kerr }}$ in Section 17.4

[92]: \%\%writefile -a \$Ccodesdir/Hamiltonian-Hreal_on_top.txt

Skerrmag $=$ sp.sqrt $($ Skerr $1 *$ Skerr1 $1+$ Skerr2*Skerr2 + Skerr $3 *$ Skerr3 $)$

Appending to SEOBNR/Hamiltonian-Hreal_on_top.txt

17.4 Step 16.d: $\mathrm{S}_{\text {Kerr }}$ [Back to Section 0]

From BB2010 Equation (5.64):

$$
\mathbf{S}_{\mathrm{Kerr}}=\sigma+\frac{1}{c^{2}} \boldsymbol{\Delta}_{\sigma}
$$


In BB2010 Equation (5.67), $\Delta_{\sigma}=0$. Thus

We define $\sigma$ in Section 17.5.

[93]: \%\%ritefile -a \$Ccodesdir/Hamiltonian-Hreal_on_top.txt

Skerr1 $=$ sigma1

Skerr2 $=$ sigma2

Skerr3 $=$ sigma 3

Appending to SEOBNR/Hamiltonian-Hreal_on_top.txt

17.5 Step 16.e: $\sigma$ [Back to Section 0]

From BB2010 Equation (5.2):

$$
\sigma=\mathbf{S}_{1}+\mathbf{S}_{2}
$$

[94]: \%\%ritefile -a \$Ccodesdir/Hamiltonian-Hreal_on_top.txt

sigma1 $=\mathrm{S} 1 \mathrm{x}+\mathrm{S} 2 \mathrm{x}$

sigma2 $=$ S1y + S2y

sigma3 $=\mathrm{S} 1 \mathrm{z}+\mathrm{S} 2 \mathrm{z}$

Appending to SEOBNR/Hamiltonian-Hreal_on_top.txt

17.6 Step 16.f: $\sigma^{*}$ [Back to Section 0]

From BB2010 Equation (5.3):

$$
\sigma^{*}=\frac{m_{2}}{m_{1}} \mathbf{S}_{1}+\frac{m_{1}}{m_{2}} \mathbf{S}_{2}
$$

[95]: \%\%ritefile -a \$Ccodesdir/Hamiltonian-Hreal_on_top.txt

sigmastar $1=\mathrm{m} 2 / \mathrm{m} 1 * \mathrm{~S} 1 \mathrm{x}+\mathrm{m} 1 / \mathrm{m} 2 * \mathrm{~S} 2 \mathrm{x}$

sigmastar $2=\mathrm{m} 2 / \mathrm{m} 1 * \mathrm{~S} 1 \mathrm{y}+\mathrm{m} 1 / \mathrm{m} 2 * \mathrm{~S} 2 \mathrm{y}$ sigmastar $3=\mathrm{m} 2 / \mathrm{m} 1 * \mathrm{~S} 1 \mathrm{z}+\mathrm{m} 1 / \mathrm{m} 2 * \mathrm{~S} 2 \mathrm{z}$

Appending to SEOBNR/Hamiltonian-Hreal_on_top.txt

18 Step 17: Fundamental Quantities [Back to Section 0]

We collect here fundamental quantities from which we build $H_{\text {real }}$ (defined in Section 2).

18.1 Step 17.a: $u$ [Back to Section 0]

From the discussion after BB2010 Equation (5.40),

$$
u=\frac{M}{r} .
$$

We define $M$ in Section 18.5 and $r$ in Section 18.2.

[96]: \%\%writefile -a \$Ccodesdir/Hamiltonian-Hreal_on_top.txt

$\mathrm{u}=1 / \mathrm{r}$

Appending to SEOBNR/Hamiltonian-Hreal_on_top.txt

18.2 Step 17.b: $r$ [Back to Section 0]

From the discussion after BB2010 Equation (5.52),

$$
r=\sqrt{x^{2}+y^{2}+z^{2}}
$$

[97]: \%\%writefile -a \$Ccodesdir/Hamiltonian-Hreal_on_top.txt

$r=\operatorname{sp} \cdot \operatorname{sqrt}(x * x+y * y+z * z)$

Appending to SEOBNR/Hamiltonian-Hreal_on_top.txt 
From the discussion preceding BB2010 Equation (5.1),

$$
\eta=\frac{\mu}{M}
$$

We define $\mu$ in Section 18.4 .

[98]: \%\%writefile -a \$Ccodesdir/Hamiltonian-Hreal_on_top.txt

eta $=\mathrm{mu} / \mathrm{M}$

Appending to SEOBNR/Hamiltonian-Hreal_on_top.txt

\subsection{Step 17.d: $\mu$ [Back to Section 0]}

From the discussion preceding BB2010 Equation (5.1),

$$
\mu=\frac{m_{1} m_{2}}{M} .
$$

We define $M$ in Section 18.5

[99]: \%\%writefile -a \$Ccodesdir/Hamiltonian-Hreal_on_top.txt

$\mathrm{mu}=\mathrm{m} 1 * \mathrm{~m} 2 / \mathrm{M}$

Appending to SEOBNR/Hamiltonian-Hreal_on_top.txt

18.5 Step 17.e: $M$ [Back to Section 0]

From the discussion preceding BB2010 Equation (5.1),

$$
M=m_{1}+m_{2} .
$$

[100]: \%\%writefile -a \$Ccodesdir/Hamiltonian-Hreal_on_top.txt

$\mathrm{M}=\mathrm{m} 1+\mathrm{m} 2$

Appending to SEOBNR/Hamiltonian-Hreal_on_top.txt

\section{Step 18: Validation [Back to Section 0]}

The following code cell reverses the order of the expressions output to SEOBNR/Hamiltonian_on_top.txt and creates a Python function to validate the value of $H_{\text {real }}$ against the SEOBNRv3 Hamiltonian value computed in LALSuite git commit bba40f21e9 for command-line input parameters

-M 23 -m 10 -f 20 -X 0.01 -Y 0.02 -Z -0.03 -x 0.04 -y - 0.05 -z 0.06.

[101]: import numpy as np

import difflib, sys, os

\# The subterms in the Hamiltonian expression are sometimes written on more than

\# one line for readability in this Jupyter notebook. We first create a file of

\# one-line expressions, Hamiltonian-Hreal_one_line_expressions.txt.

with open(os.path.join(Ccodesdir,"Hamiltonian-Hreal_one_line_expressions.txt"), "w") as output:

count $=0$

\# Read output of this notebook

for line in list(open("SEOBNR/Hamiltonian-Hreal_on_top.txt")) :

\# Read the first line

f count $=0$ :

prevline=line

\#Check if prevline is a complete expression

elif "=" in prevline and "=" in line:

output.write("\%s\n" \% prevline.strip('\n'))

prevline=line

\# Check if line needs to be adjoined to prevline

elif "=" in prevline and not " $="$ in line:

prevline = prevline.strip (' $\backslash n$ ')

prevline $=$ (prevline+line) $\cdot$ replace $("$ ","'")

\# Be sure to print the last line.

if count $=$ len(list(open("SEOBNR/Hamiltonian-Hreal_on_top.txt")))-1:

if not "=" in line:

print("ERROR. Algorithm not robust if there is no equals sign on the final line. Sorry.") sys.exit (1)

else:

output.write( $(1 \%$ s" \% line) 
count $=$ count +1

\# Now reverse the expressions and urite them in a function

\# This formulation is used to check that we get a reasonable $H_{-}$real value

with open(os.path.join(Ccodesdir,"Hreal_on_bottom.py"), "w") as output:

output.write("import numpy as np\ndef compute_Hreal (m1=23., m2=10., EMgamma=0.577215664901532860606512090082402431, tortoise=1, L

$\mathrm{dSO}=-7.966696593617955 \mathrm{e}+01, \quad \mathrm{SS}=1.261873764525631 \mathrm{e}+01, \mathrm{x}=2.129681018601393 \mathrm{e}+01, \mathrm{y}=0.000000000000000 \mathrm{e}+00, \mathrm{z}=0.0000000000000000 \mathrm{e}+00$,

$\mathrm{p} 1=0.000000000000000 \mathrm{e}+00, \mathrm{p} 2=2.335391115580442 \mathrm{e}-01, \mathrm{p} 3=-4.235164736271502 \mathrm{e}-22, \mathrm{~S} 1 \mathrm{x}=4.857667584940312 \mathrm{e}-03, \mathrm{~S} 1 \mathrm{y}=9.715161660389764 \mathrm{e}-03$,

$\mathrm{S} 1 \mathrm{z}=-1.457311842632286 \mathrm{e}-02, \mathrm{~S} 2 \mathrm{x}=3.673094582185491 \mathrm{e}-03, \mathrm{~S} 2 \mathrm{y}=-4.591302628615413 \mathrm{e}-03, \mathrm{~S} 2 \mathrm{z}=5.509696538546906 \mathrm{e}-03): \mathrm{nn})$

for line in reversed(list(open("SEOBNR/Hamiltonian-Hreal_one_line_expressions.txt"))):

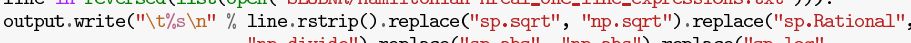

"np.divide"). replace("sp.abs", "np.abs").replace("sp.log", "np.log") .replace("sp.sign", "np.sign") .replace("sp.pi",

output.write("\treturn Hreal") "np.pi"))

\# Now reverse the expressions in a standalone text file

\# This formulation is used as a harsher validation check that all expressions agree with a trusted list

with open(os.path.join(Ccodesdir,"Hamiltonian_expressions.txt-VALIDATION"), "w") as output:

for line in reversed(list(open("SEOBNR/Hamiltonian-Hreal_one_line_expressions.txt"))):

output.write("\t\%s\n" \% line.rstrip().replace("sp.sqrt", "np.sqrt").replace("sp.Rational",

"np.divide").replace("sp.abs", "np.abs").replace("sp.log",

"np.log").replace("sp.sign", "np.sign").replace("sp.pi",

"np.pi"))

print("Printing difference between notebook output and a trusted list of expressions...")

\# Open the files to compare

file = "Hamiltonian_expressions.txt"

outfile = "Hamiltonian_expressions.txt-VALIDATION"

print("Checking file " + outfile)

with open(os.path.join(Ccodesdir,file), "r") as file1, open(os.path.join(Ccodesdir,outfile), "r") as file2:

\# Read the lines of each file

file1 lines = file1, readlines ()

file2 lines $=$ file2 readlines ()

num_diffs $=0$

for line in difflib.unified_diff(file1_lines, file2_lines, fromfile=os.path.join(Ccodesdir,file), tofile=os.path.

join(Ccodesdir, outfile)):

sys.stdout.writelines(line)

num_diffs $=$ num_diffs +1

if num_diffs $=0$ :

print ("No difference. TEST PASSED!")

else:

print("ERROR: Disagreement found with the trusted file. See differences above.")

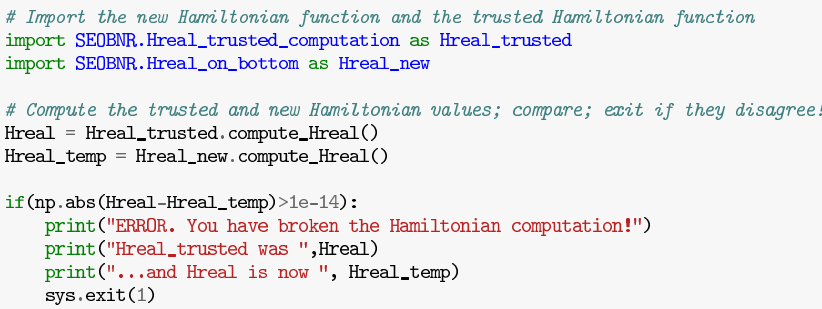

Printing difference between notebook output and a trusted list of expressions...

Checking file Hamiltonian_expressions.txt-VALIDATION

No difference. TEST PASSED!

\section{Step 19: Output this notebook to BTEX-formatted PDF file [Back to Section 0]}

The following code cell converts this Jupyter notebook into a proper, clickable $\mathrm{LT}_{\mathrm{E}} \mathrm{X}$-formatted PDF file. After the cell is successfully run, the generated PDF may be found in the root NRPy+ tutorial directory, with filename Tutorial-SEOBNR_Documentation.pdf (Note that clicking on this link may not work; you may need to open the PDF file through another means.)

[102]: !jupyter nbconvert --to latex --template latex_nrpy_style.tplx --log-level='WARN' Tutorial-SEOBNR_Documentation.ipynb !pdflatex -interaction=batchmode Tutorial-SEOBNR_Documentation.tex

! pdflatex -interaction=batchmode Tutorial-SEOBNR_Documentation.tex

!pdflatex -interaction=batchmode Tutorial-SEOBNR Documentation tex

!rm -f Tut*. out Tut*. aux Tut*.log

This is pdfTeX, Version 3.14159265-2.6-1.40.18 (TeX Live 2017/Debian) (preloaded format=pdflatex)

restricted \write18 enabled.

entering extended mode

This is pdfTeX, Version 3.14159265-2.6-1.40.18 (TeX Live 2017/Debian) (preloaded

format=pdflatex

restricted \write18 enabled.

entering extended mode

This is pdfTeX, Version 3.14159265-2.6-1.40.18 (TeX Live 2017/Debian) (preloaded

format=pdflatex) 
restricted \write18 enabled.

entering extended mode

[ ]: 


\section{Appendix B}

The following pages contain a copy of the eigenframe approach documentation Jupyter notebook output as a PDF for easy reading. Note that the notebook has been output in landscape mode and that two pages have been included per page of this dissertation. 


\section{Tutorial-BigG_Image_Analysis}

April 29, 2020

\section{-1 The Eigenframe Approach to Image Analysis}

-1.1 Author: Tyler Knowles

-1.1.1 This module documents the eigenframe approach to image analysis, developed originally with Dr. Zachariah B. Etienne of West Virginia University and Dr. Brian D'Urso and Charlie Lewandowski of Montana State University for determining the position of a microsphere suspended in a magnetic trap. See Lewandowski et. al. (accelerometer) and Lewandowski et. al. (tilt stabilization).

-1.2 Introduction

\section{-1.2.1 Motivation}

Newton's gravitational constant $G$ is one of the most poorly measured fundamental constants of physics. Several experiments in the last few decades have found values of $G$ separated by as many as ten standard deviations (see the figure here). The consensus is that this discrepancy is due to poorly understood systematics in the apparatus.

Dr. Brian D'Urso postulated that the systematic uncertainties of a microsphere suspended in a magentic trap would be better understood than the uncertainties in a torsion pendulum, the apparatus on which many measurements of $G$ depend. The accuracy of the measurement obtained from this microsphere apparatus depends on accurate tracking of the microsphere. That is, our data is a set of equally-spaced-in-time images of the microsphere (which never leaves the field of view), and we must find the discplacement of the microsphere with respect to some fiducial (usually initial) position.

Determining the translation between two similar images is a well-understood problem and there exist many solutions that allow for subpixel resolution (see, e.g. Guizar-Sicairos, Thurman, and Fienup (2008)). We found that by using the register_translation() function of scikit-image produced jumps in what should be a smooth particle position function. We attributed these jumps to noise in the background of the initial image in the data set, against which we correlated al other frames. We deveoloped the following algorithm for smoothing out this noise: 1. estimate the particle position with register_translation(), relative to the first image in the data set; 1 . use this position information to overlay all images in the frequency domain onto the first image and perform a global average; and 1. translate this averaged frame ("eigenframe") to each frame in the data set and correlate to refine the position estimate obtained in step 1 .

Note that we can iterate this process (repeating steps 2 and 3) as many times as we would like. We iterated until the standard deviation in differences between subsequent $z$-position values dropped below the readout noise level of the camera used to take the images.

In pseudocode:

eigenframe $=$ reference_image

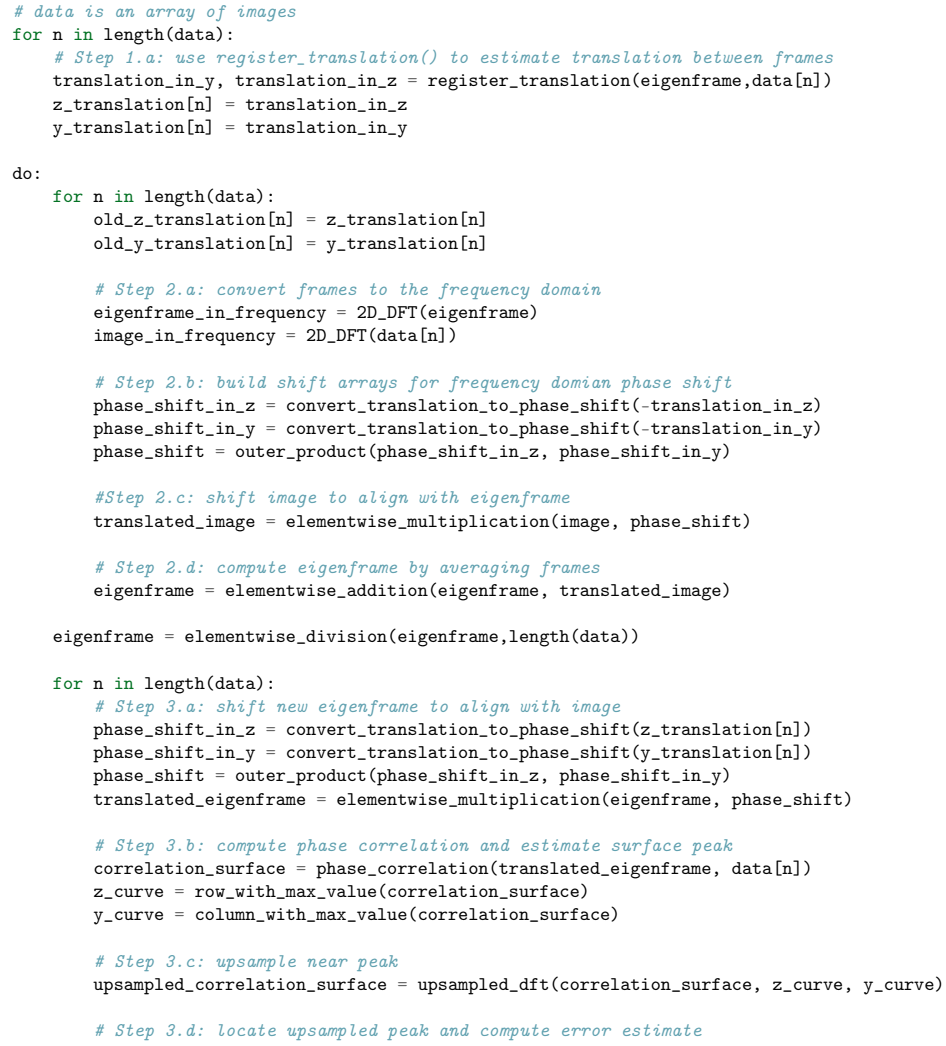


$z_{-}$curve $=$row_with_max_value (upsampled_correlation_surface)

y_curve = column_with_max_value (upsampled_correlation_surface)

refined_translation_in_z $=$ locate_peak (z_curve)

refined_translation_in_y $=$ locate_peak (y_curve)

\# Step 3.e: refine the translation values

$z_{-}$translation $[\mathrm{n}]=\mathrm{z}_{-}$translation $[\mathrm{n}]+$ refinend_translation_in_z

y_translation $[\mathrm{n}]=\mathrm{y}_{-}$translation $[\mathrm{n}]+$ refinend_translation_in_y

while(standard_deviation(old_z_translation - z_translation) > readout noise)

This notebook documents this "iterative eigenframe process" using two 256x128 pixel sample images (the 1st and 5000th) from the data set data_2020_01_16/unstabilized_2, provided by Charlie Lewandowski.

\section{Table of Contents}

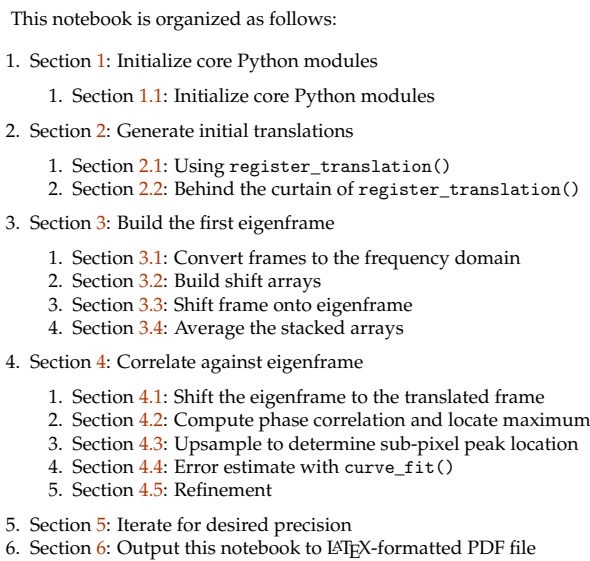

\section{Step 0: Initialize core Python modules [Back to Section 0]}

Let's start by importing all needed modules from Python and defining the quadratic function for error estimates.

\subsection{Step 0.a: Initialize core Python modules [Back to Section 0]}

Let's start by importing all needed modules from Python. We check for installations of SciPy and scikit-feature, from which we will use curve_fit() and register_translation(), repsectively. We also import the function_upsampled_dft directly from the register_translation source code. This function provides an efficient way to compute (interpolate) the correlation surface near the peak for subpixel resolution (see the source code for a description).

[1]: !pip install scikit-image

!pip install scipy

import numpy as $\mathrm{np}$

import matplotlib.pyplot as plt

import matplotlib.pyplot as

from skimage.feature import register translation

from skimage.feature.register_translation import _upsampled_dft

Requirement already satisfied: scikit-image in

/Users/K/juppy3/lib/python3.5/site-packages $(0.15 .0)$

Requirement already satisfied: imageio $>=2.0 .1$ in

/Users/K/juppy3/lib/python3.5/site-packages (from scikit-image) (2.8.0)

Requirement already satisfied: scipy $>=0.17 .0$ in

/Users/K/juppy3/lib/python3.5/site-packages (from scikit-image) (1.3.0)

Requirement already satisfied: matplotlib! $=3.0 .0,>=2.0 .0$ in

Users/K/juppy3/lib/python3.5/site-packages (from scikit-image) (3.0.3)

Requirement already satisfied: networkx $>=2.0$ in

/Users/K/juppy3/lib/python3.5/site-packages (from scikit-image) (2.4)

Requirement already satisfied: PyWavelets $>0.4 .0$ in

/Users/K/juppy3/lib/python3.5/site-packages (from scikit-image) (1.1.1)

Requirement already satisfied: pillow $>=4.3 .0$ in

/Users/K/juppy3/lib/python3.5/site-packages (from scikit-image) (7.1.1)

Requirement already satisfied: numpy in /Users/K/juppy3/lib/python3.5/site-

packages (from imageio $>=2.0 .1$->scikit-image) (1.16.4)

Requirement already satisfied: python-dateutil $>=2.1$ in

/Users/K/juppy3/lib/python3.5/site-packages (from

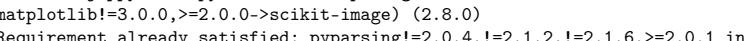

equireme already satisfied: pyparsing $=2.0 .4, !$

Users

Requirement already satisfied: cycler $>=0.10$ in

/Users/K/juppy3/lib/python3.5/site-packages (from 
matplotlib! $=3.0 .0,>=2.0 .0$ - >scikit-image $)(0.10 .0)$

Requirement already satisfied: kiwisolver $>=1.0 .1$ in

Users/K/juppy3/lib/python3.5/site-packages (from

matplotlib! $=3.0 .0,>=2.0 .0$ - >scikit-image $)(1.1 .0)$

Requirement already satisfied: decorator $>=4.3 .0$ in

/Users/K/juppy3/lib/python3.5/site-packages (from networkx >=2.0->scikit-image)

$(4.4 .0)$

Requirement already satisfied: six $>=1.5$ in /Users/K/juppy3/lib/python3.5/site-

packages (from python-dateutil $>=2.1$ - $>$ matplotlib $!=3.0 .0,>=2.0 .0->$ scikit-image)

$(1.12 .0)$

Requirement already satisfied: setuptools in /Users/K/juppy3/lib/python3.5/site

packages (from kiwisolver $>=1.0 .1$->matplotlib! $=3,0.0,>=2.0 .0$ - $>$ scikit-image)

(41.0.1)

NARNING: You are using pip version 19.1.1, however version 20.0 .2 is

ayailable

You should consider upgrading via the 'pip install --upgrade pip' command.

Requirement already satisfied: scipy in /Users/K/juppy3/lib/python3.5/site-

packages (1.3.0)

Requirement already satisfied: numpy $>=1.13 .3$ in

/Users/K/juppy3/lib/python3.5/site-packages (from scipy) (1.16.4)

NARNING: You are using pip version 19.1 .1 , however version 20.0 .2 is

available.

Vou should consider upgrading via the 'pip install --upgrade pip' command.

\section{Step 1: Generate initial translations [Back to Section 0]}

We first use register_translation to estimate the microsphere's translation relative to its initial position (that is, its position in the first frame). Below we show how to use register_translation(), and then give an overview of the steps taken by register_translation().

2.1 Step 1.a: Using register_translation() [Back to Section 0]

Our data was provided as a raw data file, so individual images are read byte-by-byte. Each pixel is composed of a 12-bit integer stored in two bytes (so pixel values range from 0 to $\left.2^{12}-1=4095\right)$. Throughout out code, we use $\mathrm{Nz}$ to denote the number of pixels in the horizontal $(z)$ direction and Ny to denote the number of pixels in the vertical $(y)$ direction. Our two sample images are saved as sample_data_1 and sample_data_5000. We also use upsample_factor=1000 so that register_translation() returns translation values to $\frac{1}{1000}$ of a pixel.

[2]: \# Load the images

image 1 = "sample_data $1 "$

image_2 = "sample_data_5000"

\# Set constants used throughout

$\mathrm{Nz}=256$

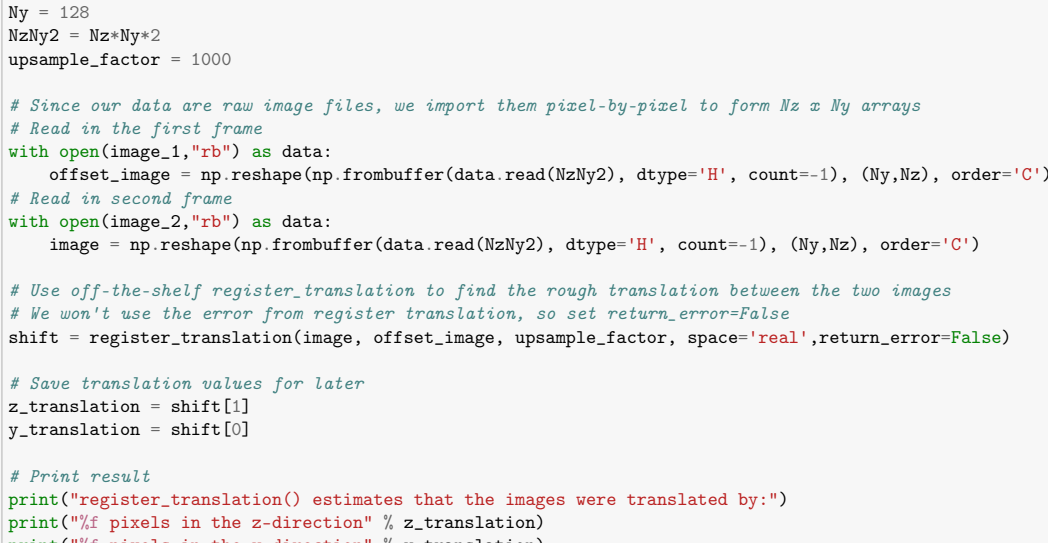

\subsection{Step 1.b: Behind the scenes [Back to Section 0]}

This code shows what is happening inside the register_translation() routine, and is lightly edited from the example here. The general formula for finding the translation between two images can be found at https://en.wikipedia.org/wiki/Phase_correlation.

Take a moment to consider the two raw images (shown below), and consider the microsphere's translation in the $z$-direction. The microsphere begins at about the middle of the image, meaning it's center has z-coordinate approximately at pixel 128 (half-way across the image of 256 pixels wide). In the later image, it appears to have moved about half-way to the right edge; that is translated probably $\frac{128}{2}=64$ pixels to the right. Above, we found that the $z$-translation was about 58 pixels so our intuition gave a reasonable estimate.

In the cross-correlation image, the peak (in yellow) is about 64 pixels to the right of the left edge of the image. That indicates the particle has moved about 64 pixels to the right. The peak is also split between the top and bottom of the frame, indicating that the microsphere did not move much in the vertical direction (which is also reasonable by eye). Note that the correlation surface is periodic in both the horizontal and vertical directions.

Note also that register_translation() outputs the translation array in format $(y, z)$. 
[3]: \# First plot the two images

fig $=$ plt.figure $($ figsize $=(16,8))$

ax1 $=$ plt. $\operatorname{subplot}(1,3,1)$

$\mathrm{ax2}=$ plt. subplot $(1,3,2$, sharex $=\mathrm{ax} 1$, sharey $=\mathrm{ax} 1)$

ax3 $=$ plt. $\operatorname{subplot}(1,3,3)$

ax1. imshow (offset_image, cmap='gray')

ax1.set_axis_off ()

ax1.set_title('Reference image')

ax2. imshow (image, $c$ map $=$ ' gray')

ax2. set_axis_off ()

ax2.set_title('Translated image')

\# Compute the cross-correlation surface, found using the formula at

\# https://en.wikipedia.org/wiki/Phase_correlation

\# but ignoring normalization

image_product $=$ np.fft.fft2(image) $*$ np.fft.fft2(offset_image) $\cdot \operatorname{conj}()$

\# Display the cross-correlation surface

cc_image $=n p$. fft. ifft2(image_product)

ax3. imshow (cc_image. real)

ax3. set_axis_off ()

ax3. set_title("Cross-correlation")

plt. show()

\# Print shift calculated in Step 1.6 for comparison with images

print ("Detected offset $(y, z): "$, shift)

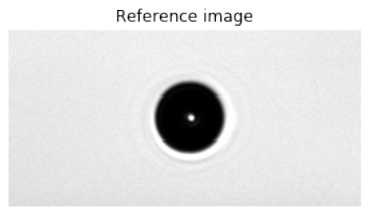

Translated image

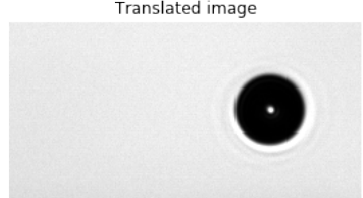

Cross-correlation

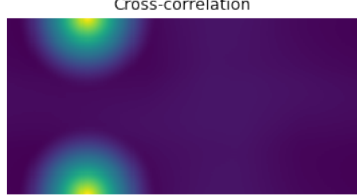

Detected offset $(y, z):[0.10557 .725]$

\section{Step 2: Build an eigenframe [Back to Section 0]}

Next we describe how eigenframes are constructed. During the first correlation (above), we defaulted to correlating against the first frame (to which we refe as the "zeroth eigenframe"). Now we overlay subsequent frames onto this frame in the frequency domain. Translations in the time domain are phase shifts in the frequency domain, and the shift arrays were based on this Matlab example.

We note that a similar process may be done in the time domain rather that in the frequency domain. We found, however, that building an eigenframe in the time domain resulted in large errors with (nearly) half-pixel translations. This is somewhat intuitive in the time domain, since we must interpolate midway between two pixels to determine the translated pixel value. In the frequency domain, the issue hinges on the fact the highest frequency component is a purely real number (since the discrete Fourier transform is even symmetric). A half-pixel translation converts this frequency component into a purely imaginary number, the information of which is lost when converting an inverse Fourier transform. As such, we build the eigenframe in the frequency domain and then correlate against it (in step 3) without transforming it into the time domain.

\subsection{Step 2.a: Convert frames to the frequency domain [Back to Section 0]}

We first convert the zeroth eigenframe and the translated frame to the frequency domain.

[4]: \# The previous eigenframe is frame

eigenframe in $_{-}$time $=$offset_image

\# FFT the eigenframe to get into frequency domain

eigenframe_in_freq $=$ np.fft.fft2 $($ eigenframe_in_time $)$

\# Read in second frame for averaging

frame_in_time $=$ image

\# FFT the eigenframe for averaging

frame_in_freq $=n p . f f t . f f t 2($ frame_in_time $)$

\subsection{Step 2.b: Build shift arrays [Back to Section 0]}

To shift the translated image onto the zeroth eigenframe, we build $z$ and $y$ shift arrays encoding translation in the frequency domain. We then take the outer product of these arrays to combine the two shifts into one array.

In one dimension, suppose that $h(x)=f\left(x-x_{0}\right)$ represents a translation by $x_{0}$ of the function $f$. Then the Fourier transform of $h, \mathcal{F}_{h}$, is given by

$$
\mathcal{F}_{h}(\xi)=e^{-2 \pi i x_{0} \xi} \mathcal{F}_{f}(\xi) .
$$

(see https://en.wikipedia.org/wiki/Fourier_transform\#Translation_/_time_shifting). The $z$ and $y$ shift arrays contain the exponential coefficient $e^{-2 \pi i x_{0} \tilde{\xi}}$, where $x_{0}$ is the translation found in Step 1.a and $\xi$ is the pixel number shifted so that $\xi \in\left[-\frac{N}{2}, \frac{N}{2}\right)$ (where $N$ is the number of pixels in the dimension under consideration).

[5]: \# Initialize shift arrays

shift_in_z $=[]$ 
\# No negative in $\exp ()$ since we're shifting *back* to the first frame

\# The if condition is used to match the array format in the Matlab code

\# which takes symmetry into account:

\# https://en.wikipedia.org/wiki/Discrete_Fourier_transform\#DFT_of_real_and_purely_imaginary_signals

Note also that Matlab starts index arrays at 1 rather than

for $\mathrm{y}$ in range(Ny):

if $\mathrm{y}<\mathrm{Ny} / 2$ :

shift_in_y. append (np. exp (2.*np. pi $*$ np . complex $\left.(0,1) * y_{\text {_translation } *(y / N y))}\right)$ else:

shift_in_y. append (np. exp $\left(2 . * n p . p i * n p . \operatorname{complex}(0,1) * y_{-}\right.$translation $\left.\left.*((y-N y) / N y)\right)\right)$

shift_in_y = np array (shift_in_y)

conjugate_index $=$ int $(\mathrm{Ny} / 2)$

shift_in_y [conjugate index$_{-}=\mathrm{np} \cdot \mathrm{real}\left(\mathrm{shift}_{-} \mathrm{in}_{-} \mathrm{y}[\right.$ conjugate _index $)$

for $z$ in range $(\mathrm{Nz})$

if $z<N z / 2$ :

else:

(n)

shift_in_z append (np. exp $\left(2, * n p\right.$. pi $*$ np. complex $\left.\left.(0,1) * z_{\text {_translation }} *((\mathrm{z}-\mathrm{Nz}) / \mathrm{Nz})\right)\right)$

shift_in_z $=$ np.array (shift_in_z)

conjugate_index $=$ int $(\mathrm{Nz} / 2)$

shift_in_z [conjugate_index] $=n p \cdot r e a l($ shift_in_z $[$ conjugate_index] $)$

\# To combine the 1-dimentional translation we use an outer product

shift_all $=$ np.outer $($ shift_in_y, shift_in_z)

3.3 Step 2.c: Shift frame onto eigenframe [Back to Section 0]

Shifting the translated frame onto the eigenframe is a matrix product operation in the frequency domain

[6]: \# Create the phase-shifted frame

shifted_frame $=$ np.multiply $($ frame_in_freq, shift_all $)$

\subsection{Step 2.d: Average the stacked arrays [Back to Section 0]}

Add the values of the shifted frame to the eigenframe and then average each element of the array. We plot the imaginary parts of the frames; note that important features of each image are only visible near the corners of the frames.

[13]: \# First plot the real part of the frequency-domain images

fig $=$ plt.figure $(f i g s i z e=(16,8))$

ax1 $=$ plt.subplot $(1,2,1)$

ax2 $=$ plt $\cdot \operatorname{subplot}(1,2,2)$

$\# a x 3=p l t . \operatorname{subplot}(2,2,3)$

ax1. imshow (eigenframe_in_freq. imag, cmap="Accent")

ax1. set_axis_off ()

ax1.set_title('Eigenframe in frequency')

ax2 . imshow (shifted_frame . imag, cmap="Accent" )

ax2. set_axis_off ()

ax2. set_title('Translated image in frequency')

plt. show ()

\# Build the average frame by adding values elementwise: this "+=" operation is a NumPy array operation

eigenframe_in_freq $+=$ shifted_frame

\#Take average of each pixel for eigenframe

eigenframe_in_freq $/=$

\# Show the output of the averaged frame

fig $=$ plt.figure $($ figsize $=(9,5))$

ax1 $=$ plt. $\operatorname{subplot}(1,1,1)$

im $=$ ax1.imshow (eigenframe_in_freq.imag, cmap="Accent")

ax1.set_axis_off ()

ax1.set_title("New eigenframe")

plt. colorbar (im, shrink $=0.8$ )

plt. show (

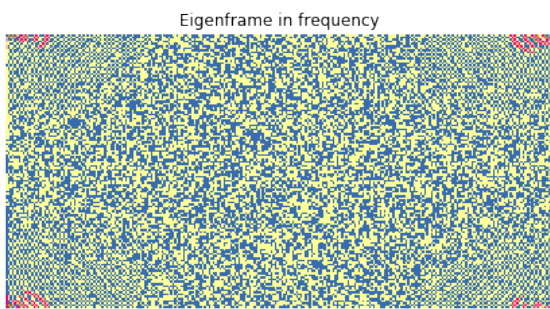

Translated image in frequency

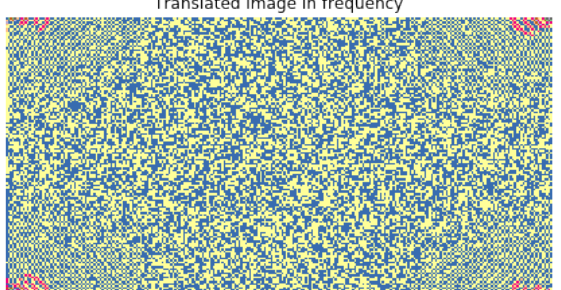




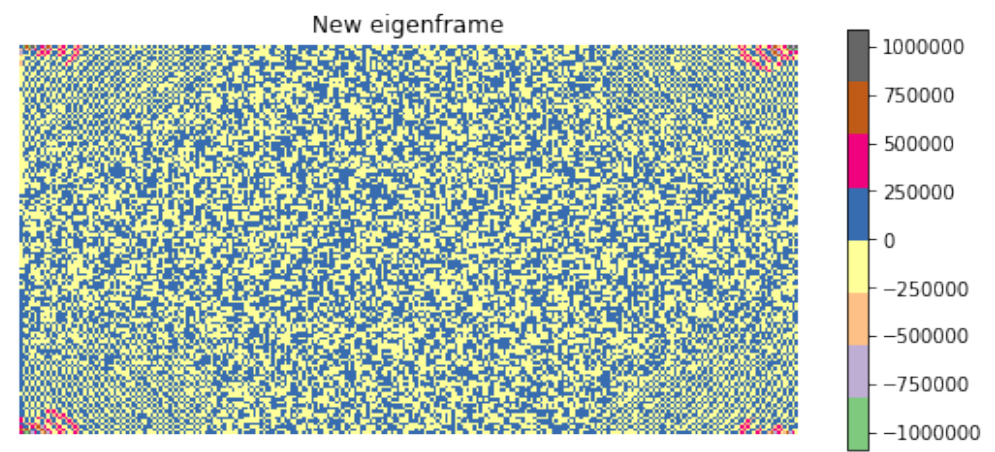

\section{Step 3: Correlate against eigenframe [Back to Section 0]}

Now we correlate against the eigenframe by translating the eigenframe in the frequency domain to the position of the frame. This provides a refinement of the position.

\subsection{Step 3.a: Shift the eigenframe to the translated frame [Back to Section 0]}

As before, we build shift arrays (notice a negative in the exponential this time).

[]: \# Build arrays to shift eigenframe to image against which we want to correlate

shift_in_z $=[]$

shift_in-y $=[]$

for $\mathrm{y}$ in range( $\mathrm{Ny})$

if $\mathrm{y}<\mathrm{Ny} / 2$ :

shift_in_y . append (np. exp $\left(-2 \cdot * n p \cdot p i * n p . \operatorname{complex}(0,1) * y_{-}\right.$translation* $\left.\left.(y / N y)\right)\right)$

shift_in_y. append (np. exp $\left.\left(-2, * n p . p i * n p . c o m p l e x(0,1) * y_{-} \operatorname{translation} *((\mathrm{y}-\mathrm{Ny}) / \mathrm{Ny})\right)\right)$

shift_in_y $=$ np. array (shift_in_y)

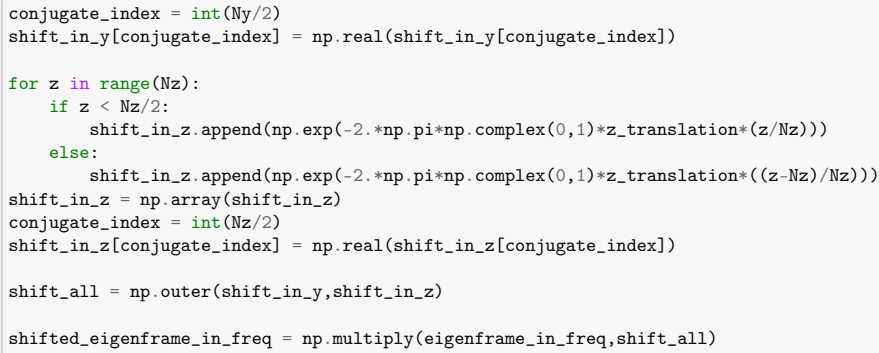

\subsection{Step 3.b: Compute phase correlation and locate maximum [Back to Section 0]}

The register_traslation() function takes, as the position estimate, the column with the highest cross-correlation value.

[]: \# Take shape for np. unrave

shape $=$ frame_in_freq. shape

\# Form cross-correlation

image_product $=$ frame_in_freq $*$ shifted_eigenframe_in_freq. $\operatorname{conj}()$

cross_correlation $=$ np.fft. ifftn(image_product)

\# Locate maximum

maxima $=$ np.unravel_index (np. $\operatorname{argmax}(n p \cdot a b s($ cross_correlation) $)$, cross_correlation. shape)

midpoints = np.array ([np.fix (axis_size / 2) for axis_size in shape])

\# Estimate peak location

shifts $=$ np.array (maxima, dtype=np.float64)

shifts [shifts > midpoints] -= np.array (shape) [shifts > midpoints]

\subsection{Step 3.c: Upsample to determine sub-pixel peak location [Back to Section 0]}

To upsample the cross-correlation peak, we take a 1.5 × 1.5 pixel box around the estimated peak location and build an interpolated grid with upsample_factor points in each dimension. See the documentation on_upsampled_dft.

[]: \# Initial shift estimate in upsampled grid

shifts $=$ np.round (shifts $*$ upsample_factor $) /$ upsample_factor 


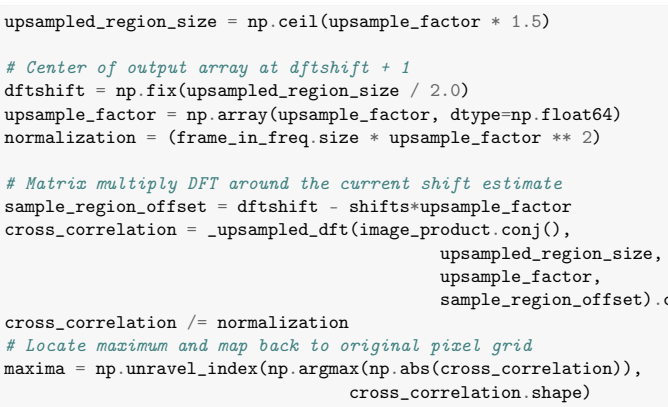

\subsection{Step 3.d: Error estimate with curve_fit() [Back to Section 0]}

The peak of the upsampled region is found the same way as the peak estimate (locating the column with the highest value). But we would like a good error estimate of this peak, so we fit a slice of the surface containing the highest value to a quadratic curve and report the error as given by curve_f $i t()$.

The quadratic function we define has a peak centered at $C$. The error that curve fit () returns is the covariance of parameters $A, B$, and $C$, which we use to find the one standard deviation error for $C$ (see the curve_fit () documentation). This is the error we report for the translation values.

[]: \# Define the quadratic function for fitting to the correlation surface

def tys_quad $(z, A, B, C)$ :

return $\mathrm{A} *(\mathbf{z}-\mathrm{C}) * * 2+\mathrm{B}$

\# Find error in peak by fitting a quadratic

\# Find length of arrays for curve_fit()

z_slice_length = np. shape (cross_correlation) $[1]$

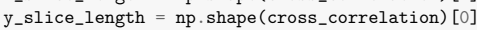

\# Set convenient domain for curve_fit()

$\mathrm{z}_{\text {_slice_domain }}=\mathrm{np} \cdot \mathrm{zeros}\left(\mathrm{z}_{\text {_slice_length}}\right)$

y_slice_domain $=$ np. zeros (y_slice_length)

for $\mathrm{k}$ in range ( $\mathrm{z}_{-}$slice_length):

$\mathrm{z}_{-}$slice_domain $[\mathrm{k}]=\mathrm{k}$

for $\mathrm{k}$ in range(y_slice_length):

y_slice_domain $[\mathrm{k}]=\mathrm{k}$

\# Pull range information from upsampled cross-correlation

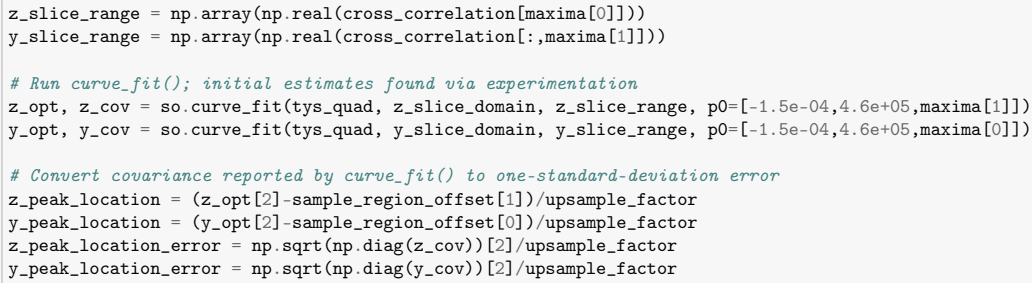

\subsection{Step 3.e: Refinement [Back to Section 0]}

Since we shifted the eigenframe to the microsphere location in the translated frame, our peak location is a refinement of the previous position found. We collate and report the new position, along with error. This is a one-standard-deviation error (see curve_fit () documentation).

[]: \# Combine peak estimate with upsampled peak location

maxima $=$ np. array (maxima, dtype=np.float64) - dftshift

shifts $=$ shifts + maxima upsample_factor

\# Print peak location with error estimate

print ("We estimate the peak in the z-direction to be at \% pixels" \% (z_translation+z peak_location))

print("The one-standard-devition error in z peak location is \% $\%$ \% z_peak_location_error)

print("We estimate the peak in the y-direction to be at \% pixels" \% (y_translation+y_peak_location))

print("The one-standard-devition error in y peak location is \% $\%$ \% y_peak_location_error)

\section{Step 4: Iterate for desired precision [Back to Section 0]}

Using the refined $z$ - and $y$-translations found in Step 3.e, we may repeat Steps 2 and 3: use the refined translation values to build another eigenframe and correlate against it. We iterated until we acheived a precision that was below that of the camera readout noise. Denote by $d_{n}\left(t_{i}\right)$ the $z$-translation of the microsphere at time $t_{i}$ when correlated against eigenframe $n(n=1,2, \ldots, 5)$. We computed the standard deviation of $d_{n}\left(t_{i}\right)-d_{n-1}\left(t_{i}\right)$ over all $t_{i}$ and found that the position differences quickly (after three iterations) reach a standard deviation below the physical resolution limit.

\section{Step 5: Output this notebook to LTEX-formatted PDF file [Back to Section 0]}


The following code cell converts this Jupyter notebook into a proper, clickable LATEX-formatted PDF file. After the cell is successfully run, the generated PDF may be found in the root Big $G$ data analysis directory, with filename Tutorial-BigG_Image_Analysis.pdf (Note that clicking on this link may not work; you may need to open the PDF file through another means.)

[]: import cmdline_helper as cmd \#NRPyt: Multi-platform Python command-line interface

cmd . output_Jupyter_notebook_to_LaTeXed_PDF("Tutorial-BigG_Image_Analysis") 


\section{References}

[1] C. W. Misner, K. S. Thorne, and J. A. Wheeler. Gravitation. W. H. Freeman, 1973.

[2] J. M. Weisberg and J. H. Taylor. The relativistic binary pulsar b1913+16: Thirty years of observations and analysis. Binary Radio Pulsars, 328:25-31, 2005.

[3] Spiral dance of black holes. https://www.ligo.caltech.edu/image/ligo20160615f. Accessed: 2020-03-09.

[4] C. T. Bolton. Identification of Cygnus X-1 with HDE 226868. Nature, 235(5336):271273, February 1972.

[5] J. M. Weisberg, J. H. Taylor, and L. A. Fowler. Gravitational waves from an orbiting pulsar. Scientific American, 245:74-82, October 1981.

[6] File:ligo schematic (multilang).svg. https://en.wikipedia.org/wiki/File:LIGO_ schematic_(multilang).svg. Accessed: 2020-03-03.

[7] J. Veitch, V. Raymond, B. Farr, W. Farr, P. Graff, S. Vitale, B. Aylott, K. Blackburn, N. Christensen, M. Coughlin, W. Del Pozzo, F. Feroz, J. Gair, C.-J. Haster, V. Kalogera, T. Littenberg, I. Mandel, R. O'Shaughnessy, M. Pitkin, C. Rodriguez, C. Röver, T. Sidery, R. Smith, M. Van Der Sluys, A. Vecchio, W. Vousden, and L. Wade. Parameter estimation for compact binaries with ground-based gravitational-wave observations using the LALInference software library. Phys. Rev. D, 91(4):042003:1-25, February 2015.

[8] A. Buonanno and T. Damour. Effective one-body approach to general relativistic twobody dynamics. Phys. Rev. D, 59(8):084006:1-24, April 1999. 
[9] A. Buonanno, Y. Chen, and T. Damour. Transition from inspiral to plunge in precessing binaries of spinning black holes. Phys. Rev. D, 74(10):104005:1-26, November 2006.

[10] Sean T. McWilliams. Analytical black-hole binary merger waveforms. Phys. Rev. Lett., 122:191102, May 2019.

[11] NSF Ideas Laboratory at NIST: Measuring "Big G" Challenge. https://tinyurl.com/ MeasBigG. Accessed: 2020-03-03.

[12] Henry Cavendish. Xxi. experiments to determine the density of the earth. Philos. Trans. Royal Soc., 88:469-526, 1798.

[13] S. van der Walt, J. L. Schönberger, J. Nunez-Iglesias, F. Boulogne, J. D. Warner, N. Yager, E. Gouillart, T. Yu, and the scikit-image contributors. scikit-image: Image processing in Python. PeerJ, 2:e453, 2014.

[14] T. D. Knowles, C. Devine, D. A. Buch, S. A. Bilgili, T. R. Adams, Z. B. Etienne, and S. T. McWilliams. Improving performance of SEOBNRv3 by $\sim 300 \mathrm{x}$. Classical and Quantum Gravity, 35(15):155003, June 2018.

[15] B. P. Abbott, R. Abbott, T. D. Abbott, M. R. Abernathy, F. Acernese, K. Ackley, C. Adams, T. Adams, P. Addesso, R. X. Adhikari, et al. Observation of Gravitational Waves from a Binary Black Hole Merger. Phys. Rev. Lett., 116(6):061102:1-16, February 2016.

[16] B. P. Abbott, R. Abbott, T. D. Abbott, M. R. Abernathy, F. Acernese, K. Ackley, C. Adams, T. Adams, P. Addesso, R. X. Adhikari, et al. GW151226: Observation of Gravitational Waves from a 22-Solar-Mass Binary Black Hole Coalescence. Phys. Rev. Lett., 116(24):241103:1-14, June 2016.

[17] B. P. Abbott, R. Abbott, T. D. Abbott, F. Acernese, K. Ackley, C. Adams, T. Adams, P. Addesso, R. X. Adhikari, V. B. Adya, and et al. GW170104: Observation of a 50-Solar-Mass Binary Black Hole Coalescence at Redshift 0.2. Phys. Rev. Lett., 118(22):221101:1-17, June 2017. 
[18] The LIGO Scientific Collaboration, the Virgo Collaboration, B. P. Abbott, R. Abbott, T. D. Abbott, F. Acernese, K. Ackley, C. Adams, T. Adams, P. Addesso, and et al. GW170608: Observation of a 19-solar-mass Binary Black Hole Coalescence. ArXiv e-prints, November 2017.

[19] B. P. Abbott, R. Abbott, T. D. Abbott, F. Acernese, K. Ackley, C. Adams, T. Adams, P. Addesso, R. X. Adhikari, V. B. Adya, and et al. GW170814: A Three-Detector Observation of Gravitational Waves from a Binary Black Hole Coalescence. Physical Review Letters, 119(14):141101, October 2017.

[20] B. P. Abbott, R. Abbott, T. D. Abbott, F. Acernese, K. Ackley, C. Adams, T. Adams, P. Addesso, R. X. Adhikari, V. B. Adya, and et al. GW170817: Observation of Gravitational Waves from a Binary Neutron Star Inspiral. Physical Review Letters, 119(16):161101, October 2017.

[21] The LIGO Scientific Collaboration. LALSuite: LSC Algorithm Library Suite, 2016. https://www.lsc-group.phys.uwm.edu/daswg/projects/lalsuite.html.

[22] Y. Pan, A. Buonanno, A. Taracchini, L. E. Kidder, A.H. Mroué, H. P. Pfeiffer, M. A. Scheel, and B. Szilágyi. Inspiral-merger-ringdown waveforms of spinning, precessing black-hole binaries in the effective-one-body formalism. Phys. Rev. D, 89(8):084006:118, April 2014.

[23] L. Santamaría, F. Ohme, P. Ajith, B. Brügmann, N. Dorband, M. Hannam, S. Husa, P. Mösta, D. Pollney, C. Reisswig, E. L. Robinson, J. Seiler, and B. Krishnan. Matching post-Newtonian and numerical relativity waveforms: Systematic errors and a new phenomenological model for nonprecessing black hole binaries. Phys. Rev. D, 82(6):064016:1-21, September 2010.

[24] M. Hannam, P. Schmidt, A. Bohé, L. Haegel, S. Husa, F. Ohme, G. Pratten, and M. Pürrer. Simple Model of Complete Precessing Black-Hole-Binary Gravitational Waveforms. Phys. Rev. Lett., 113(15):151101:1-5, October 2014. 
[25] S. Husa, S. Khan, M. Hannam, M. Pürrer, F. Ohme, X. Jiménez Forteza, and A. Bohé. Frequency-domain gravitational waves from nonprecessing black-hole binaries. I. New numerical waveforms and anatomy of the signal. Phys. Rev. D, 93(4):044006:1-19, February 2016.

[26] S. Khan, S. Husa, M. Hannam, F. Ohme, M. Pürrer, X. Jiménez Forteza, and A. Bohé. Frequency-domain gravitational waves from nonprecessing black-hole binaries. II. A phenomenological model for the advanced detector era. Phys. Rev. D, 93(4):044007:127, February 2016.

[27] S. Babak, A. Taracchini, and A. Buonanno. Validating the effective-one-body model of spinning, precessing binary black holes against numerical relativity. Phys. Rev. D, 95(2):024010, January 2017.

[28] M. Pürrer. Frequency domain reduced order model of aligned-spin effective-one-body waveforms with generic mass ratios and spins. Phys. Rev. D, 93(6):064041:1-15, March 2016.

[29] S. E. Field, C. R. Galley, J. S. Hesthaven, J. Kaye, and M. Tiglio. Fast prediction and evaluation of gravitational waveforms using surrogate models. Phys. Rev. X, 4(3):031006:1-21, July 2014.

[30] S. E. Field, C. R. Galley, and E. Ochsner. Towards beating the curse of dimensionality for gravitational waves using reduced basis. Phys. Rev. D, 86(8):084046:1-7, October 2012.

[31] C. Devine, Z. B. Etienne, and S. T. McWilliams. Optimizing spinning time-domain gravitational waveforms for advanced LIGO data analysis. Class. Quantum Grav., 33(12):125025:1-15, June 2016.

[32] A. Taracchini, A. Buonanno, Y. Pan, T. Hinderer, M. Boyle, D. A. Hemberger, L. E. Kidder, G. Lovelace, A. H. Mroué, H. P. Pfeiffer, M. A. Scheel, B. Szilágyi, N. W. Taylor, and A. Zenginoglu. Effective-one-body model for black-hole binaries with generic mass ratios and spins. Phys. Rev. D, 89(6):061502:1-6, March 2014. 
[33] R. M. Stallman and GCC DeveloperCommunity. Using The Gnu Compiler Collection. 51 Franklin Street, Fifth Floor, Boston, MA 02110-1301 USA, 2015.

[34] Intel. User and Reference Guide for the Intel C++ Compiler 15.0, 2014. https: //software.intel.com/en-us/compiler_15.0_ug_c.

[35] Wolfram Research, Inc. Mathematica 10.4. Champaign, Illinois, 2016. https://www. wolfram.com.

[36] N. Limare. Floating-Point Math Speed vs Precision, 2014. http://nicolas.limare. net/pro/notes/2014/12/16_math_speed/.

[37] W. H. Press, S. A. Teukolsky, W. T. Vetterling, and B. P. Flannery. Numerical Recipies: The Art of Scientific Computing - Third Edition. Cambridge University Press, 3rd edition, 2007. http://numerical.recipies.

[38] A. Nitz, I. Harry, C. M. Biwer, D. Brown, J. Willis, T. Dal Canton, L. Pekowsky, T. Dent, A. R. Williamson, C. Capano, et al. ligo-cbc/pycbc: O2 Production Release 11, April 2017. https://doi.org/10.5281/zenodo.556097.

[39] T. Dal Canton, A. H. Nitz, A. P. Lundgren, A. B. Nielsen, D. A. Brown, T. Dent, I. W. Harry, B. Krishnan, A. J. Miller, K. Wette, K. Wiesner, and J. L. Willis. Implementing a search for aligned-spin neutron star-black hole systems with advanced ground based gravitational wave detectors. Phys. Rev. D, 90(8):082004:1-17, October 2014.

[40] S. A. Usman, A. H. Nitz, I. W. Harry, C. M. Biwer, D. A. Brown, M. Cabero, C. D. Capano, T. Dal Canton, T. Dent, S. Fairhurst, M. S. Kehl, D. Keppel, B. Krishnan, A. Lenon, A. Lundgren, A. B. Nielsen, L. P. Pekowsky, H. P. Pfeiffer, P. R. Saulson, M. West, and J. L. Willis. The PyCBC search for gravitational waves from compact binary coalescence. Class. Quantum Grav., 33(21):215004:1-25, November 2016.

[41] A. Bohé, L. Shao, A. Taracchini, A. Buonanno, S. Babak, I. W. Harry, I. Hinder, S. Ossokine, M. Pürrer, V. Raymond, T. Chu, H. Fong, P. Kumar, H. P. Pfeiffer, M. Boyle, D. A. Hemberger, L. E. Kidder, G. Lovelace, M. A. Scheel, and B. Szilágyi. Improved 
effective-one-body model of spinning, nonprecessing binary black holes for the era of gravitational-wave astrophysics with advanced detectors. Phys. Rev. D, 95(4):044028:129, February 2017.

[42] LSC. Advanced LIGO anticipated sensitivity curves, 2010. https://dcc.ligo.org/ LIGO-T0900288/public.

[43] Kent Yagi and Nicolás Yunes. Love number can be hard to measure. Phys. Rev. D, 89:021303, Jan 2014.

[44] Black hole spins. https://bhdynamics.com/2018/01/02/black-hole-spins/. Accessed: 2020-03-30.

[45] E. Barausse and A. Buonanno. Improved effective-one-body hamiltonian for spinning black-hole binaries. Phys. Rev. D, 81:084024, April 2010.

[46] Project jupyter. https://jupyter.org/about. Accessed: 2020-03-10.

[47] A. Meurer, C. P. Smith, M. Paprocki, O. Čertík, S. B. Kirpichev, M. Rocklin, A. Kumar, S. Ivanov, J. K. Moore, S. Singh, T. Rathnayake, S. Vig, B. E. Granger, R. P. Muller, F. Bonazzi, H. Gupta, S. Vats, F. Johansson, F. Pedregosa, M. J. Curry, A. R. Terrel, Š. Roučka, A. Saboo, I. Fernando, S. Kulal, R. Cimrman, and Anthony Scopatz. Sympy: symbolic computing in python. PeerJ Computer Science, 3:e103, January 2017.

[48] Z. B. Etienne. Personal communication to T. D. Knowles. Received: 2019-05-14.

[49] Enrico Barausse and Alessandra Buonanno. Extending the effective-one-body hamiltonian of black-hole binaries to include next-to-next-to-leading spin-orbit couplings. Phys. Rev. D, 84:104027, November 2011.

[50] C. W. Lewandowski, T. D. Knowles, Z. B. Etienne, and Brian D’Urso. High sensitivity accelerometry with a feedback-cooled magnetically levitated microsphere. arXiv preprint:2002.07585, March 2020.

[51] T. M. Niebauer, G. S. Sasagawa, J. E. Faller, R. Hilt, and F. Klopping. A new generation of absolute gravimeters. Metrologia, 32(3):159, 1995. 
[52] Y. Bidel, O. Carraz, R. Charriere, M. Cadoret, N. Zahzam, and A. Bresson. Compact cold atom gravimeter for field applications. Appl. Phys. Lett., 102(14):144107, April 2013.

[53] J. Liu and K-D. Zhu. Nanogravity gradiometer based on a sharp optical nonlinearity in a levitated particle optomechanical system. Phys. Rev. D, 95(4):044014, February 2017.

[54] B. Battelier, B. Barrett, L. Fouché, L. Chichet, L. Antoni-Micollier, H. Porte, F. Napolitano, J. Lautier, A. Landragin, and P. Bouyer. Development of compact cold-atom sensors for inertial navigation. In Quantum Optics, volume 9900, page 990004. International Society for Optics and Photonics, April 2016.

[55] D. Kafri, J. M. Taylor, and G. J. Milburn. A classical channel model for gravitational decoherence. New J. Phys., 16(6):065020, June 2014.

[56] A. Albrecht, A. Retzker, and M. B. Plenio. Testing quantum gravity by nanodiamond interferometry with nitrogen-vacancy centers. Phys. Rev. A, 90(3):033834, September 2014.

[57] D. C. Moore. Tests of fundamental physics with optically levitated microspheres in high vacuum. In Optical Trapping and Optical Micromanipulation XV, volume 10723, page 107230H. International Society for Optics and Photonics, September 2018.

[58] O. Gerberding, F. Guzmán Cervantes, J. Melcher, J. R. Pratt, and J. M. Taylor. Optomechanical reference accelerometer. Metrologia, 52(5):654, April 2015.

[59] Y. Bao, F. Guzmán Cervantes, A. Balijepalli, J. R. Lawall, J. M. Taylor, T. W. LeBrun, and J. J. Gorman. An optomechanical accelerometer with a high-finesse hemispherical optical cavity. In 2016 IEEE International Symposium on Inertial Sensors and Systems, pages 105-108. IEEE, February 2016.

[60] F. Guzmán Cervantes, L. Kumanchik, J. Pratt, and J. M. Taylor. High sensitivity optomechanical reference accelerometer over 10 khz. Appl. Phys. Lett., 104(22):221111, June 2014. 
[61] J. Moser, A. Eichler, J. Güttinger, M. I. Dykman, and A. Bachtold. Nanotube mechanical resonators with quality factors of up to 5 million. Nat. Nanotechnol., 9(12):1007, October 2014.

[62] R. A. Norte, J. P. Moura, and S. Gröblacher. Mechanical resonators for quantum optomechanics experiments at room temperature. Phys. Rev. Lett., 116(14):147202, April 2016.

[63] A. G. Krause, M. Winger, T. D. Blasius, Q. Lin, and O. Painter. A high-resolution microchip optomechanical accelerometer. Nat. Photonics, 6(11):768, October 2012.

[64] Y. L. Li and P. F. Barker. Characterization and testing of a micro-g whispering gallery mode optomechanical accelerometer. J. Light. Technol., 36(18):3919-3926, July 2018.

[65] M. Armano, H. Audley, G. Auger, J. T. Baird, M. Bassan, P. Binetruy, M. Born, D. Bortoluzzi, N. Brandt, M. Caleno, and et al. Sub-femto-g free fall for space-based gravitational wave observatories: Lisa pathfinder results. Phys. Rev. Lett., 116(23):231101, June 2016.

[66] N. Yu, J. M. Kohel, J. R. Kellogg, and L. Maleki. Development of an atom-interferometer gravity gradiometer for gravity measurement from space. Appl. Phys. B, 84(4):647-652, September 2006.

[67] G. Stern, B. Battelier, R. Geiger, G. Varoquaux, A. Villing, F. Moron, O. Carraz, N. Zahzam, Y. Bidel, W. Chaibi, and et al. Light-pulse atom interferometry in microgravity. Eur. Phys. J. D, 53(3):353-357, May 2009.

[68] G. Biedermann. Gravity tests, differential accelerometry and interleaved clocks with cold atom interferometers. Stanford University, 2008.

[69] M-K. Zhou, Z-K. Hu, X-C. Duan, B-L. Sun, L-L. Chen, Q-Z. Zhang, and J. Luo. Performance of a cold-atom gravimeter with an active vibration isolator. Phys. Rev. A, 86(4):043630, October 2012. 
[70] G. W. Biedermann, X. Wu, L. Deslauriers, S. Roy, C. Mahadeswaraswamy, and M. A. Kasevich. Testing gravity with cold-atom interferometers. Phys. Rev. A, 91(3):033629, December 2014.

[71] G. Ranjit, D. P. Atherton, J. H. Stutz, M. Cunningham, and A. A. Geraci. Attonewton force detection using microspheres in a dual-beam optical trap in high vacuum. Phys. Rev. A, 91(5):051805, March 2015.

[72] G. Ranjit, M. Cunningham, K. Casey, and A. A. Geraci. Zeptonewton force sensing with nanospheres in an optical lattice. Phys. Rev. A, 93(5):053801, May 2016.

[73] C. Timberlake, G. Gasbarri, A. Vinante, A. Setter, and H. Ulbricht. Acceleration sensing with magnetically levitated oscillators above a superconductor. Appl. Phys. Lett., 115(22):224101, November 2019.

[74] A. Ashkin and J. M. Dziedzic. Optical levitation by radiation pressure. Appl. Phys. Lett., 19(8):283-285, 1971.

[75] F. Monteiro, S. Ghosh, A. G. Fine, and D. C. Moore. Optical levitation of 10-ng spheres with nano-g acceleration sensitivity. Phys. Rev. A, 96(6):063841, December 2017.

[76] A. D. Rider, C. P. Blakemore, G. Gratta, and D. C. Moore. Single-beam dielectricmicrosphere trapping with optical heterodyne detection. Phys. Rev. A, 97(1):013842, October 2017.

[77] M. C. O’Brien, S. Dunn, J. E. Downes, and J. Twamley. Magneto-mechanical trapping of micro-diamonds at low pressures. Appl. Phys. Lett., 114(5):053103, February 2019.

[78] J. P. Houlton, M. L. Chen, M. D. Brubaker, K. A. Bertness, and C. T. Rogers. Axisymmetric scalable magneto-gravitational trap for diamagnetic particle levitation. Rev. Sci. Instrum., 89(12):125107, December 2018.

[79] J-F. Hsu, P. Ji, C. W. Lewandowski, and B. D'Urso. Cooling the motion of diamond nanocrystals in a magneto-gravitational trap in high vacuum. Sci. Rep., 6:30125, July 2016. 
[80] W. M. Klahold, C. W. Lewandowski, P. Nachman, B. R. Slezak, and B. D’Urso. Precision optomechanics with a particle in a magneto-gravitational trap. In Optical, OptoAtomic, and Entanglement-Enhanced Precision Metrology, volume 10934, page 109340P. International Society for Optics and Photonics, 2019.

[81] F. Monteiro, W. Li, G. Afek, C-l. Li, M. Mossman, and D. C. Moore. Force and acceleration sensing with optically levitated nanogram masses at microkelvin temperatures. arXiv preprint:2001.10931, 2020.

[82] U. Delić, M. Reisenbauer, K. Dare, D. Grass, V. Vuletić, N. Kiesel, and M. Aspelmeyer. Cooling of a levitated nanoparticle to the motional quantum ground state. Science, February 2020.

[83] C. W. Lewandowski, W. R. Babbitt, and B. D'Urso. Comparison of magnetogravitational and optical trapping for levitated optomechanics. In Optical Trapping and Optical Micromanipulation XVI, volume 11083, page 110831C. International Society for Optics and Photonics, September 2019.

[84] M. D. Simon and A. K. Geim. Diamagnetic levitation: flying frogs and floating magnets. J. Appl. Phys., 87(9):6200-6204, April 2000.

[85] B. R. Slezak, C. W. Lewandowski, J-F. Hsu, and B. D'Urso. Cooling the motion of a silica microsphere in a magneto-gravitational trap in ultra-high vacuum. New J. Phys., 20(6):063028, June 2018.

[86] R. R. Perron. The design and application of a reliable ultrasonic atomizer. IEEE Transactions on Sonics and Ultrasonics, 14(4):149-152, October 1967.

[87] W. Paul. Electromagnetic traps for charged and neutral particles. Rev. Mod. Phys., 62(3):531, July 1990.

[88] D. J. Douglas, A. J. Frank, and D. Mao. Linear ion traps in mass spectrometry. Mass Spectrom. Rev., 24(1):1-29, April 2004.

[89] Texas Instruments. Stacking the REF50xx for high voltage references, May 2013. 
[90] J M W Milatz, J J Van Zolingen, and BB Van Iperen. The reduction in the brownian motion of electrometers. Physica, 19(1-12):195-202, 1953.

[91] M. Guizar-Sicairos, S. T. Thurman, and Ja. R. Fienup. Efficient subpixel image registration algorithms. Opt. Lett., 33(2):156-158, January 2008.

[92] K. Y. Yasumura, T. D. Stowe, E. M. Chow, T. Pfafman, T. W. Kenny, B. C. Stipe, and D. Rugar. Quality factors in micron-and submicron-thick cantilevers. J. Microelectromech. Syst., 9(1):117-125, March 2000.

[93] A. A. Geraci, S. B. Papp, and J. Kitching. Short-range force detection using optically cooled levitated microspheres. Phys. Rev. Lett., 105(10):101101, September 2010.

[94] Sercel Inc. Geophones: Seismic Sensors, December 2019.

[95] R. Kirchhoff, C. M. Mow-Lowry, V. B. Adya, G. Bergmann, S. Cooper, M. M. Hanke, P. Koch, S. M. Köhlenbeck, J. Lehmann, P. Oppermann, and et al. Huddle test measurement of a near johnson noise limited geophone. Rev. Sci. Instrum., 88(11):115008, November 2017.

[96] Texas Instruments Inc. OPA188 Precision, Low-Noise, Rail-to-Rail Output, 36-V, ZeroDrift Operational Amplifier, September 2016. 2013-Revised 2016.

[97] C. W. Lewandowski, T. D. Knowles, Z. B. Etienne, and B. D'Urso. Active optical table tilt stabilization. arXiv preprint:2003.03404, 2020.

[98] J. D. Teufel, T. Donner, D. Li, J. W. Harlow, M. S. Allman, K. Cicak, A. J. Sirois, J. D. Whittaker, K. W. Lehnert, and R. W. Simmonds. Sideband cooling of micromechanical motion to the quantum ground state. Nature, 475(7356):359-363, July 2011. 